\title{
Tren-based tris-macrocycles as anion hosts. Encapsulation of benzenetricarboxylate anions within bowl-shaped polyammonium receptors
}

Carla Bazzicalupi, Antonio Bianchi, Andrea Bencini, Lucia Borsari, Claudia Giorgi, Barbara Valtancoli, Carmen Anda, Antoni Llobet

\section{SUPPORTING INFORMATION}

Figure S1. ORTEP drawings of the crystal structure of $\left[\left(\mathrm{Na}\left(\mathrm{ClO}_{4}\right)_{6}\right) \subset \mathbf{L} \mathbf{1}_{2} \mathrm{H}_{13}\right]^{8+}$

Page S4

Figure S2. $\mathrm{pH}$ dependence of the ${ }^{1} \mathrm{H}$ NMR signals of $\mathbf{L} 2$ and distribution diagram of the $\mathbf{L 2}$ protonated species Page S5

Figure S3. Distribution diagrams of the complexes of $\mathbf{L 1}$ with 1,2,3-BTC and 1,2,4 BTC.

Page S6

Figure S4. Distribution diagrams of the complexes of $\mathbf{L 2}$ with 1,2,3-BTC and 1,2,4 BTC.

Page S7

Figure S5. Plots of the chemical shifts $\left(\delta\right.$, ppm) of the ${ }^{1} \mathrm{H}$ NMR signals of 1,3,5-BTC, 1,2,4-BTC and 1,2,3-

BTC in the presence of increasing amount of $\mathbf{L 1}$.

Page S8

Figure S6. Plots of the chemical shifts ( $\delta$, ppm) of the ${ }^{1} \mathrm{H}$ NMR signals of 1,3,5-BTC, 1,2,4-BTC and 1,2,3-

BTC in the presence of increasing amount of $\mathbf{L 2}$.

Page S9

Table S1. Energy content of the lowest energy conformers of the $\left[\mathrm{H}_{n} \mathbf{L}\right]^{\mathrm{n}+}$ free receptors, $\mathrm{BTC}^{3-}$ free anions and $\left[\mathrm{H}_{\mathrm{n}} \mathbf{L}(\mathrm{A})\right]^{(\mathrm{n}-3)+}$ adducts.

Table S2. Cartesian coordinates, atomic charges and atom types for the lowest energy conformer of the $\left[\mathrm{H}_{7} \mathbf{L 1}\right]^{7+}$ protonated receptor.

Table S3. Cartesian coordinates, atomic charges and atom types for the lowest energy conformer of the $\left[\mathrm{H}_{9} \mathbf{L 2}\right]^{9+}$ protonated receptor.

Table S4. Cartesian coordinates, atomic charges and atom types for the lowest energy conformer of the 1,3,5BTC trianion.

Table S5. Cartesian coordinates, atomic charges and atom types for the lowest energy conformer of the 1,2,4BTC trianion.

Table S6. Cartesian coordinates, atomic charges and atom types for the lowest energy conformer of the 1,2,3BTC trianion.

Description of the lowest energy conformer of the family with endo conformation of the N1 bridgehead nitrogen for the $\left[\mathrm{H}_{7} \mathbf{L} \mathbf{1 A}\right]^{4+}$ adduct $(\mathrm{A}=1,3,5-\mathrm{BTC})$

Table S7. Hydrogen bond interactions for the lowest energy conformer of the families with exo and endo conformation of the $\mathrm{N} 1$ bridgehead nitrogen for the $\left[\mathrm{H}_{7} \mathbf{L} \mathbf{1} \mathrm{A}\right]^{4+}$ adduct $(\mathrm{A}=1,3,5-\mathrm{BTC})$.

Figure S7. Lowest energy conformers of the families with exo and endo conformation of the N1 bridgehead nitrogen for the $\left[\mathrm{H}_{7} \mathbf{L} \mathbf{1} \mathrm{A}\right]^{4+}$ adduct $(\mathrm{A}=1,3,5-\mathrm{BTC})$. 
Table S8. Cartesian coordinates for the lowest energy conformer of the complex $\left[\mathrm{H}_{7} \mathbf{L} \mathbf{1 A}\right]^{4+}(\mathrm{A}=1,3,5-\mathrm{BTC})$ with exo conformation of the bridgehead N1 nitrogen (a)

Table S9. Cartesian coordinates for the lowest energy conformer of the complex $\left[\mathrm{H}_{7} \mathbf{L} \mathbf{1} A\right]^{4+}(\mathrm{A}=1,3,5-\mathrm{BTC})$ with endo conformation of the bridgehead N1 nitrogen ....

Description of the lowest energy conformer of the family with endo conformation of the N1 bridgehead nitrogen for the $\left[\mathrm{H}_{7} \mathbf{L} \mathbf{1 A}\right]^{4+}$ adduct $(\mathrm{A}=1,2,4-\mathrm{BTC})$.

Table S10. Hydrogen bond interactions for the lowest energy conformer of the families with exo and endo conformation of the $\mathrm{N} 1$ bridgehead nitrogen for the $\left[\mathrm{H}_{7} \mathbf{L 1} \mathrm{A}\right]^{4+}$ adduct $(\mathrm{A}=1,2,4-\mathrm{BTC})$.

Figure S8. Lowest energy conformers of the families with exo and endo conformation of the N1 bridgehead nitrogen for the $\left[\mathrm{H}_{7} \mathbf{L 1} \mathrm{A}\right]^{4+}$ adduct $(\mathrm{A}=1,2,4-\mathrm{BTC})$...

Table S11. Cartesian coordinates for the lowest energy conformer of the complex $\left[\mathrm{H}_{7} \mathbf{L} \mathbf{A}\right]^{4+}(\mathrm{A}=1,2,4-\mathrm{BTC})$ with exo conformation of the bridgehead N1 nitrogen

Table S12. Cartesian coordinates for the lowest energy conformer of the complex $\left[\mathrm{H}_{7} \mathbf{L} \mathbf{1} \mathrm{A}\right]^{4+}(\mathrm{A}=1,2,4-\mathrm{BTC})$ with endo conformation of the bridgehead N1 nitrogen

Description of the lowest energy conformer of the family with exo conformation of the $\mathrm{N} 1$ bridgehead nitrogen for the $\left[\mathrm{H}_{7} \mathbf{L} \mathbf{1 A}\right]^{4+}$ adduct $(\mathrm{A}=1,2,3-\mathrm{BTC})$.

Page S36

Table S13. Hydrogen bond interactions for the lowest energy conformer of the families with endo and exo conformation of the $\mathrm{N} 1$ bridgehead nitrogen for the $\left[\mathrm{H}_{7} \mathbf{L} \mathbf{1} \mathrm{A}\right]^{4+}$ adduct $(\mathrm{A}=1,2,3-\mathrm{BTC})$.

Figure S9. Lowest energy conformers of the families with endo and exo conformation of the N1 bridgehead nitrogen for the $\left[\mathrm{H}_{7} \mathbf{L 1} \mathrm{A}\right]^{4+}$ adduct $(\mathrm{A}=1,2,3-\mathrm{BTC})$

Table S14. Cartesian coordinates for the lowest energy conformer of the complex $\left[\mathrm{H}_{7} \mathbf{L 1} \mathrm{A}\right]^{4+}(\mathrm{A}=1,2,3-\mathrm{BTC})$ with endo conformation of the bridgehead N1 nitrogen.

Table S15. Cartesian coordinates for the lowest energy conformer of the complex $\left[\mathrm{H}_{7} \mathbf{L 1 A}\right]^{4+}(\mathrm{A}=1,2,3-\mathrm{BTC})$ with exo conformation of the bridgehead N1 nitrogen

Page S41

Description of the lowest energy conformer for the $\left[\mathrm{H}_{9} \mathbf{L} 2 \mathrm{~A}\right]^{6+}$ adducts $(\mathrm{A}=1,3,5$-BTC , 1,2,3-BTC and 1,2,4BTC)

Table S16. Hydrogen bond interactions for the lowest energy conformer of the $\left[\mathrm{H}_{9} \mathbf{L} 2 \mathrm{~A}\right]^{6+}$ adduct $(\mathrm{A}=1,3,5-$ BTC)

Figure S10. Lowest energy conformer of the $\left[\mathrm{H}_{9} \mathbf{L} 2 \mathrm{~A}\right]^{6+}$ adduct $(\mathrm{A}=1,3,5-\mathrm{BTC}) \ldots$

Table S17. Cartesian coordinates for the lowest energy conformer of the complex $\left[\mathrm{H}_{9} \mathbf{L} \mathbf{2} \mathrm{A}\right]^{6+}(\mathrm{A}=1,3,5-\mathrm{BTC})$

Table S18. Hydrogen bond interactions for the lowest energy conformer of the $\left[\mathrm{H}_{9} \mathbf{L} 2 \mathrm{~A}\right]^{6+}$ adduct $(\mathrm{A}=1,2,4-$ BTC).

Figure S11. Lowest energy conformer of the $\left[\mathrm{H}_{9} \mathbf{L} 2 \mathrm{~A}\right]^{6+}$ adduct $(\mathrm{A}=1,2,4-\mathrm{BTC})$

Table S19. Cartesian coordinates for the lowest energy conformer of the complex $\left[\mathrm{H}_{9} \mathbf{L} \mathbf{2} \mathrm{A}\right]^{6+}(\mathrm{A}=1,2,4-\mathrm{BTC})$

Table S20. Hydrogen bond interactions for the lowest energy conformer of the $\left[\mathrm{H}_{9} \mathbf{L} 2 \mathrm{~A}\right]^{6+}$ adduct $(\mathrm{A}=1,2,3-$ BTC)

Figure S12. Lowest energy conformer of the $\left[\mathrm{H}_{9} \mathbf{L 2} \mathrm{A}\right]^{6+}$ adduct $(\mathrm{A}=1,2,3-\mathrm{BTC})$. 
Table S21. Cartesian coordinates for the lowest energy conformer of the complex $\left[\mathrm{H}_{9} \mathbf{L} \mathbf{2 A}\right]^{6+}(\mathrm{A}=1,2,3-\mathrm{BTC})$ Page S55

Table S22. Thermodynamics parameters for $\mathbf{L} 1$ and $\mathbf{L} 2$ protonation. Page S58

Table S23. Thermodynamics parameters for protonation of the BTC acids

Figure S13. pH dependence of the enthalpic and entropic conditional contributions for the complexation of 1,3,5-BTC, 1,2,4-BTC and 1,2,3-BTC with L2

Page S59 

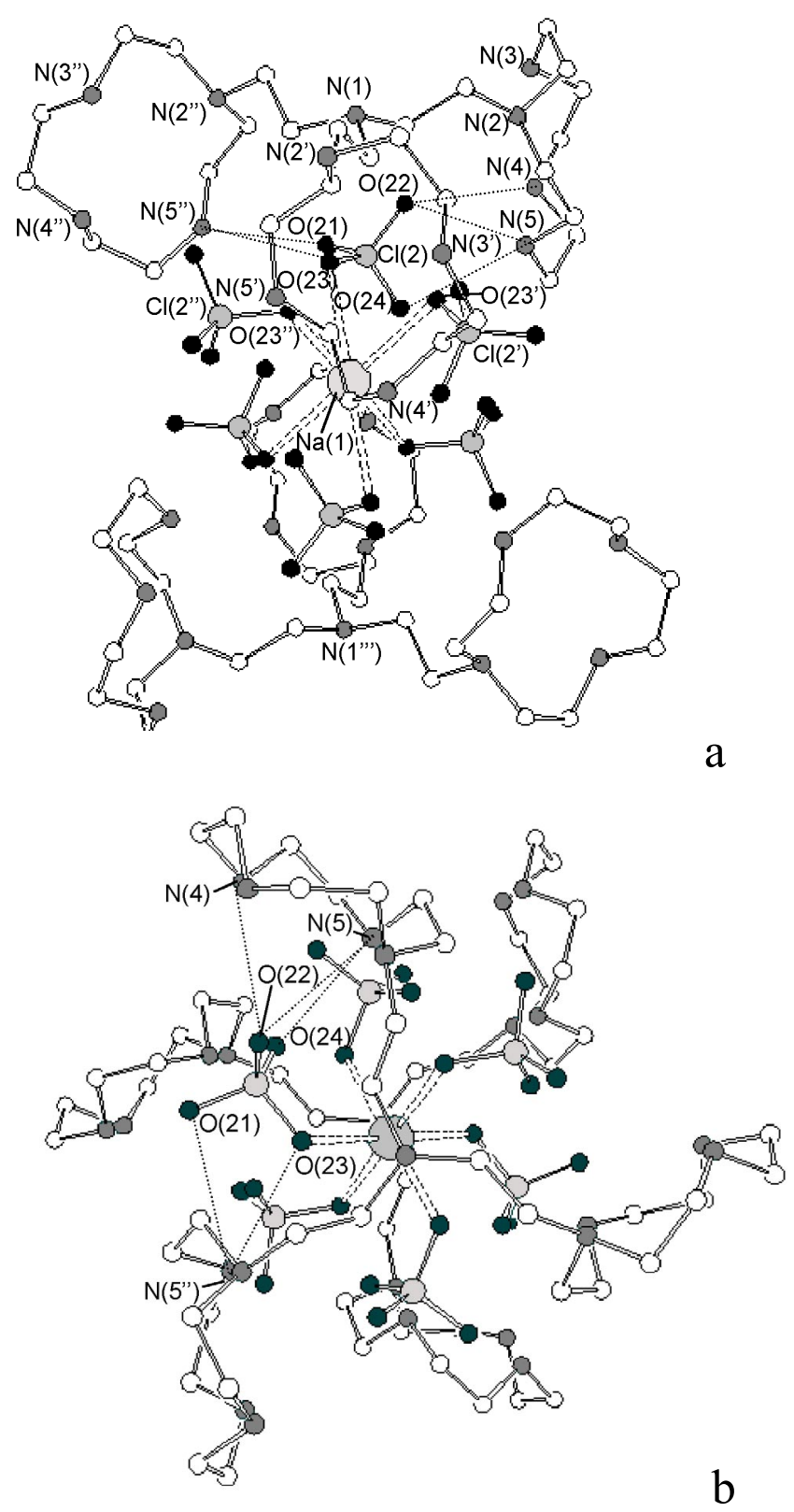

Figure S1. ORTEP drawings (a: side view, b: top view) of the crystal structure of $\left[\left(\mathrm{Na}\left(\mathrm{ClO}_{4}\right)_{6}\right) \subset \mathbf{L} 1_{2} \mathrm{H}_{13}\right]^{8+}$, taken from reference 26 (Bazzicalupi C.; Bencini A.; Berni, E.; Bianchi, A.; Ciattini, S.; Giorgi, C.; Maoggi, S.; Paoletti P.; Valtancoli B. J. Org. Chem., 2002, 67, 9107-9110). Hydrogen bond interactions involving the $\mathrm{Cl}(2)$ perchlorate: $\mathrm{N}(5) \cdots \mathrm{O}(22)$,

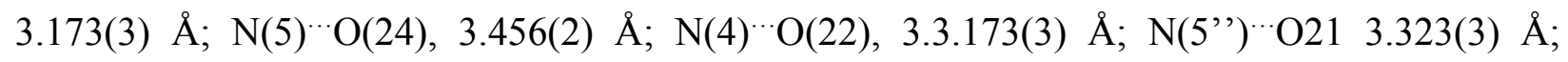
$\mathrm{N}\left(5^{\prime \prime}\right) \cdots \mathrm{O}(22), 2.932(2) \AA$. The hydrogen bond interactions involving the other symmetry related five perchlorate anions are not shown for clarity. 


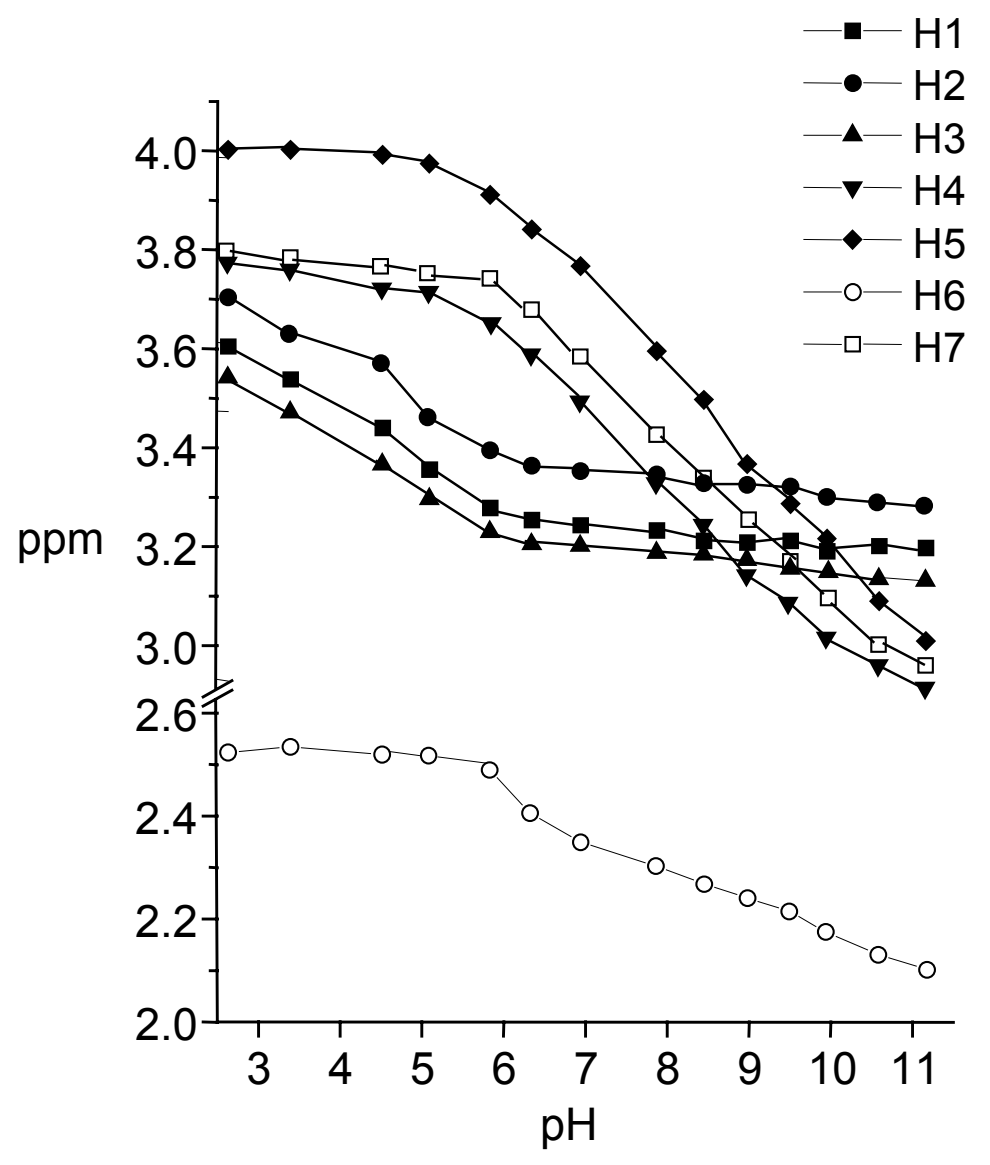

$\mathrm{a}$

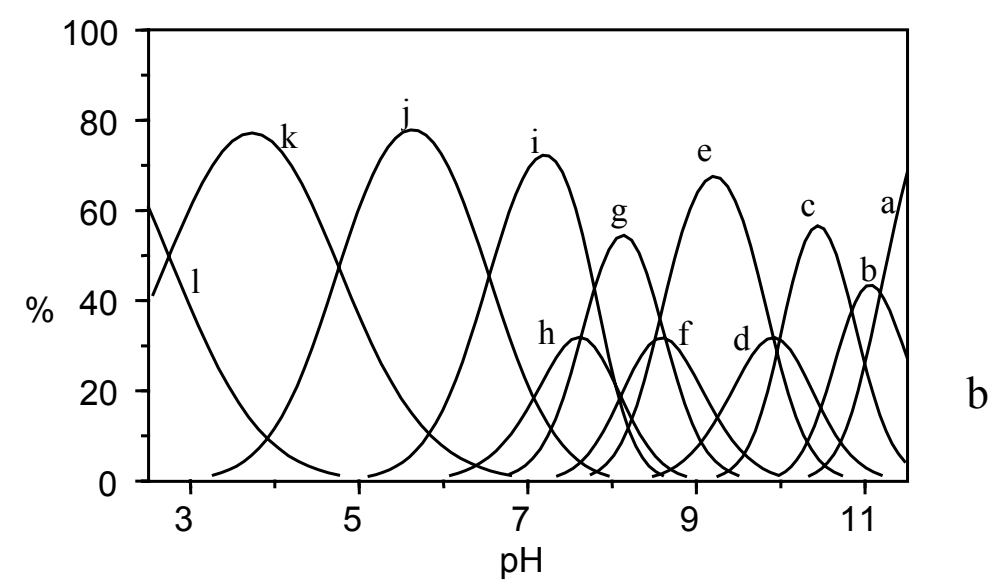

Figure S2. (a) $\mathrm{pH}$ dependence of the ${ }^{1} \mathrm{H}$ NMR signals of $\mathbf{L 2}$; (b) and distribution diagram of the $\mathbf{L} 2$ protonated species $\left(\mathrm{a}=\mathbf{L} \mathbf{2}, \mathrm{b}=\mathrm{HL2}^{+}, \mathrm{c}=\mathrm{H}_{2} \mathbf{L} \mathbf{2}^{2+}, \mathrm{d}=\mathrm{H}_{3} \mathbf{L} \mathbf{2}^{3+}, \mathrm{e}=\mathrm{H}_{4} \mathbf{L} \mathbf{2}^{4+}, \mathrm{f}=\mathrm{H}_{5} \mathbf{L} \mathbf{2} \mathrm{A}^{5+}\right.$, $\left.\mathrm{g}=\mathrm{H}_{6} \mathbf{L} 2^{6+}, \mathrm{h}=\mathrm{H}_{7} \mathbf{L} \mathbf{2}^{7+}, \mathrm{i}=\mathrm{H}_{8} \mathbf{L} \mathbf{2}^{8+}, \mathrm{j}=\mathrm{H}_{9} \mathbf{L} 2^{9+}, \mathrm{k}=\mathrm{H}_{10} \mathbf{L} 2^{10+}, 1=\mathrm{H}_{11} \mathbf{L} 2^{11+}\right)$. 

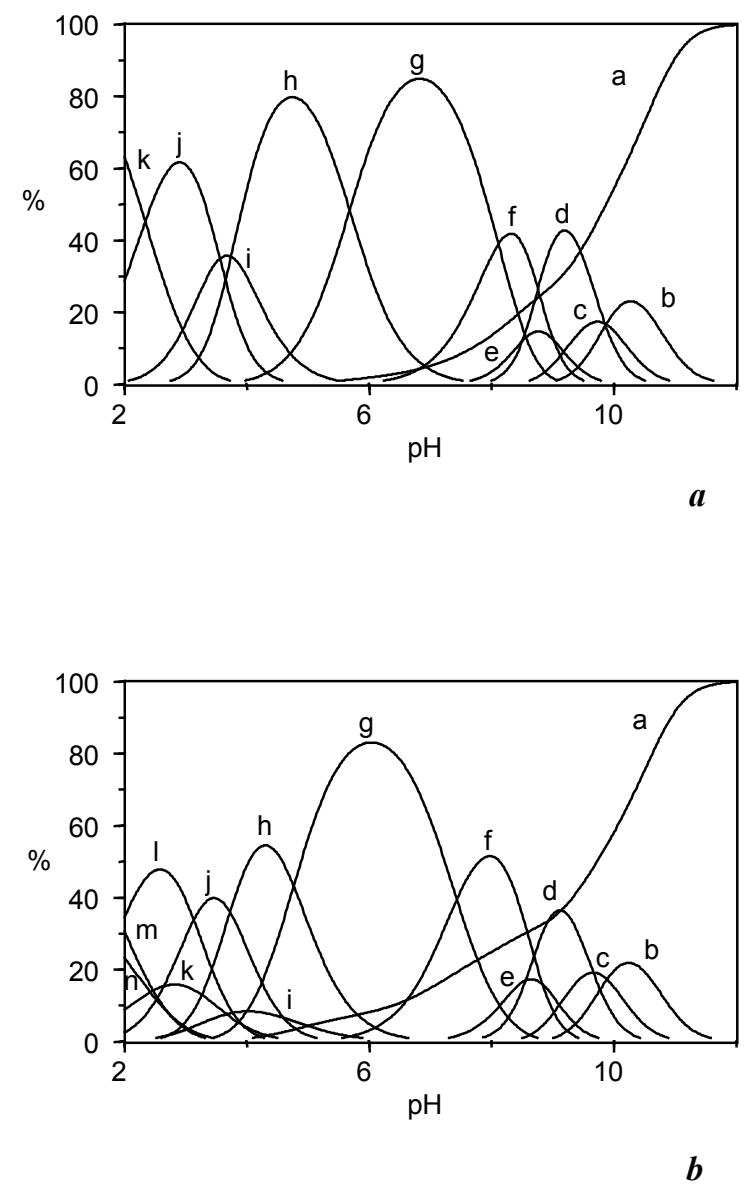

Figure S3. Distribution diagrams of the complexes of $\mathbf{L 1}$ with $(a)$ 1,2,3-BTC $\left(\mathrm{a}=\mathrm{A}^{3-}, \mathrm{b}=\right.$ $\mathrm{H}_{2} \mathbf{L 1 A}^{-}, \mathrm{c}=\mathrm{H}_{3} \mathbf{L} 1 \mathrm{~A}, \mathrm{~d}=\mathrm{H}_{4} \mathbf{L} 1 \mathrm{~A}^{+}, \mathrm{e}=\mathrm{H}_{5} \mathbf{L 1}^{2+}, \mathrm{f}=\mathrm{H}_{6} \mathbf{L 1}^{3+}, \mathrm{g}=\mathrm{H}_{7} \mathbf{L} \mathbf{1} \mathrm{A}^{4+}, \mathrm{h}=\mathrm{H}_{8} \mathbf{L} \mathbf{1} \mathrm{A}^{5+}, \mathrm{i}$ $\left.=\mathrm{H}_{9} \mathbf{L} \mathbf{1} \mathrm{A}^{6+}, \mathrm{j}=\mathrm{H}_{10} \mathbf{L} \mathbf{1} \mathrm{A}^{7+}, \mathrm{k}=\mathrm{H}_{11} \mathbf{L} \mathbf{1} \mathrm{A}^{8+}, \mathrm{A}=1,2,3-\mathrm{BTC}\right)$ and $(b)$ with 1,2,4-BTC $\left(\mathrm{a}=\mathrm{A}^{3-}\right.$, $\mathrm{b}=\mathrm{H}_{2} \mathbf{L} \mathbf{1} \mathrm{A}^{-}, \mathrm{c}=\mathrm{H}_{3} \mathbf{L} \mathbf{1} \mathrm{A}, \mathrm{d}=\mathrm{H}_{4} \mathbf{L} \mathbf{1} \mathrm{A}^{+}, \mathrm{e}=\mathrm{H}_{5} \mathbf{L 1}^{2+}, \mathrm{f}=\mathrm{H}_{6} \mathbf{L 1}^{3+}, \mathrm{g}=\mathrm{H}_{7} \mathbf{L} \mathbf{1} \mathrm{A}^{4+}, \mathrm{h}=$ $\mathrm{H}_{8} \mathbf{L 1}^{5+}, \mathrm{i}=\mathrm{HA}^{2-}, \mathrm{j}=\mathrm{H}_{9} \mathbf{L} \mathbf{1} \mathrm{A}^{6+}, \mathrm{k}=\mathrm{H}_{2} \mathrm{~A}^{-}, \mathrm{l}=\mathrm{H}_{10} \mathbf{L} \mathbf{1} \mathrm{A}^{7+}, \mathrm{m}=\mathrm{H}_{11} \mathbf{L 1}^{8+}, \mathrm{n}=\mathrm{H}_{\mathrm{a}} \mathrm{A}$ ) 

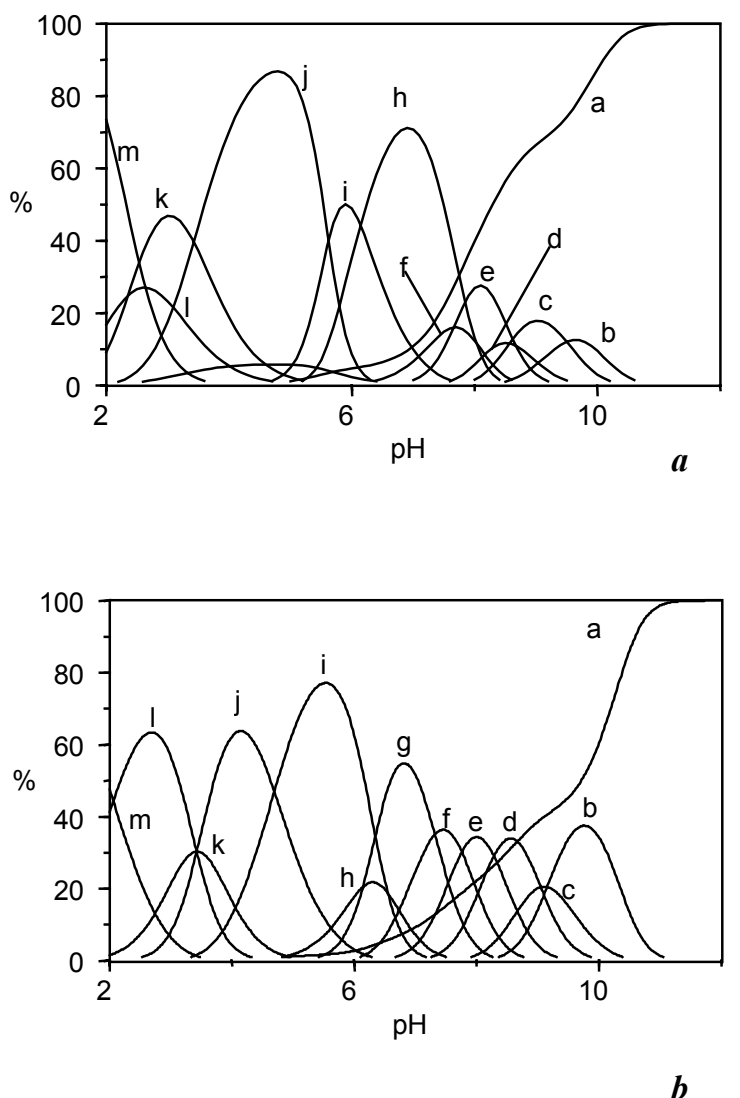

Figure S4. Distribution diagrams of the complexes $(a)$ of $1,2,3-\mathrm{BTC}$ with $\mathbf{L 2}\left(\mathrm{a}=\mathrm{A}^{3-}, \mathrm{b}=\right.$ $\mathrm{H}_{3} \mathbf{L} \mathbf{2} \mathrm{A}, \mathrm{c}=\mathrm{H}_{4} \mathbf{L} \mathbf{2} \mathrm{A}^{+}, \mathrm{d}=\mathrm{H}_{5} \mathbf{L} \mathbf{2} \mathrm{A}^{2+}, \mathrm{e}=\mathrm{H}_{6} \mathbf{L} \mathbf{2} \mathrm{A}^{3+}, \mathrm{f}=\mathrm{H}_{7} \mathbf{L} \mathbf{2} \mathrm{A}^{4+}, \mathrm{g}=\mathrm{H}_{8} \mathbf{L} \mathbf{2} \mathrm{A}^{5+}, \mathrm{h}=\mathrm{H}_{9} \mathbf{L} \mathbf{2} \mathrm{A}^{6+}$, $\left.\mathrm{i}=\mathrm{H}_{10} \mathbf{L} \mathbf{2} \mathrm{A}^{7+}, \mathrm{j}=\mathrm{H}_{11} \mathbf{L} \mathbf{2} \mathrm{A}^{8+}, \mathrm{k}=\mathrm{H}_{11} \mathbf{L} \mathbf{2} \mathrm{A}^{8+}, 1=\mathrm{H}_{2} \mathrm{~A}^{-}, \mathrm{m}=\mathrm{H}_{3} \mathrm{~A}, \mathrm{~A}=1,2,3-\mathrm{BTC}\right)$ and $(b)$ of 1,2,4-BTC with $\mathbf{L} 2\left(\mathrm{a}=\mathrm{A}^{3-}, \mathrm{b}=\mathrm{H}_{3} \mathbf{L} \mathbf{2} \mathrm{A}, \mathrm{c}=\mathrm{H}_{4} \mathbf{L} \mathbf{2} \mathrm{A}^{+}, \mathrm{d}=\mathrm{H}_{5} \mathbf{L} \mathbf{2} \mathrm{A}^{2+}, \mathrm{e}=\mathrm{H}_{6} \mathbf{L} \mathbf{2} \mathrm{A}^{3+}, \mathrm{f}=\right.$ $\mathrm{H}_{7} \mathbf{L} \mathbf{2} \mathrm{A}^{4+}, \mathrm{g}=\mathrm{H}_{8} \mathbf{L} \mathbf{2} \mathrm{A}^{5+}, \mathrm{h}=\mathrm{H}_{9} \mathbf{L} \mathbf{2} \mathrm{A}^{6+}, \mathrm{i}=\mathrm{H}_{10} \mathbf{L} \mathbf{2} \mathrm{A}^{7+}, \mathrm{j}=\mathrm{H}_{11} \mathbf{L} \mathbf{2} \mathrm{A}^{8+}, \mathrm{k}=\mathrm{H}_{12} \mathbf{L} \mathbf{2} \mathrm{A}^{9+}, 1=$ $\left.\mathrm{H}_{13} \mathbf{L} \mathbf{2} \mathrm{A}^{10+}, \mathrm{m}=\mathrm{H}_{14} \mathbf{L} \mathbf{2} \mathrm{A}^{11+}\right)$ 

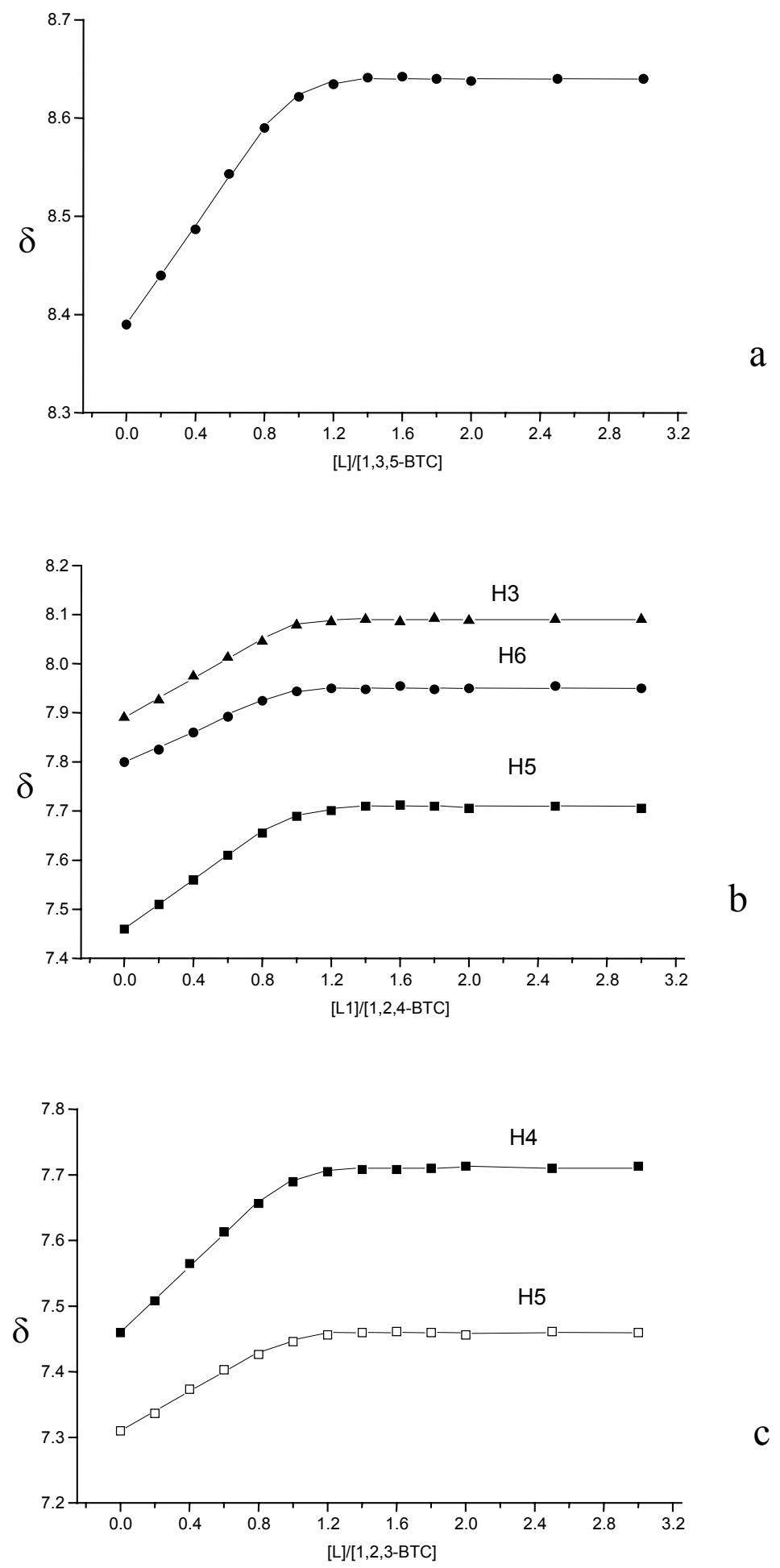

Figure S5. Plots of the chemical shifts $(\delta, \mathrm{ppm})$ of the ${ }^{1} \mathrm{H}$ NMR signals of $1,3,5$-BTC (a), 1,2,4BTC (b) and 1,2,3-BTC in the presence of increasing amount of $\mathbf{L 1}$ at $\mathrm{pH} 7$ and $298 \mathrm{~K}$ 

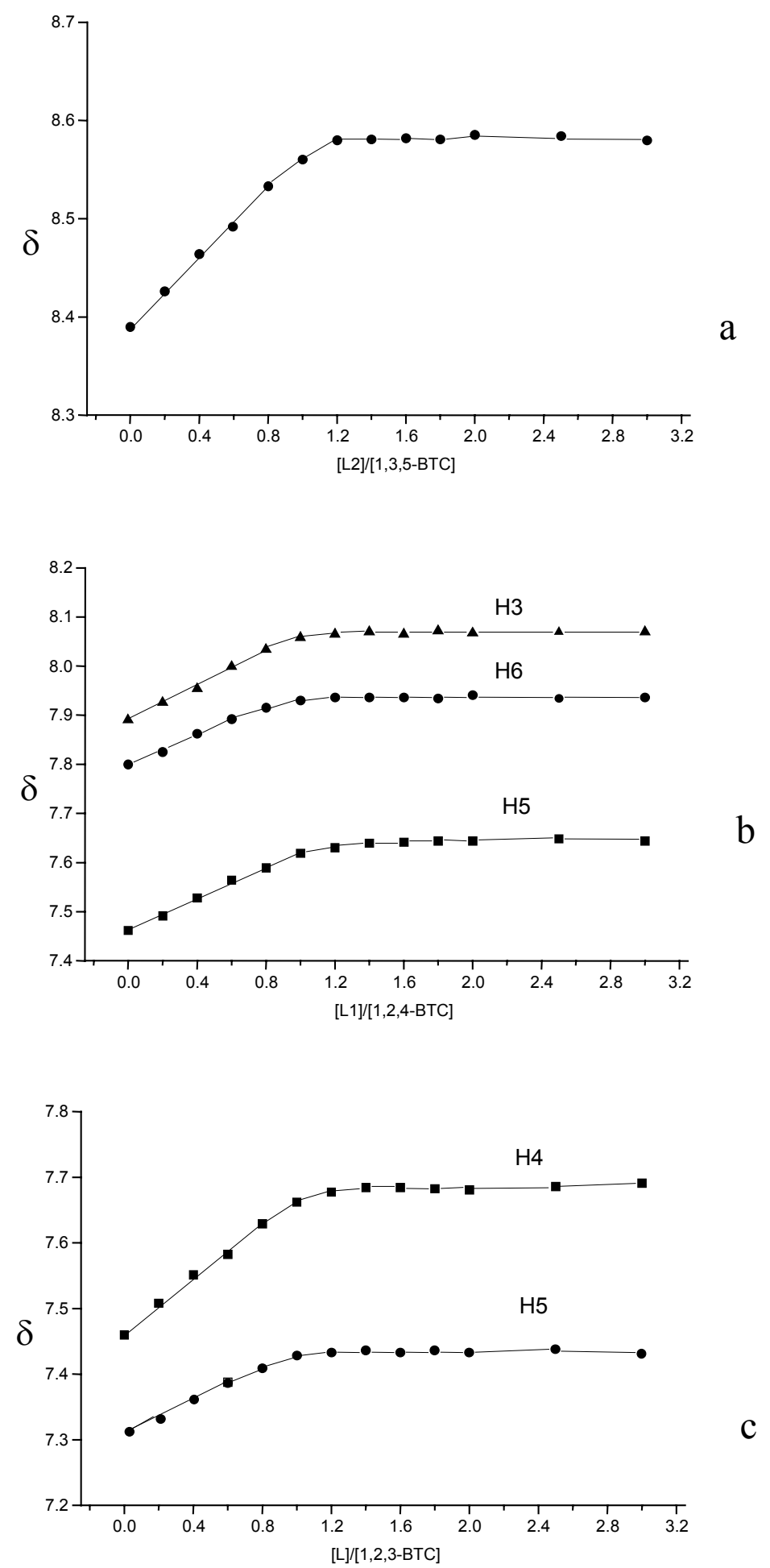

Figure S6. Plots of the chemical shifts $(\delta, \mathrm{ppm})$ of the ${ }^{1} \mathrm{H}$ NMR signals of 1,3,5-BTC (a), 1,2,4BTC (b) and 1,2,3-BTC in the presence of increasing amount of $\mathbf{L} 2$ at $\mathrm{pH} 7$ and $298 \mathrm{~K}$ 
Table S1. Energy content of the lowest energy conformers of the $\left[\mathrm{H}_{n} \mathbf{L}\right]^{\mathrm{n}+}$ free receptors, BTC ${ }^{3-}$ free anions and $\left[\mathrm{H}_{\mathrm{n}} \mathbf{L}(\mathrm{A})\right]^{(\mathrm{n}-3)+}$ adducts. For the $\left[\mathrm{H}_{7} \mathbf{L} \mathbf{1} \mathrm{A}\right]^{4+}$ adducts, the energy content of both conformers with endo and exo orientation of the bridgehead N1 nitrogen have been reported.

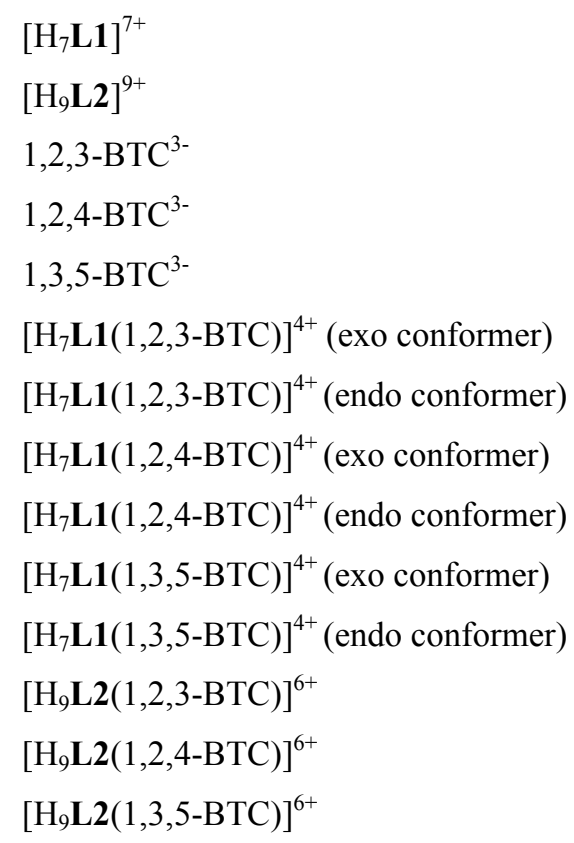

420.91 
Table S2. Cartesian coordinates, atomic charges and atom types for the lowest energy conformer of the $\left[\mathrm{H}_{7} \mathbf{L 1}\right]^{7+}$ protonated receptor

\begin{tabular}{|c|c|c|c|c|c|}
\hline atom & $\mathrm{x}$ & Y & $\mathrm{z}$ & $\begin{array}{l}\text { Atomic } \\
\text { charge }\end{array}$ & $\begin{array}{l}\text { Atom } \\
\text { type }\end{array}$ \\
\hline $\mathrm{C} 1$ & 0.1773408 & 1.441329 & 4.154705 & -0.05337095 & $\mathrm{CT} 2$ \\
\hline $\mathrm{C} 2$ & -0.1889397 & 0.1568853 & 4.959596 & -0.249845 & CT2 \\
\hline N3A & 0.9046513 & -0.3070956 & 5.890269 & 0.678133 & NH3 \\
\hline C4 & 0.727792 & 0.03629499 & 7.356344 & -0.2751794 & $\mathrm{CT} 2$ \\
\hline$\overline{C 5}$ & 0.8014829 & 1.543786 & 7.733909 & -0.06621885 & $\mathrm{CT} 2$ \\
\hline N4 & 2.026908 & 2.195185 & 7.202114 & -0.113308 & $\mathrm{~N}$ \\
\hline $\mathrm{C7}$ & 2.024781 & 3.673942 & 7.344787 & -0.05412912 & $\mathrm{CT} 2$ \\
\hline $\mathrm{C} 8$ & 3.002453 & 4.299779 & 6.32169 & -0.2520242 & $\mathrm{CT} 2$ \\
\hline N3B & 2.483692 & 4.2323 & 4.907328 & 0.6741848 & NH3 \\
\hline $\mathrm{C} 10$ & 3.043373 & 3.103298 & 4.083473 & -0.2612348 & $\mathrm{CT} 2$ \\
\hline C11 & 2.066981 & 2.645868 & 2.963902 & -0.06888437 & $\mathrm{CT} 2$ \\
\hline N2 & 1.386591 & 1.331739 & 3.256829 & -0.04276609 & $\mathrm{~N}$ \\
\hline C13 & 1.232494 & 0.436024 & 2.051468 & -0.03432655 & CT2 \\
\hline C14 & 0.2314186 & 0.9053928 & 0.9488997 & -0.22226 & $\mathrm{CT} 2$ \\
\hline N1 & 0.2098824 & -0.03174777 & -0.2552536 & 0.5842428 & NH3 \\
\hline C16 & -0.7343878 & -1.221563 & -0.08031616 & -0.2057118 & CT2 \\
\hline C17 & 0.05508255 & 0.7043745 & -1.596179 & -0.2181406 & $\mathrm{CT} 2$ \\
\hline C18 & -1.297041 & 1.449664 & -1.849324 & -0.03724098 & CT2 \\
\hline C19 & -0.2247899 & -2.51242 & -0.7970438 & -0.03298759 & CT2 \\
\hline N2' & -1.057591 & -3.757732 & -0.6171562 & -0.04908085 & $\mathrm{~N}$ \\
\hline $\mathrm{N} 2^{\prime \prime}$ & -1.293201 & 2.263792 & -3.118666 & -0.04778862 & $\mathrm{~N}$ \\
\hline $\mathrm{C} 22$ & -1.048064 & -4.317515 & 0.7816183 & -0.06407785 & $\mathrm{CT} 2$ \\
\hline C23 & -1.094813 & -5.867706 & 0.828162 & -0.2673254 & $\mathrm{CT} 2$ \\
\hline$N 3 A^{\prime}$ & 0.2376873 & -6.480926 & 0.5030625 & 0.6912127 & NH3 \\
\hline $\mathrm{C} 25$ & 0.1769402 & -7.944008 & 0.1651371 & -0.2613049 & $\mathrm{CT} 2$ \\
\hline $\mathrm{C} 26$ & 0.6048847 & -8.240793 & -1.293377 & -0.06949663 & $\mathrm{CT} 2$ \\
\hline $\mathrm{N} 4^{\prime}$ & -0.4168765 & -7.802895 & -2.285512 & -0.09881878 & $\mathrm{~N}$ \\
\hline $\mathrm{C} 28$ & -0.06730579 & -6.55115 & -3.012073 & -0.09696531 & CT2 \\
\hline C29 & -1.243356 & -6.074354 & -3.89626 & -0.243495 & $\mathrm{CT} 2$ \\
\hline $\mathrm{N}^{3} \mathrm{~B}^{\prime}$ & -2.396053 & -5.521857 & -3.09864 & 0.664958 & NH3 \\
\hline C31 & -2.340355 & -4.043865 & -2.813601 & -0.2328668 & $\mathrm{CT} 2$ \\
\hline C32 & -2.407993 & -3.741775 & -1.29196 & -0.07336903 & CT2 \\
\hline C33 & -1.488234 & 1.423131 & -4.358033 & -0.05921888 & $\mathrm{CT} 2$ \\
\hline C34 & -0.7834269 & 2.015081 & -5.608497 & -0.2673492 & CT2 \\
\hline N3A' ${ }^{\prime}$ & 0.6922892 & 1.72301 & -5.607455 & 0.6899981 & NH3 \\
\hline C36 & 1.572633 & 2.856582 & -6.057533 & -0.2595048 & CT2 \\
\hline C37 & 2.376297 & 3.486552 & -4.88905 & -0.07017279 & $\mathrm{CT} 2$ \\
\hline $\mathrm{N} 4{ }^{\prime \prime}$ & 1.534314 & 4.182483 & -3.874574 & -0.1058259 & $\mathrm{~N}$ \\
\hline C39 & 1.122851 & 5.561197 & -4.256658 & -0.09642553 & $\mathrm{CT} 2$ \\
\hline $\mathrm{C} 40$ & 0.2114893 & 6.182988 & -3.174434 & -0.243248 & $\mathrm{CT} 2$ \\
\hline N3B' & -1.220415 & 5.733965 & -3.295665 & 0.6658454 & NH3 \\
\hline $\mathrm{C} 42$ & -1.656994 & 4.667515 & -2.326203 & -0.2319756 & CT2 \\
\hline $\mathrm{C43}$ & -2.252724 & 3.42823 & -3.051454 & -0.0737834 & CT2 \\
\hline H4 4 & -2.432489 & -6.027488 & -2.19089 & 0.08821476 & $\mathrm{HC}$ \\
\hline H45 & 0.8656382 & -6.35772 & 1.322584 & 0.08309817 & $\mathrm{HC}$ \\
\hline H4 6 & 1.16389 & -0.4485232 & -0.2887933 & 0.0732643 & $\mathrm{HC}$ \\
\hline $\mathrm{H} 47$ & 1.811275 & 0.07031199 & 5.555628 & 0.06254947 & $\mathrm{HC}$ \\
\hline H48 & 2.714404 & 5.127786 & 4.431997 & 0.08461463 & $\mathrm{HC}$ \\
\hline H49 & -2.560168 & 3.74363 & -4.075827 & 0.06770742 & $\mathrm{HA}$ \\
\hline H5O & 0.8631276 & 0.9096334 & -6.23247 & 0.08495611 & $\mathrm{HC}$ \\
\hline
\end{tabular}




\begin{tabular}{|c|c|c|c|c|c|}
\hline H51 & 0.9698142 & 1.463989 & -4.639398 & 0.07205957 & $\mathrm{HC}$ \\
\hline H52 & -1.365828 & 5.373971 & -4.260202 & 0.08921224 & $\mathrm{HC}$ \\
\hline H53 & -0.9502757 & 3.109277 & -5.660439 & 0.1070455 & $\mathrm{HA}$ \\
\hline H5 4 & 0.333154 & 2.251535 & 4.901957 & 0.06976426 & $\mathrm{HA}$ \\
\hline H55 & -0.7098799 & 1.739815 & 3.553328 & 0.05261821 & $\mathrm{HA}$ \\
\hline H56 & -0.3892707 & -0.6804895 & 4.260666 & 0.1210305 & $\mathrm{HA}$ \\
\hline H5 7 & -1.111314 & 0.3369648 & 5.547795 & 0.1257147 & $\mathrm{HA}$ \\
\hline H58 & 0.9460959 & -1.344552 & 5.82409 & 0.09445989 & $\mathrm{HC}$ \\
\hline H59 & 1.527881 & -0.5087475 & 7.90034 & 0.08837879 & $\mathrm{HA}$ \\
\hline $\mathrm{H} 60$ & -0.2483951 & -0.3811803 & 7.678765 & 0.1550126 & $\mathrm{HA}$ \\
\hline H61 & 0.7882506 & 1.63365 & 8.84322 & 0.1403791 & $\mathrm{HA}$ \\
\hline $\mathrm{H} 62$ & -0.1085785 & 2.048099 & 7.337235 & 0.113203 & $\mathrm{HA}$ \\
\hline H63 & 2.019318 & 2.029679 & 6.195479 & 0.0474239 & $\mathrm{H}$ \\
\hline H64 & 2.343576 & 3.9434 & 8.375188 & 0.05636787 & $\mathrm{HA}$ \\
\hline H65 & 1.007303 & 4.096345 & 7.17526 & 0.1479983 & $\mathrm{HA}$ \\
\hline H66 & 4.001916 & 3.82315 & 6.374687 & 0.1605394 & $\mathrm{HA}$ \\
\hline H67 & 3.125826 & 5.378218 & 6.558362 & 0.1216154 & $\mathrm{HA}$ \\
\hline H68 & 1.449891 & 4.129817 & 4.943923 & 0.06923229 & $\mathrm{HC}$ \\
\hline H69 & 3.293134 & 2.252347 & 4.748592 & 0.1016911 & $\mathrm{HA}$ \\
\hline $\mathrm{H} 70$ & 3.98245 & 3.477453 & 3.623885 & 0.1031891 & $\mathrm{HA}$ \\
\hline H71 & 2.696154 & 2.550748 & 2.046847 & 0.08695167 & $\mathrm{HA}$ \\
\hline $\mathrm{H} 72$ & 1.310522 & 3.4312 & 2.742901 & 0.09355211 & $\mathrm{HA}$ \\
\hline H73 & 0.9218097 & -0.5756365 & 2.393674 & 0.05496246 & $\mathrm{HA}$ \\
\hline H74 & 2.231382 & 0.3075065 & 1.576356 & 0.06854713 & $\mathrm{HA}$ \\
\hline H75 & 0.5402429 & 1.913118 & 0.6045643 & 0.1275005 & $\mathrm{HA}$ \\
\hline H76 & -0.7963592 & 0.9748828 & 1.355509 & 0.1090513 & $\mathrm{HA}$ \\
\hline H77 & -1.735055 & -0.9355395 & -0.4530256 & 0.1002657 & $\mathrm{HA}$ \\
\hline H78 & -0.8198956 & -1.439235 & 1.003596 & 0.1175704 & $\mathrm{HA}$ \\
\hline H79 & 0.2170346 & -0.04972604 & -2.391508 & 0.1257495 & $\mathrm{HA}$ \\
\hline $\mathrm{H} 8 \mathrm{O}$ & 0.8894255 & 1.433053 & -1.654352 & 0.1108775 & $\mathrm{HA}$ \\
\hline H81 & -1.460176 & 2.114928 & -0.9705718 & 0.05720091 & $\mathrm{HA}$ \\
\hline $\mathrm{H} 82$ & -2.13524 & 0.7190357 & -1.865565 & 0.06904674 & $\mathrm{HA}$ \\
\hline H83 & 0.8079239 & -2.721207 & -0.4372487 & 0.07337475 & $\mathrm{HA}$ \\
\hline H84 & -0.1381731 & -2.294165 & -1.884258 & 0.05375206 & $\mathrm{HA}$ \\
\hline $\mathrm{H} 85$ & -1.91341 & -3.914042 & 1.354442 & 0.08115172 & $\mathrm{HA}$ \\
\hline H86 & -0.1290739 & -3.996243 & 1.325794 & 0.07776952 & $\mathrm{HA}$ \\
\hline H87 & -1.848648 & -6.252504 & 0.1114443 & 0.112 & $\mathrm{HA}$ \\
\hline H88 & -1.36848 & -6.198807 & 1.851985 & 0.09983581 & $\mathrm{HA}$ \\
\hline H89 & 0.6423677 & -5.974156 & -0.3095537 & 0.07068992 & $\mathrm{HC}$ \\
\hline $\mathrm{H} 90$ & -0.8393074 & -8.342923 & 0.3586505 & 0.1574611 & $\mathrm{HA}$ \\
\hline H91 & 0.88149 & -8.455799 & 0.8553181 & 0.1057162 & $\mathrm{HA}$ \\
\hline H92 & 0.7039831 & -9.345621 & -1.382981 & 0.03116882 & $\mathrm{HA}$ \\
\hline H93 & 1.609705 & -7.810457 & -1.505203 & 0.1096033 & $\mathrm{HA}$ \\
\hline H94 & -0.4725337 & -8.538024 & -2.994561 & 0.1399574 & $\mathrm{H}$ \\
\hline H95 & 0.8143197 & -6.740699 & -3.665008 & 0.1163316 & $\mathrm{HA}$ \\
\hline H96 & 0.2051506 & -5.745329 & -2.295237 & 0.1250473 & $\mathrm{HA}$ \\
\hline H97 & -1.64417 & -6.926205 & -4.485756 & 0.09912068 & $\mathrm{HA}$ \\
\hline H98 & -0.8992855 & -5.300463 & -4.612252 & 0.149464 & $\mathrm{HA}$ \\
\hline H99 & -3.272881 & -5.715874 & -3.62277 & 0.04560477 & $\mathrm{HC}$ \\
\hline $\mathrm{H} 100$ & -3.226568 & -3.595371 & -3.31093 & 0.1370711 & $\mathrm{HA}$ \\
\hline $\mathrm{H} 101$ & -1.430773 & -3.603831 & -3.266335 & 0.110602 & $\mathrm{HA}$ \\
\hline $\mathrm{H} 102$ & -2.899047 & -2.754019 & -1.145498 & 0.09903866 & $\mathrm{HA}$ \\
\hline $\mathrm{H} 103$ & -3.087001 & -4.493239 & -0.8261167 & 0.0666759 & $\mathrm{HA}$ \\
\hline $\mathrm{H} 104$ & -2.57392 & 1.30349 & -4.577138 & 0.09257048 & $\mathrm{HA}$ \\
\hline H105 & -1.085875 & 0.3941236 & -4.208867 & 0.07361388 & $\mathrm{HA}$ \\
\hline H106 & -1.20298 & 1.548918 & -6.5245 & 0.1066843 & $\mathrm{HA}$ \\
\hline H107 & 0.969086 & 3.621402 & -6.584067 & 0.1575159 & $\mathrm{HA}$ \\
\hline H108 & 2.289 & 2.425865 & -6.789493 & 0.1099333 & $\mathrm{HA}$ \\
\hline H109 & 3.138165 & 4.18394 & -5.303611 & 0.03645563 & $\mathrm{HA}$ \\
\hline H110 & 2.934545 & 2.673592 & -4.373264 & 0.1145592 & $\mathrm{HA}$ \\
\hline
\end{tabular}




\begin{tabular}{|l|l|l|l|l|l|}
\hline H111 & 2.112549 & 4.283579 & -3.037254 & 0.1369781 & H \\
\hline H112 & 0.5916907 & 5.551717 & -5.234775 & 0.1194178 & HA \\
\hline H113 & 2.028957 & 6.199381 & -4.365679 & 0.1210709 & HA \\
\hline H114 & 0.2017032 & 7.286145 & -3.310871 & 0.09276158 & HA \\
\hline H115 & 0.5855096 & 5.973487 & -2.15286 & 0.146783 & HA \\
\hline H116 & -1.830794 & 6.564218 & -3.156072 & 0.04785198 & HC \\
\hline H117 & -2.442482 & 5.125672 & -1.688438 & 0.1349651 & HA \\
\hline H118 & -0.808912 & 4.378638 & -1.675147 & 0.1149876 & HA \\
\hline H119 & -3.191228 & 3.125386 & -2.532908 & 0.08094525 & HA \\
\hline
\end{tabular}

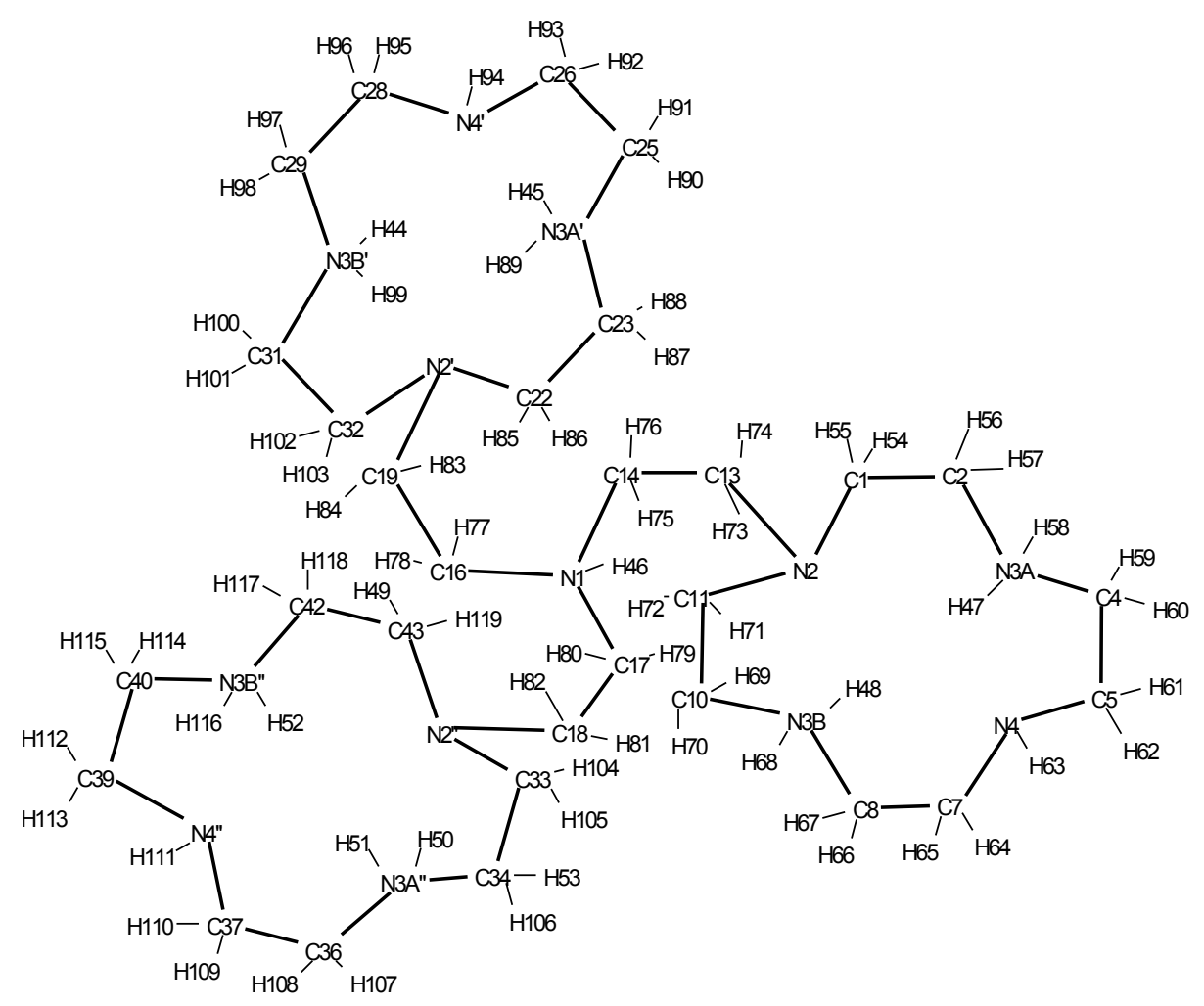

Drawing of the $\left[\mathrm{H}_{7} \mathbf{L} \mathbf{1}\right]^{7+}$ protonated receptor with atom labelling 
Table S3. Cartesian coordinates, atomic charges and atom types for the lowest energy conformer of

\section{the $\left[\mathrm{H}_{9} \mathbf{L 2}\right]^{9+}$ protonated receptor}

\begin{tabular}{|c|c|c|c|c|c|}
\hline atom & $\mathrm{x}$ & Y & $\mathrm{z}$ & charge & $\begin{array}{l}\text { Atom } \\
\text { type }\end{array}$ \\
\hline N1 & 0.03423944 & 0.5080559 & 0.1669819 & -0.02972317 & $\mathrm{~N}$ \\
\hline $\mathrm{C} 2$ & 0.05701784 & 0.9446017 & 1.610873 & -0.07327127 & CT2 \\
\hline C3 & 1.438378 & 0.6943829 & 2.290468 & -0.07826853 & $\mathrm{CT} 2$ \\
\hline $\mathrm{N} 2$ & 1.484059 & 1.176533 & 3.71903 & -0.0346899 & $\mathrm{~N}$ \\
\hline C5 & 1.705902 & 2.667662 & 3.821077 & -0.07325888 & CT2 \\
\hline C6 & 1.02319 & 3.311223 & 5.06045 & -0.2438774 & CT2 \\
\hline N3A & 1.967394 & 3.432549 & 6.222995 & 0.6754704 & NH3 \\
\hline $\mathrm{C} 8$ & 1.408369 & 2.973945 & 7.538743 & -0.2336526 & CT2 \\
\hline C9 & 2.536333 & 2.48784 & 8.474504 & -0.07792711 & CT2 \\
\hline C10 & 2.045142 & 1.753429 & 9.745188 & -0.2388687 & CT2 \\
\hline $\mathrm{N} 4$ & 3.052384 & 0.743728 & 10.23393 & 0.676497 & NH3 \\
\hline C12 & 2.675565 & -0.7035124 & 10.02339 & -0.2380052 & CT2 \\
\hline C13 & 3.125546 & -1.270351 & 8.65092 & -0.09078741 & CT2 \\
\hline C14 & 2.049266 & -1.229872 & 7.542591 & -0.2368579 & CT2 \\
\hline N3B & 2.636179 & -1.546319 & 6.194915 & 0.674334 & NH3 \\
\hline C16 & 1.864356 & -0.9724609 & 5.038607 & -0.24617 & CT2 \\
\hline C17 & 2.451638 & 0.38482 & 4.56288 & -0.0649147 & CT2 \\
\hline C18 & 0.648701 & 1.534695 & -0.7516692 & -0.08154106 & CT2 \\
\hline C19 & 1.299297 & 0.9001099 & -2.017537 & -0.06642246 & $\mathrm{CT} 2$ \\
\hline $\mathrm{N} 2^{\prime}$ & 2.098687 & 1.896554 & -2.812636 & -0.03678226 & $\mathrm{~N}$ \\
\hline $\mathrm{C} 21$ & 3.345495 & 1.333072 & -3.449537 & -0.06388426 & CT2 \\
\hline $\mathrm{C} 22$ & 3.142429 & 0.2803198 & -4.579501 & -0.2748022 & CT2 \\
\hline N3A' & 4.358038 & 0.1905527 & -5.456283 & 0.6857367 & NH3 \\
\hline C24 & 4.056851 & 0.09125008 & -6.926499 & -0.2373142 & $\mathrm{CT} 2$ \\
\hline $\mathrm{C} 25$ & 5.243942 & 0.5569184 & -7.807312 & -0.1048369 & $\mathrm{CT} 2$ \\
\hline $\mathrm{C} 26$ & 5.540663 & 2.078851 & -7.813906 & -0.2616625 & CT2 \\
\hline $\mathrm{N} 4^{\prime}$ & 4.49643 & 2.86929 & -8.562204 & 0.6882553 & NH3 \\
\hline $\mathrm{C} 28$ & 4.093473 & 4.16216 & -7.904512 & -0.2451124 & CT2 \\
\hline $\mathrm{C} 29$ & 2.879996 & 3.982607 & -6.962852 & -0.08162355 & CT2 \\
\hline C30 & 2.603453 & 5.192383 & -6.039796 & -0.23669 & $\mathrm{CT} 2$ \\
\hline N3B' & 1.486384 & 4.932851 & -5.061434 & 0.6768327 & NH3 \\
\hline C32 & 1.895748 & 4.246364 & -3.784857 & -0.252038 & CT2 \\
\hline C33 & 1.285307 & 2.823291 & -3.681303 & -0.05466318 & CT2 \\
\hline C34 & -1.338565 & 0.07828319 & -0.2857125 & -0.06994438 & CT2 \\
\hline C35 & -1.690577 & -1.375442 & 0.1559478 & -0.0857563 & $\mathrm{CT} 2$ \\
\hline $\mathrm{N} 2^{\prime \prime}$ & -3.094043 & -1.780556 & -0.2158702 & -0.03302765 & $\mathrm{~N}$ \\
\hline C37 & -4.136545 & -1.234154 & 0.7282675 & -0.07618761 & $\mathrm{CT} 2$ \\
\hline C38 & -5.480428 & -0.9287325 & 0.0109223 & -0.2380457 & $\mathrm{CT} 2$ \\
\hline $\mathrm{N}^{2} \mathrm{~A}^{\prime \prime}$ & -6.67485 & -1.309731 & 0.8457403 & 0.6795688 & NH3 \\
\hline C40 & -7.394827 & -2.554901 & 0.3962921 & -0.2530513 & $\mathrm{CT} 2$ \\
\hline C41 & -8.592756 & -2.240141 & -0.5322421 & -0.1104765 & CT2 \\
\hline C42 & -9.246887 & -3.478118 & -1.200456 & -0.2995214 & CT2 \\
\hline $\mathrm{N} 4^{\prime \prime}$ & -8.815643 & -3.690542 & -2.63083 & 0.7936053 & NH3 \\
\hline C44 & -7.592366 & -4.549821 & -2.817833 & -0.3162179 & CT2 \\
\hline C45 & -6.35581 & -3.713847 & -3.227633 & -0.1251726 & CT2 \\
\hline $\mathrm{C} 46$ & -5.001352 & -4.416891 & -2.981506 & -0.2534809 & CT2 \\
\hline $\mathrm{N} 3 \mathrm{~B}^{\prime \prime}$ & -3.880567 & -3.416846 & -2.873679 & 0.6655893 & NH3 \\
\hline $\mathrm{C} 48$ & -2.835272 & -3.744668 & -1.841313 & -0.2427883 & CT2 \\
\hline C49 & -3.225486 & -3.271484 & -0.4120548 & -0.0516839 & $\mathrm{CT} 2$ \\
\hline $\mathrm{H} 50$ & -0.2279048 & 2.016154 & 1.696258 & 0.04068887 & $\mathrm{HA}$ \\
\hline H51 & -0.6852241 & 0.3639292 & 2.206563 & 0.06131124 & $\mathrm{HA}$ \\
\hline H52 & 2.251271 & 1.167545 & 1.697016 & 0.05268669 & $\mathrm{HA}$ \\
\hline
\end{tabular}




\begin{tabular}{|c|c|c|c|c|c|}
\hline H53 & 1.602399 & -0.4076584 & 2.245592 & 0.07904267 & $\mathrm{HA}$ \\
\hline H54 & 1.255343 & 3.183481 & 2.940059 & 0.09548759 & $\mathrm{HA}$ \\
\hline H55 & 2.792643 & 2.906554 & 3.797093 & 0.05798876 & $\mathrm{HA}$ \\
\hline H5 6 & 0.1269333 & 2.727493 & 5.350534 & 0.09112895 & $\mathrm{HA}$ \\
\hline H57 & 0.6995614 & 4.342579 & 4.804976 & 0.1328356 & $\mathrm{HA}$ \\
\hline H58 & 2.251446 & 4.428737 & 6.314122 & 0.0928117 & $\mathrm{HC}$ \\
\hline H59 & 2.81171 & 2.86263 & 6.013297 & 0.05848199 & $\mathrm{HC}$ \\
\hline $\mathrm{H} 60$ & 0.6928684 & 2.144819 & 7.354616 & 0.128697 & $\mathrm{HA}$ \\
\hline H61 & 0.8681023 & 3.833101 & 7.988357 & 0.1596975 & $\mathrm{HA}$ \\
\hline H62 & 3.147581 & 3.364466 & 8.788117 & 0.1165229 & $\mathrm{HA}$ \\
\hline H63 & 3.200695 & 1.808031 & 7.894312 & 0.02329117 & $\mathrm{HA}$ \\
\hline H64 & 1.07292 & 1.24805 & 9.576158 & 0.125408 & $\mathrm{HA}$ \\
\hline H65 & 1.910695 & 2.489389 & 10.56658 & 0.1594397 & $\mathrm{HA}$ \\
\hline H66 & 3.951993 & 0.9186292 & 9.743149 & 0.06775004 & $\mathrm{HC}$ \\
\hline H67 & 1.585424 & -0.8188491 & 10.18614 & 0.129018 & $\mathrm{HA}$ \\
\hline H68 & 3.19411 & -1.273122 & 10.82526 & 0.1649846 & $\mathrm{HA}$ \\
\hline H69 & 3.426942 & -2.332242 & 8.804955 & 0.1348016 & $\mathrm{HA}$ \\
\hline $\mathrm{H} 70$ & 4.030205 & -0.7210897 & 8.307222 & 0.05696726 & $\mathrm{HA}$ \\
\hline H71 & 1.585802 & -0.2228728 & 7.500007 & 0.08791327 & $\mathrm{HA}$ \\
\hline H72 & 1.260395 & -1.985008 & 7.743761 & 0.150506 & $\mathrm{HA}$ \\
\hline H73 & 2.67439 & -2.579775 & 6.08681 & 0.09618306 & $\mathrm{HC}$ \\
\hline H7 4 & 3.604484 & -1.169241 & 6.161294 & 0.06245226 & $\mathrm{HC}$ \\
\hline H75 & 0.7979153 & -0.8683056 & 5.323585 & 0.1223497 & $\mathrm{HA}$ \\
\hline H76 & 1.932997 & -1.712281 & 4.21344 & 0.1268889 & $\mathrm{HA}$ \\
\hline H77 & 3.400631 & 0.2090091 & 4.007344 & 0.09039891 & $\mathrm{HA}$ \\
\hline H78 & 2.718068 & 0.9573936 & 5.480231 & 0.03220356 & $\mathrm{HA}$ \\
\hline H79 & -0.1090544 & 2.291306 & -1.053254 & 0.04061413 & $\mathrm{HA}$ \\
\hline $\mathrm{H} 80$ & 1.465013 & 2.084657 & -0.2274822 & 0.0730713 & $\mathrm{HA}$ \\
\hline H81 & 1.960301 & 0.07817093 & -1.655931 & 0.07961541 & $\mathrm{HA}$ \\
\hline H82 & 0.516009 & 0.4387291 & -2.657561 & 0.04303908 & $\mathrm{HA}$ \\
\hline H83 & 3.96718 & 0.8632567 & -2.653515 & 0.1106229 & $\mathrm{HA}$ \\
\hline H84 & 3.948426 & 2.177861 & -3.851405 & 0.0612464 & $\mathrm{HA}$ \\
\hline H85 & 2.256092 & 0.5417762 & -5.190258 & 0.1047436 & $\mathrm{HA}$ \\
\hline H8 6 & 2.985138 & -0.72378 & -4.13401 & 0.1323479 & $\mathrm{HA}$ \\
\hline H87 & 4.907718 & -0.646189 & -5.175364 & 0.0918588 & $\mathrm{HC}$ \\
\hline $\mathrm{H} 88$ & 4.937296 & 1.039638 & -5.299332 & 0.077429 & $\mathrm{HC}$ \\
\hline H89 & 3.158379 & 0.70004 & -7.156307 & 0.08395875 & $\mathrm{HA}$ \\
\hline H9O & 3.831381 & -0.9746622 & -7.141359 & 0.09788138 & $\mathrm{HA}$ \\
\hline H91 & 5.04716 & 0.2264853 & -8.852612 & 0.122016 & $\mathrm{HA}$ \\
\hline H92 & 6.165313 & 0.0251991 & -7.477179 & 0.1351894 & $\mathrm{HA}$ \\
\hline H93 & 6.507775 & 2.262519 & -8.328813 & 0.1663375 & $\mathrm{HA}$ \\
\hline H94 & 5.622276 & 2.455136 & -6.774234 & 0.1264263 & $\mathrm{HA}$ \\
\hline H95 & 4.866267 & 3.083536 & -9.510254 & 0.1173348 & $\mathrm{HC}$ \\
\hline H96 & 3.82666 & 4.863821 & -8.723899 & 0.1650115 & $\mathrm{HA}$ \\
\hline H97 & 4.969246 & 4.578417 & -7.365576 & 0.123183 & $\mathrm{HA}$ \\
\hline H98 & 3.053607 & 3.087741 & -6.324548 & 0.02660477 & $\mathrm{HA}$ \\
\hline H99 & 1.974195 & 3.786879 & -7.580486 & 0.1301081 & $\mathrm{HA}$ \\
\hline $\mathrm{H} 100$ & 2.2868 & 6.063614 & -6.652553 & 0.1617545 & $\mathrm{HA}$ \\
\hline $\mathrm{H} 101$ & 3.510865 & 5.481445 & -5.471077 & 0.130734 & $\mathrm{HA}$ \\
\hline $\mathrm{H} 102$ & 0.7720159 & 4.34287 & -5.533041 & 0.05374652 & $\mathrm{HC}$ \\
\hline H103 & 1.054027 & 5.845621 & -4.813618 & 0.09268856 & $\mathrm{HC}$ \\
\hline H1O4 & 1.512729 & 4.870235 & -2.949879 & 0.1401723 & $\mathrm{HA}$ \\
\hline H105 & 3.000631 & 4.218684 & -3.71453 & 0.09451795 & $\mathrm{HA}$ \\
\hline H106 & 1.155887 & 2.38082 & -4.693013 & 0.0319823 & $\mathrm{HA}$ \\
\hline H107 & 0.2624147 & 2.95103 & -3.256023 & 0.08952004 & $\mathrm{HA}$ \\
\hline $\mathrm{H} 108$ & -2.107583 & 0.7919412 & 0.0829109 & 0.03746855 & $\mathrm{HA}$ \\
\hline H109 & -1.399179 & 0.08106247 & -1.399014 & 0.06484807 & $\mathrm{HA}$ \\
\hline H110 & -0.9494597 & -2.035079 & -0.3534056 & 0.07876152 & $\mathrm{HA}$ \\
\hline H111 & -1.533711 & -1.495054 & 1.250347 & 0.05029011 & $\mathrm{HA}$ \\
\hline H112 & -3.797162 & -0.2665466 & 1.167566 & 0.1016854 & $\mathrm{HA}$ \\
\hline
\end{tabular}




\begin{tabular}{|l|l|l|l|l|l|}
\hline H113 & -4.286498 & -1.931459 & 1.582737 & 0.05371946 & HA \\
\hline H114 & -5.542956 & -1.432109 & -0.9734938 & 0.07510346 & HA \\
\hline H115 & -5.554663 & 0.1659336 & -0.1632066 & 0.1368921 & HA \\
\hline H116 & -7.350296 & -0.5194202 & 0.8303927 & 0.09633541 & HC \\
\hline H117 & -6.35284 & -1.454401 & 1.823788 & 0.08320987 & HC \\
\hline H118 & -7.763212 & -3.054095 & 1.318373 & 0.1657704 & HA \\
\hline H119 & -6.668734 & -3.23317 & -0.09516069 & 0.08530283 & HA \\
\hline H120 & -8.269608 & -1.522472 & -1.318705 & 0.07467294 & HA \\
\hline H121 & -9.376591 & -1.727441 & 0.07264155 & 0.1704077 & HA \\
\hline H122 & -10.34673 & -3.318386 & -1.237803 & 0.1883122 & HA \\
\hline H123 & -9.067804 & -4.410241 & -0.6286147 & 0.1602553 & HA \\
\hline H124 & -9.604017 & -4.133833 & -3.143505 & 0.0317179 & HC \\
\hline H125 & -7.836798 & -5.273199 & -3.62501 & 0.1925005 & HA \\
\hline H126 & -7.402907 & -5.123641 & -1.888225 & 0.1518524 & HA \\
\hline H127 & -6.371655 & -2.752686 & -2.665995 & 0.109655 & HA \\
\hline H128 & -6.436934 & -3.464725 & -4.310886 & 0.1852552 & HA \\
\hline H129 & -4.757215 & -5.091857 & -3.828415 & 0.1717122 & HA \\
\hline H130 & -5.043103 & -5.01609 & -2.047906 & 0.1176016 & HA \\
\hline H131 & -4.292378 & -2.492904 & -2.633404 & 0.009807467 & HC \\
\hline H132 & -3.415315 & -3.34527 & -3.800841 & 0.1009631 & HC \\
\hline H133 & -1.897401 & -3.248877 & -2.166288 & 0.1284122 & HA \\
\hline H134 & -2.663482 & -4.840447 & -1.846427 & 0.1417626 & HA \\
\hline H135 & -2.60529 & -3.819888 & 0.3331236 & 0.08303726 & HA \\
\hline H136 & -4.277299 & -3.600761 & -0.2426222 & 0.00459516 & HA \\
\hline H137 & 3.194437 & 0.895493 & 11.25297 & 0.1185169 & HC \\
\hline H138 & 3.648443 & 2.275935 & -8.660635 & 0.06171131 & HC \\
\hline H139 & -8.623915 & -2.759569 & -3.051398 & -0.03671861 & HC \\
\hline
\end{tabular}

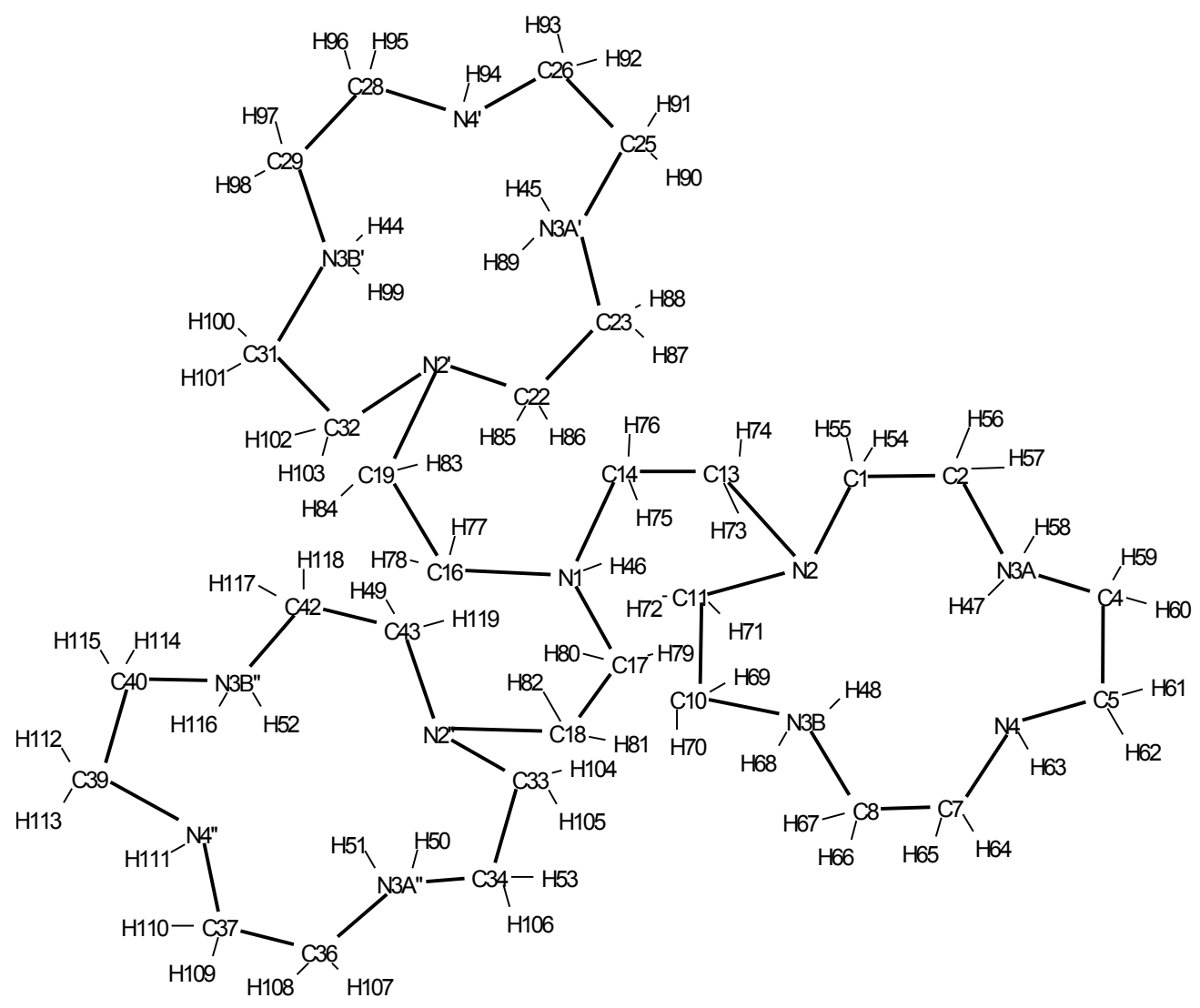

Drawing of the $\left[\mathrm{H}_{9} \mathbf{L} 2\right]^{9+}$ protonated receptor with atom labelling 
Table S4. Cartesian coordinates, atomic charges and atom types for the lowest energy conformer of the 1,3,5-BTC trianion

\begin{tabular}{|l|l|l|l|l|l|}
\hline & $x$ & $y$ & $z$ & $\begin{array}{l}\text { Atomic } \\
\text { charge }\end{array}$ & Atom type \\
\hline C1 & -1.235 & -0.728 & 2.462 & -0.16 & CA \\
\hline C2 & 1.201 & 0.684 & 2.462 & -0.043 & CA \\
\hline C3 & -0.003 & -1.41 & 2.462 & -0.043 & CA \\
\hline C4 & -1.215 & 0.68 & 2.462 & -0.043 & CA \\
\hline C5 & -0.008 & 1.406 & 2.462 & -0.16 & CA \\
\hline C6 & 1.226 & -0.724 & 2.462 & -0.16 & CA \\
\hline C7 & -2.45 & -1.433 & 2.462 & 0.439 & CA \\
\hline C8 & 2.444 & -1.424 & 2.462 & 0.439 & CA \\
\hline C9 & -0.011 & 2.811 & 2.462 & 0.439 & CA \\
\hline 01 & 2.498 & -2.687 & 2.462 & -0.677 & OC \\
\hline O2 & 3.563 & -0.834 & 2.462 & -0.677 & OC \\
\hline 03 & -1.081 & 3.485 & 2.462 & -0.677 & OC \\
\hline 04 & 1.056 & 3.489 & 2.462 & -0.677 & OC \\
\hline 05 & -2.499 & -2.696 & 2.462 & -0.677 & OC \\
\hline O6 & -3.571 & -0.848 & 2.462 & -0.677 & OC \\
\hline H16 & 2.139 & 1.228 & 2.462 & 0.119 & HA \\
\hline H17 & -0.001 & -2.494 & 2.462 & 0.119 & HA \\
\hline H18 & -2.155 & 1.22 & 2.462 & 0.119 & HA \\
\hline
\end{tabular}

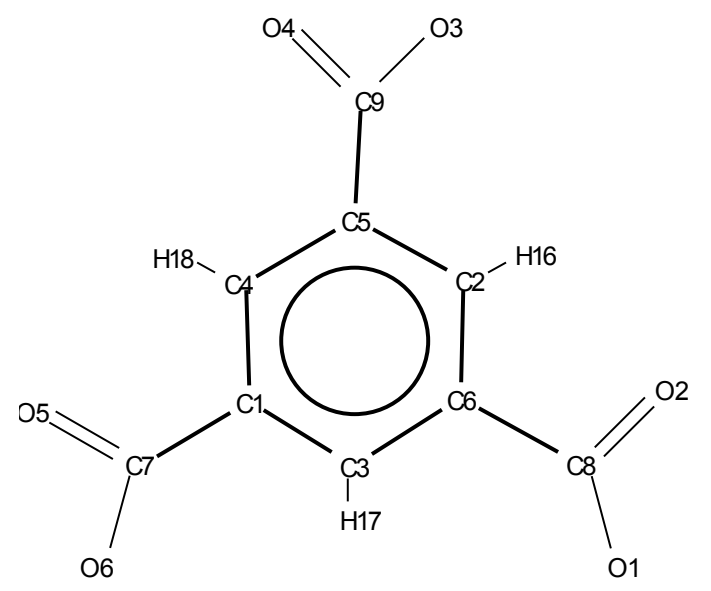

Drawing of the 1,3,5-BTC trianion with atom labelling 
Table S5. Cartesian coordinates, atomic charges and atom types for the lowest energy conformer of the $1,2,4-\mathrm{BTC}$ trianion

\begin{tabular}{|l|l|l|l|l|l|}
\hline & $\mathrm{X}$ & $\mathrm{Y}$ & $\mathrm{z}$ & $\begin{array}{l}\text { atomic } \\
\text { charge }\end{array}$ & Atom type \\
\hline C1 & -0.255 & -1.031 & -0.771 & -0.028 & CA \\
\hline C2 & -0.276 & 1.514 & 0.560 & -0.137 & CA \\
\hline C3 & 0.726 & -0.744 & 0.234 & -0.057 & CA \\
\hline C4 & -1.234 & -0.034 & -1.036 & -0.168 & CA \\
\hline C5 & -1.245 & 1.198 & -0.395 & -0.133 & CA \\
\hline C6 & 0.689 & 0.539 & 0.851 & -0.078 & CA \\
\hline H7 & -2.006 & -0.207 & -1.777 & 0.099 & HA \\
\hline H8 & -2.016 & 1.916 & -0.654 & 0.085 & HA \\
\hline H9 & 1.430 & 0.785 & 1.604 & 0.133 & HA \\
\hline C10 & -0.385 & -2.213 & -1.551 & 0.415 & CA \\
\hline C11 & 1.764 & -1.598 & 0.698 & 0.414 & CA \\
\hline C12 & -0.274 & 2.762 & 1.207 & 0.440 & CA \\
\hline O1 & -1.361 & -2.412 & -2.335 & -0.727 & OC \\
\hline O2 & 0.427 & -3.171 & -1.610 & -0.589 & OC \\
\hline O3 & 2.658 & -1.210 & 1.509 & -0.707 & OC \\
\hline O4 & 1.920 & -2.815 & 0.422 & -0.603 & OC \\
\hline O5 & 0.590 & 3.072 & 2.077 & -0.663 & OC \\
\hline O6 & -1.137 & 3.654 & 0.960 & -0.698 & OC \\
\hline
\end{tabular}

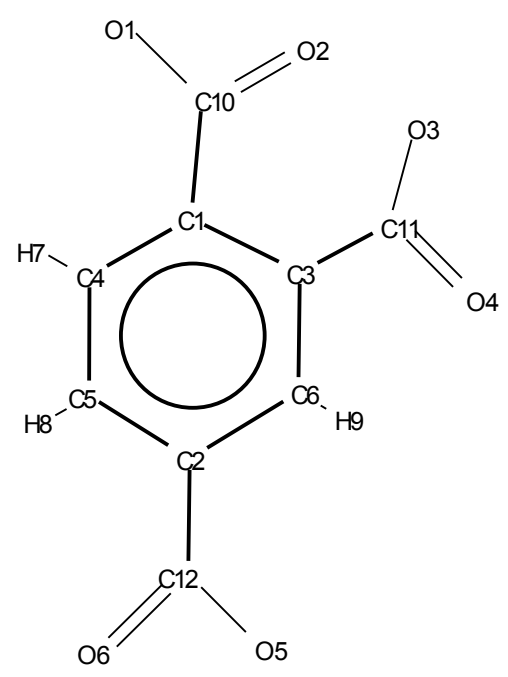

Drawing of the 1,2,4-BTC trianion with atom labelling 
Table S6. Cartesian coordinates, atomic charges and atom types for the lowest energy conformer of the 1,2,3-BTC trianion

\begin{tabular}{|l|l|l|l|l|l|}
\hline & $x$ & $y$ & $z$ & $\begin{array}{l}\text { atomic } \\
\text { charge }\end{array}$ & Atom type \\
\hline C1 & 0.009 & 0.089 & 2.063 & -0.246 & CA \\
\hline C2 & 1.194 & -0.071 & 1.368 & -0.137 & CA \\
\hline C3 & -1.181 & 0.2085374 & 1.368127 & -0.135 & CA \\
\hline C4 & -1.238 & 0.116 & -0.041 & -0.065 & CA \\
\hline C5 & 1.240 & -0.061 & -0.045 & -0.067 & CA \\
\hline C6 & 2.548 & 0.011 & -0.603 & 0.317 & CA \\
\hline C7 & -2.549 & 0.011 & -0.585 & 0.312 & CA \\
\hline O1 & 3.598 & -0.171 & 0.083 & -0.688 & OC \\
\hline O2 & 2.815 & 0.316 & -1.797 & -0.530 & OC \\
\hline O5 & -3.594 & 0.235 & 0.096 & -0.690 & OC \\
\hline O6 & -2.824 & -0.361 & -1.758 & -0.531 & OC \\
\hline C12 & -0.001 & 0.006 & -0.772 & 0.027 & CA \\
\hline C13 & -0.006 & -0.036 & -2.183 & 0.367 & CA \\
\hline O3 & -0.692 & 0.762 & -2.883 & -0.601 & OC \\
\hline 04 & 0.676 & -0.873 & -2.839 & -0.605 & OC \\
\hline H16 & -2.092 & 0.292 & 1.952 & 0.107 & HA \\
\hline H17 & 0.012 & 0.121 & 3.147 & 0.059 & HA \\
\hline H18 & 2.108 & -0.121 & 1.949 & 0.106 & HA \\
\hline
\end{tabular}

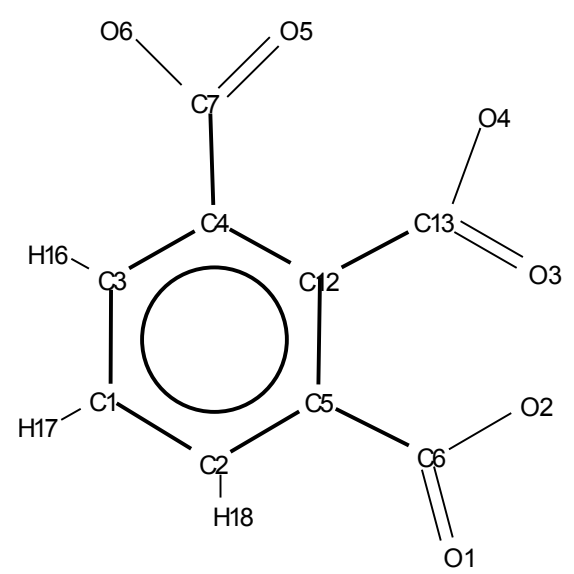

Drawing of the 1,2,3-BTC trianion with atom labelling 


\section{Description of the lowest energy conformer for the family with endo conformation of the N1 bridgehead nitrogen for the $\left[\mathrm{H}_{7} \mathrm{L1A}\right]^{4+} \operatorname{adduct}(\mathrm{A}=1,3,5-\mathrm{BTC})($ Figure S7 b)}

A full decription of the lowest energy conformer with exo conformation of the N1 bridgehead nitrogen for the $\left[\mathrm{H}_{7} \mathbf{L 1 A}\right]^{4+}$ adduct $(\mathrm{A}=1,3,5-\mathrm{BTC})$ (Figure $\left.\mathrm{S} 7 \mathrm{a}\right)$ is reported within the manuscript text. The lowest energy complex belonging to the family with endo conformation of the N1 bridgehead nitrogen (Figure S7 b) shows a bowl-shaped conformation of the receptor, similar to the conformer with exo conformation of the bridgehead nitrogen $\mathrm{N} 1$, the most significant difference being actually the endo orientation of the N1 capped nitrogen. The averaged distance between the cyclic $\mathrm{N}_{4}$ units, evaluated by measuring the distances between couple of symmetrically disposed $\mathrm{N} 3$ nitrogens belonging to different $\mathrm{N}_{4}$ moieties, is slightly larger (12 vs $11 \AA$ ) than in the former complex. Although the 1,3,5-BTC trianion is still encapsulated inside the cavity in a fashion similar to that observed in the former complex $(\mathrm{RMSD}=0.7)$, a different hydrogen bonding network is found; a single oxygen of each carboxylate function, in fact, gives rise to two hydrogen bonding contacts with two protonated $\mathrm{N} 3$ nitrogens of the same cyclic $\mathrm{N}_{4}$ unit, while the second oxygen does not give any hydrogen bonding (Figure S7 and Table S7). Most likely, such a different array of hydrogen bonds leads to the observed lower stability of this conformer $\left(22.8 \mathrm{~kJ} m o l^{-1}\right)$. 
Table S7. Hydrogen bond interactions $(\AA$, NH...O distance $d<2.4 \AA$ ) for the lowest energy conformer of the family with exo conformation of the N1 bridgehead nitrogen (a) and for lowest energy conformer of the family with endo conformation of the N1 bridgehead nitrogen (b) for the $\left[\mathrm{H}_{7} \mathbf{L} \mathbf{1 A}\right]^{4+}$ adduct $(\mathrm{A}=1,3,5-\mathrm{BTC})$.

(a)

$\begin{array}{ll}\text { N3AH....O6 } & 2.11 \\ \text { N3BH....O3 } & 1.95 \\ \text { N3A'H...O4 } & 2.18 \\ \text { N3B'H...O2 } & 2.16 \\ \text { N3A',H...O5 } & 2.07 \\ \text { N3B',H...O1 } & 2.16\end{array}$

(b)

$\begin{array}{ll}\text { N3AH....O1 } & 2.03 \\ \text { N3BH....O1 } & 1.98 \\ \text { N3A'H...O6 } & 2.04 \\ \text { N3B'H...O6 } & 1.99 \\ \text { N3A',H...O4 } & 2.02 \\ \text { N3B',H...O4 } & 1.99\end{array}$

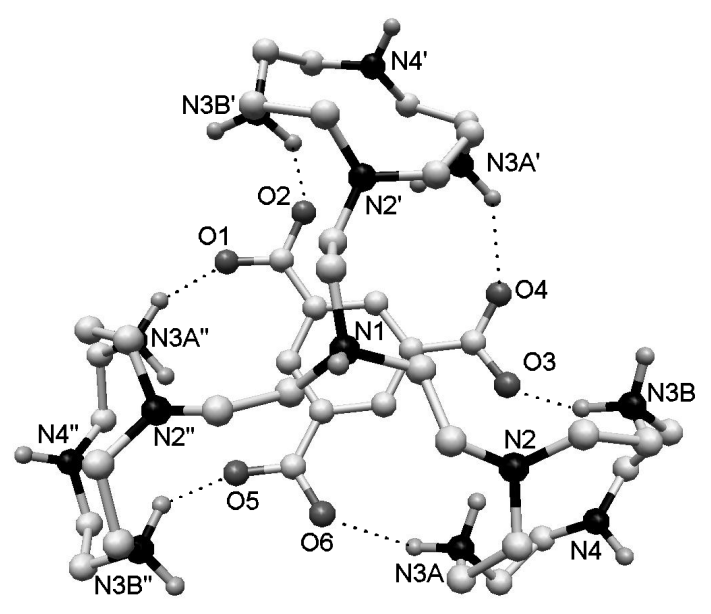

(a)

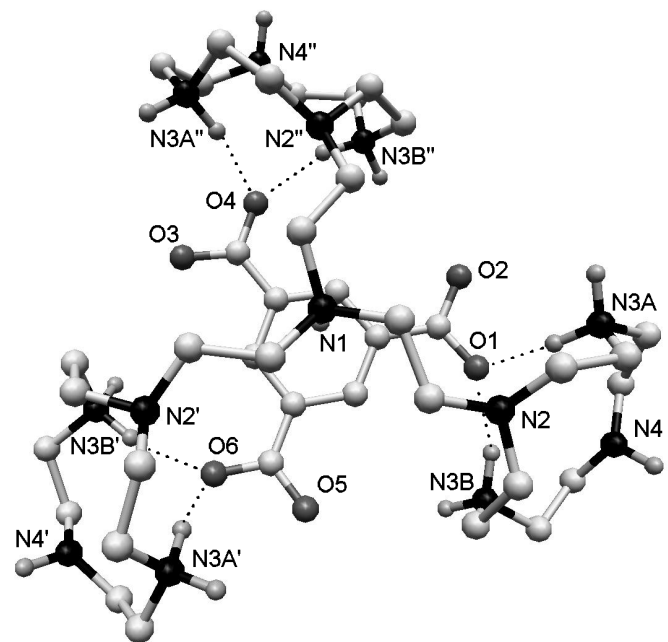

(b)

Figure S7. (a) lowest energy conformer of the family with exo conformation of the N1 bridgehead nitrogen for the $\left[\mathrm{H}_{7} \mathbf{L} \mathbf{1 A}\right]^{4+}$ adduct $(\mathrm{A}=1,3,5-\mathrm{BTC})$. (b) lowest energy conformer of the family with endo conformation of the $\mathrm{N} 1$ bridgehead nitrogen for the $\left[\mathrm{H}_{7} \mathbf{L} \mathbf{1} A\right]^{4+}$ adduct $(\mathrm{A}=1,3,5-\mathrm{BTC})$. 
Table S8. Cartesian coordinates for the lowest energy conformer of the complex $\left[\mathrm{H}_{7} \mathbf{L} \mathbf{1 A}\right]^{4+}(\mathrm{A}=$ 1,3,5-BTC) with exo conformation of the bridgehead N1 nitrogen (a)

\begin{tabular}{|c|c|c|c|}
\hline Atom & $\mathrm{x}$ & $\mathrm{y}$ & $\mathrm{z}$ \\
\hline $\mathrm{C} 1$ & 2.382 & -2.505 & 3.207 \\
\hline $\mathrm{C} 2$ & 3.583 & -1.542 & 2.936 \\
\hline N3A & 3.582 & -0.312 & 3.803 \\
\hline C4 & 4.37 & -0.412 & 5.083 \\
\hline C5 & 3.527 & 0.022 & 6.303 \\
\hline N4 & 2.521 & -1.025 & 6.607 \\
\hline C7 & 1.426 & -0.594 & 7.514 \\
\hline $\mathrm{C} 8$ & 0.208 & -1.542 & 7.393 \\
\hline N3B & -0.354 & -1.599 & 5.991 \\
\hline $\mathrm{C} 10$ & -0.047 & -2.879 & 5.264 \\
\hline C11 & -0.083 & -2.733 & 3.712 \\
\hline N2 & 1.002 & -1.904 & 3.057 \\
\hline C13 & 0.657 & -1.633 & 1.61 \\
\hline C14 & -0.265 & -0.393 & 1.438 \\
\hline N1 & -0.36 & 0.096 & -0.007 \\
\hline C16 & -1.708 & 0.773 & -0.31 \\
\hline C17 & 0.85 & 0.923 & -0.449 \\
\hline C18 & 1.225 & 0.724 & -1.943 \\
\hline C19 & -1.961 & 2.159 & 0.382 \\
\hline $\mathrm{N} 2^{\prime}$ & -3.345 & 2.473 & 0.883 \\
\hline $\mathrm{N} 2^{\prime \prime}$ & 1.774 & 1.99 & -2.56 \\
\hline C22 & -3.765 & 1.601 & 2.041 \\
\hline C23 & -4.722 & 2.279 & 3.056 \\
\hline $\mathrm{N}^{2} \mathrm{~A}^{\prime}$ & -4.043 & 3.373 & 3.834 \\
\hline $\mathrm{C} 25$ & -4.981 & 4.417 & 4.383 \\
\hline C26 & -4.973 & 5.719 & 3.547 \\
\hline N4' & -5.511 & 5.466 & 2.186 \\
\hline C28 & -5.28 & 6.559 & 1.207 \\
\hline C29 & -5.266 & 5.97 & -0.22 \\
\hline N3B' & -3.999 & 5.196 & -0.481 \\
\hline C31 & -4.184 & 3.864 & -1.162 \\
\hline C32 & -4.398 & 2.665 & -0.182 \\
\hline C33 & 2.828 & 1.711 & -3.607 \\
\hline C34 & 4.207 & 1.217 & -3.067 \\
\hline N3A $A^{\prime \prime}$ & 4.893 & 2.196 & -2.15 \\
\hline C36 & 5.798 & 3.192 & -2.828 \\
\hline C37 & 5.571 & 4.62 & -2.286 \\
\hline $\mathrm{N} 4^{\prime \prime}$ & 4.27 & 5.134 & -2.783 \\
\hline C39 & 3.63 & 6.162 & -1.923 \\
\hline C40 & 2.148 & 6.354 & -2.323 \\
\hline N3B ${ }^{\prime \prime}$ & 1.32 & 5.114 & -2.095 \\
\hline C42 & 0.989 & 4.354 & -3.348 \\
\hline C43 & 0.647 & 2.862 & -3.073 \\
\hline H4 4 & -3.521 & 5.037 & 0.429 \\
\hline H45 & -3.363 & 3.847 & 3.205 \\
\hline H4 6 & -0.341 & -0.762 & -0.594 \\
\hline H47 & 2.596 & -0.094 & 4.049 \\
\hline H48 & 0.041 & -0.803 & 5.449 \\
\hline H49 & 0.236 & 2.425 & -4.012 \\
\hline H5O & 5.461 & 1.657 & -1.465 \\
\hline H51 & 4.162 & 2.723 & -1.629 \\
\hline
\end{tabular}




\begin{tabular}{|c|c|c|c|}
\hline H52 & 0.434 & 5.392 & -1.626 \\
\hline H53 & 4.884 & 0.991 & -3.916 \\
\hline H5 4 & 2.529 & -2.877 & 4.246 \\
\hline H55 & 2.485 & -3.388 & 2.536 \\
\hline H56 & 3.553 & -1.187 & 1.886 \\
\hline H57 & 4.539 & -2.084 & 3.08 \\
\hline H58 & 3.966 & 0.479 & 3.244 \\
\hline H59 & 5.241 & 0.267 & 4.975 \\
\hline $\mathrm{H} 60$ & 4.744 & -1.447 & 5.219 \\
\hline H61 & 3.028 & 0.993 & 6.077 \\
\hline H62 & 4.19 & 0.167 & 7.183 \\
\hline H63 & 3.004 & -1.811 & 7.047 \\
\hline H6 & 1.09 & 0.438 & 7.265 \\
\hline H65 & 1.795 & -0.592 & 8.564 \\
\hline H66 & -0.61 & -1.159 & 8.04 \\
\hline H67 & 0.47 & -2.562 & 7.737 \\
\hline H68 & -1.386 & -1.488 & 6.045 \\
\hline H69 & -0.816 & -3.617 & 5.574 \\
\hline H70 & 0.945 & -3.252 & 5.586 \\
\hline H71 & -1.082 & -2.3 & 3.468 \\
\hline H72 & -0.07 & -3.76 & 3.282 \\
\hline H73 & 0.176 & -2.521 & 1.14 \\
\hline H74 & 1.579 & -1.432 & 1.02 \\
\hline H75 & 0.094 & 0.447 & 2.062 \\
\hline H76 & -1.284 & -0.671 & 1.778 \\
\hline H77 & -2.499 & 0.05 & -0.03 \\
\hline H78 & -1.757 & 0.907 & -1.409 \\
\hline H79 & 1.726 & 0.619 & 0.16 \\
\hline $\mathrm{H} 8 \mathrm{O}$ & 0.649 & 1.987 & -0.225 \\
\hline H81 & 0.356 & 0.345 & -2.525 \\
\hline H82 & 1.987 & -0.09 & -1.955 \\
\hline H83 & -1.64 & 2.953 & -0.328 \\
\hline H8 4 & -1.267 & 2.233 & 1.243 \\
\hline H85 & -2.87 & 1.274 & 2.623 \\
\hline H8 6 & -4.25 & 0.675 & 1.656 \\
\hline H87 & -5.072 & 1.526 & 3.793 \\
\hline H8 8 & -5.602 & 2.697 & 2.527 \\
\hline H89 & -3.522 & 2.943 & 4.624 \\
\hline H9O & -4.624 & 4.647 & 5.409 \\
\hline H91 & -6.004 & 3.999 & 4.461 \\
\hline H92 & -3.925 & 6.092 & 3.487 \\
\hline H93 & -5.59 & 6.491 & 4.056 \\
\hline H94 & -6.52 & 5.322 & 2.265 \\
\hline H95 & -4.309 & 7.073 & 1.397 \\
\hline H96 & -6.092 & 7.313 & 1.298 \\
\hline H97 & -5.295 & 6.795 & -0.963 \\
\hline H98 & -6.147 & 5.317 & -0.38 \\
\hline H99 & -3.382 & 5.782 & -1.079 \\
\hline H100 & -3.27 & 3.689 & -1.766 \\
\hline H101 & -5.042 & 3.937 & -1.86 \\
\hline H102 & -5.39 & 2.827 & 0.298 \\
\hline H103 & -4.484 & 1.736 & -0.788 \\
\hline H10 & 3.042 & 2.635 & -4.191 \\
\hline H105 & 2.447 & 0.958 & -4.334 \\
\hline H106 & 4.072 & 0.282 & -2.485 \\
\hline H107 & 5.636 & 3.165 & -3.925 \\
\hline H108 & 6.84 & 2.875 & -2.616 \\
\hline H109 & 6.388 & 5.289 & -2.633 \\
\hline H110 & 5.596 & 4.591 & -1.172 \\
\hline H111 & 4.426 & 5.544 & -3.706 \\
\hline
\end{tabular}




\begin{tabular}{|l|l|l|l|}
\hline H112 & 4.172 & 7.127 & -2.03 \\
\hline H113 & 3.672 & 5.857 & -0.852 \\
\hline H114 & 2.06 & 6.678 & -3.379 \\
\hline H115 & 1.702 & 7.145 & -1.681 \\
\hline H116 & 1.85 & 4.483 & -1.461 \\
\hline H117 & 1.842 & 4.425 & -4.052 \\
\hline H118 & 0.109 & 4.852 & -3.806 \\
\hline H119 & -0.19 & 2.872 & -2.336 \\
\hline C120 & 2.01 & 3.301 & 1.873 \\
\hline C121 & -0.481 & 4.002 & 2.983 \\
\hline C122 & 1.258 & 4.366 & 1.347 \\
\hline C123 & 1.486 & 2.603 & 2.972 \\
\hline C124 & 0.229 & 2.914 & 3.522 \\
\hline C125 & -0.008 & 4.705 & 1.859 \\
\hline C126 & 3.208 & 2.893 & 1.268 \\
\hline 0127 & -0.815 & 5.653 & 1.213 \\
\hline 0128 & -0.331 & 2.104 & 4.519 \\
\hline 0129 & -0.46 & 6.231 & 0.147 \\
\hline 0130 & -1.972 & 5.94 & 1.629 \\
\hline C131 & 0.263 & 1.073 & 4.944 \\
\hline C132 & -1.472 & 2.323 & 5.015 \\
\hline 0133 & 3.662 & 3.461 & 0.236 \\
\hline 0134 & 3.876 & 1.902 & 1.684 \\
\hline H135 & -1.458 & 4.244 & 3.383 \\
\hline H136 & 1.643 & 4.907 & 0.493 \\
\hline H137 & 2.036 & 1.762 & 3.371 \\
\hline & & & \\
\hline
\end{tabular}


Table S9. Cartesian coordinates for the lowest energy conformer of the complex $\left[\mathrm{H}_{7} \mathbf{L} \mathbf{1 A}\right]^{4+}(\mathrm{A}=$ 1,3,5-BTC) with endo conformation of the bridgehead N1 nitrogen (a)

\begin{tabular}{|c|c|c|c|}
\hline atom & $\mathrm{x}$ & $y$ & $z$ \\
\hline $\mathrm{C} 1$ & 1.674 & -5.401 & 2.079 \\
\hline $\mathrm{C} 2$ & 2.486 & -6.727 & 2.15 \\
\hline N3A & 2.665 & -7.386 & 0.808 \\
\hline $\mathrm{C} 4$ & 3.018 & -8.852 & 0.896 \\
\hline $\mathrm{C5}$ & 1.828 & -9.784 & 0.556 \\
\hline N4 & 0.694 & -9.55 & 1.488 \\
\hline C7 & -0.62 & -10.066 & 1.03 \\
\hline $\mathrm{C} 8$ & -1.749 & -9.248 & 1.693 \\
\hline N3B & -1.862 & -7.877 & 1.077 \\
\hline C10 & -2.014 & -6.735 & 2.044 \\
\hline C11 & -0.658 & -6.159 & 2.563 \\
\hline N2 & 0.262 & -5.517 & 1.543 \\
\hline C13 & -0.303 & -4.201 & 1.052 \\
\hline C14 & 0.525 & -3.416 & -0.033 \\
\hline N1 & -0.229 & -2.683 & -1.161 \\
\hline C16 & -1.376 & -1.812 & -0.611 \\
\hline C17 & 0.728 & -1.873 & -2.057 \\
\hline C18 & 2.023 & -2.574 & -2.615 \\
\hline C19 & -2.182 & -0.897 & -1.609 \\
\hline $\mathrm{N} 2^{\prime}$ & -3.673 & -0.763 & -1.384 \\
\hline $\mathrm{N} 2^{\prime \prime}$ & 2.443 & -2.239 & -4.03 \\
\hline C22 & -4.029 & -0.295 & 0.012 \\
\hline C23 & -5.488 & -0.58 & 0.474 \\
\hline N3A' & -5.832 & -2.046 & 0.483 \\
\hline C25 & -7.317 & -2.319 & 0.474 \\
\hline C26 & -7.833 & -2.801 & -0.904 \\
\hline $\mathrm{N} 4^{\prime}$ & -7.586 & -1.773 & -1.949 \\
\hline C28 & -7.637 & -2.269 & -3.348 \\
\hline C29 & -6.752 & -1.373 & -4.241 \\
\hline N3B' & -5.289 & -1.651 & -4.008 \\
\hline C31 & -4.403 & -0.442 & -3.887 \\
\hline C32 & -4.305 & 0.126 & -2.437 \\
\hline C33 & 2.627 & -0.754 & -4.265 \\
\hline C34 & 2.588 & -0.29 & -5.751 \\
\hline N3A ${ }^{\prime \prime}$ & 1.287 & -0.61 & -6.439 \\
\hline C36 & 1.38 & -0.599 & -7.946 \\
\hline C37 & 1.384 & -2.02 & -8.56 \\
\hline $\mathrm{N} 4{ }^{\prime \prime}$ & 2.549 & -2.803 & -8.071 \\
\hline C39 & 2.438 & -4.274 & -8.239 \\
\hline C40 & 3.291 & -4.977 & -7.161 \\
\hline N3B ${ }^{\prime \prime}$ & 2.628 & -4.901 & -5.809 \\
\hline C42 & 3.528 & -4.549 & -4.657 \\
\hline C43 & 3.685 & -3.013 & -4.427 \\
\hline H4 4 & -4.94 & -2.231 & -4.797 \\
\hline H45 & -5.42 & -2.479 & 1.334 \\
\hline H4 6 & -0.662 & -3.413 & -1.76 \\
\hline H4 7 & 3.422 & -6.893 & 0.295 \\
\hline $\mathrm{H} 48$ & -2.686 & -7.875 & 0.443 \\
\hline H49 & 4.096 & -2.612 & -5.383 \\
\hline $\mathrm{H} 50$ & 0.575 & 0.087 & -6.145 \\
\hline H51 & 0.976 & -1.557 & -6.138 \\
\hline H52 & 2.193 & -5.825 & -5.61 \\
\hline H53 & 3.417 & -0.767 & -6.312 \\
\hline
\end{tabular}




\begin{tabular}{|c|c|c|c|}
\hline H5 4 & 2.281 & -4.722 & 1.437 \\
\hline H55 & 1.659 & -4.941 & 3.093 \\
\hline H5 6 & 1.981 & -7.437 & 2.835 \\
\hline H57 & 3.501 & -6.513 & 2.546 \\
\hline H58 & 1.778 & -7.295 & 0.27 \\
\hline H59 & 3.42 & -9.084 & 1.903 \\
\hline $\mathrm{H} 60$ & 3.828 & -9.022 & 0.155 \\
\hline $\mathrm{H} 61$ & 2.152 & -10.845 & 0.626 \\
\hline H62 & 1.518 & -9.584 & -0.496 \\
\hline H63 & 0.917 & -9.994 & 2.381 \\
\hline H6 4 & -0.714 & -11.137 & 1.313 \\
\hline H65 & -0.718 & -9.989 & -0.078 \\
\hline H66 & -1.575 & -9.157 & 2.784 \\
\hline H67 & -2.723 & -9.754 & 1.53 \\
\hline H68 & -1.006 & -7.707 & 0.509 \\
\hline H69 & -2.629 & -7.077 & 2.901 \\
\hline H70 & -2.583 & -5.943 & 1.515 \\
\hline H71 & -0.142 & -7.015 & 3.057 \\
\hline H72 & -0.887 & -5.428 & 3.372 \\
\hline H73 & -0.448 & -3.512 & 1.915 \\
\hline H7 4 & -1.306 & -4.396 & 0.613 \\
\hline H75 & 1.186 & -4.151 & -0.536 \\
\hline H76 & 1.156 & -2.665 & 0.483 \\
\hline H77 & -0.951 & -1.172 & 0.187 \\
\hline H78 & -2.102 & -2.516 & -0.154 \\
\hline H79 & 1.033 & -0.974 & -1.485 \\
\hline $\mathrm{H} 8 \mathrm{O}$ & 0.128 & -1.55 & -2.932 \\
\hline H81 & 1.846 & -3.67 & -2.555 \\
\hline H82 & 2.854 & -2.335 & -1.913 \\
\hline H83 & -2.019 & -1.311 & -2.628 \\
\hline H84 & -1.708 & 0.111 & -1.582 \\
\hline H85 & -3.835 & 0.798 & 0.107 \\
\hline H8 6 & -3.381 & -0.794 & 0.769 \\
\hline H87 & -6.199 & -0.048 & -0.19 \\
\hline H8 8 & -5.622 & -0.207 & 1.511 \\
\hline H89 & -5.412 & -2.491 & -0.359 \\
\hline H9O & -7.869 & -1.418 & 0.807 \\
\hline H91 & -7.49 & -3.123 & 1.221 \\
\hline H92 & -8.923 & -3.009 & -0.839 \\
\hline H93 & -7.312 & -3.753 & -1.157 \\
\hline H94 & -8.288 & -1.037 & -1.845 \\
\hline H95 & -8.689 & -2.246 & -3.709 \\
\hline H96 & -7.271 & -3.32 & -3.415 \\
\hline H97 & -6.966 & -0.301 & -4.051 \\
\hline H98 & -6.954 & -1.592 & -5.31 \\
\hline H99 & -5.202 & -2.202 & -3.129 \\
\hline H10O & -4.781 & 0.343 & -4.574 \\
\hline H101 & -3.398 & -0.746 & -4.244 \\
\hline H102 & -3.757 & 1.095 & -2.485 \\
\hline H103 & -5.351 & 0.377 & -2.146 \\
\hline H10 4 & 3.586 & -0.413 & -3.814 \\
\hline H105 & 1.82 & -0.176 & -3.759 \\
\hline H106 & 2.716 & 0.811 & -5.793 \\
\hline H107 & 2.276 & -0.031 & -8.265 \\
\hline H108 & 0.481 & -0.056 & -8.309 \\
\hline H109 & 1.428 & -1.945 & -9.668 \\
\hline H110 & 0.428 & -2.523 & -8.284 \\
\hline H111 & 3.381 & -2.486 & -8.574 \\
\hline H112 & 2.796 & -4.558 & -9.252 \\
\hline H113 & 1.38 & -4.612 & -8.138 \\
\hline
\end{tabular}




\begin{tabular}{|c|c|c|c|}
\hline H114 & 4.302 & -4.526 & -7.11 \\
\hline H115 & 3.394 & -6.055 & -7.408 \\
\hline H116 & 1.875 & -4.185 & -5.86 \\
\hline H117 & 4.523 & -5.006 & -4.833 \\
\hline H118 & 3.092 & -5.024 & -3.755 \\
\hline H119 & 4.48 & -2.862 & -3.661 \\
\hline C120 & -2.791 & -4.765 & -2.29 \\
\hline C121 & -0.375 & -5.072 & -3.695 \\
\hline C122 & -1.828 & -5.701 & -1.877 \\
\hline C123 & -2.522 & -4.005 & -3.442 \\
\hline C124 & -1.311 & -4.123 & -4.145 \\
\hline C125 & -0.602 & -5.853 & -2.549 \\
\hline C126 & -3.957 & -4.554 & -1.541 \\
\hline 0127 & 0.39 & -6.712 & -2.056 \\
\hline 0128 & -1.02 & -3.277 & -5.224 \\
\hline 0129 & 0.214 & -7.408 & -1.018 \\
\hline 0130 & 1.529 & -6.818 & -2.591 \\
\hline C131 & -1.806 & -2.364 & -5.607 \\
\hline C132 & 0.069 & -3.354 & -5.858 \\
\hline 0133 & -4.194 & -5.167 & -0.462 \\
\hline 0134 & -4.826 & -3.706 & -1.884 \\
\hline H135 & 0.568 & -5.17 & -4.214 \\
\hline H136 & -2.017 & -6.283 & -0.986 \\
\hline H137 & -3.245 & -3.272 & -3.771 \\
\hline
\end{tabular}


Description of the lowest energy conformer of the family with endo conformation of the N1 bridgehead nitrogen for the $\left[\mathrm{H}_{7} \mathrm{L1A}\right]^{4+}$ adduct $(\mathrm{A}=1,2,4-\mathrm{BTC})$ (Figure S8 b)

A full decription of the lowest energy conformer with exo conformation of the N1 bridgehead nitrogen for the $\left[\mathrm{H}_{7} \mathbf{L 1 A}\right]^{4+}$ adduct $(\mathrm{A}=1,2,4-\mathrm{BTC})$ (Figure $\mathrm{S} 8 \mathrm{a}$ ) is reported within the manuscript text. The lowest energy conformer of the family with endo conformation of the N1 bridgehead nitrogen (figure S8 b) has similar structural features to the complex with exo conformation of the bridgehead nitrogen (RMSD 1.8), one of the $\mathrm{N}_{4}$ unit being weakly interacting with the carboxylate anion. The most significant difference resides in the interaction mode of the carboxylate in 2 position, which gives rise also to an hydrogen bond contact with the protonated N1 bridgehead nitrogen (Figure S8 and Table S10). This conformer is $11.3 \mathrm{~kJ} \mathrm{~mol}^{-1}$ less stable than the former one, probably due to the weaker hydrogen bonding interaction observed between the carboxylate groups in 1 and 2 positions with the N2-N3A-N3B-N5 and N2'-N3A'-N3B'-N5' macrocyclic units. 
Table S10. Hydrogen bond interactions ( $\AA$, NH....O distance $\mathrm{d}<2.4 \AA$ ) for the lowest energy conformer of the family with exo conformation of the N1 bridgehead nitrogen (a) and for lowest energy conformer of the family with endo conformation of the N1 bridgehead nitrogen (b) for the $\left[\mathrm{H}_{7} \mathbf{L 1} \mathrm{A}\right]^{4+}$ adduct $(\mathrm{A}=1,2,4-\mathrm{BTC})$.

(a)

$\begin{array}{ll}\text { N3AH...O1 } & 1.96 \\ \text { N3BH...O1 } & 1.97 \\ \text { N3A'H...O5 } & 2.23 \\ \text { N4'H...O5 } & 2.09 \\ \text { N3A',H....O3 } & 1.94 \\ \text { N3B',H...O3 } & 2.11\end{array}$

(b)

$\begin{array}{ll}\text { N3AH.... 3 } & 1.97 \\ \text { N3BH....O6 } & 2.31 \\ \text { N1H....O3 } & 2.32 \\ \text { N3A'H...O6 } & 2.36 \\ \text { N3B'H....O5 } & 2.33 \\ \text { N3A' 'H....O1 } & 2.14 \\ \text { N3B' }{ }^{\prime} H \ldots . . . O 1 & 1.99\end{array}$

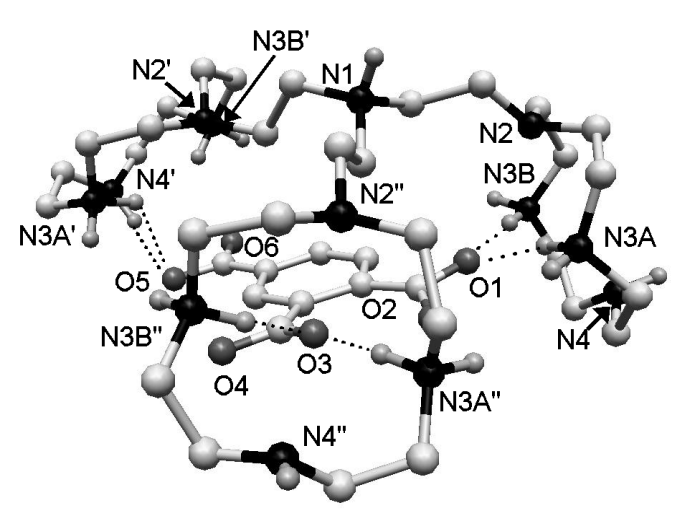

(a)

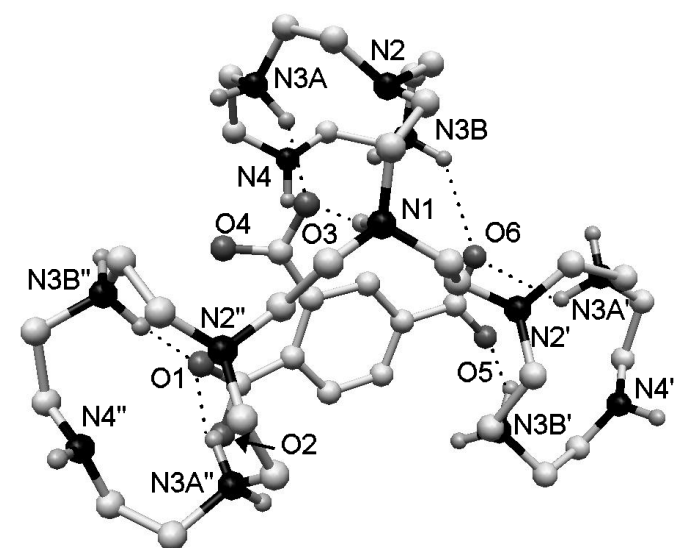

(b)

Figure S8. (a) Lowest energy conformer of the family with exo conformation of the N1 bridgehead nitrogen for the $\left[\mathrm{H}_{7} \mathbf{L 1 A}\right]^{4+}$ adduct $(\mathrm{A}=1,2,4-\mathrm{BTC})$. (b) Lowest energy conformer of the family with endo conformation of the N1 bridgehead nitrogen for the $\left[\mathrm{H}_{7} \mathbf{L} \mathbf{1 A}\right]^{4+}$ adduct $(\mathrm{A}=1,2,4-\mathrm{BTC})$. 
Table S11. Cartesian coordinates for the lowest energy conformer of the complex $\left[\mathrm{H}_{7} \mathbf{L} \mathbf{1 A}\right]^{4+}(\mathrm{A}=$ 1,2,4-BTC) with exo conformation of the N1 bridgehead nitrogen (a)

\begin{tabular}{|c|c|c|c|}
\hline atom & $x$ & $y$ & z \\
\hline $\mathrm{C} 1$ & -5.277 & 4.871 & 0.212 \\
\hline $\mathrm{C} 2$ & -5.427 & 4.412 & 1.697 \\
\hline N3A & -4.722 & 5.309 & 2.679 \\
\hline C4 & -5.556 & 6.421 & 3.261 \\
\hline C5 & -4.771 & 7.75 & 3.291 \\
\hline N4 & -4.667 & 8.286 & 1.91 \\
\hline C7 & -3.515 & 9.193 & 1.668 \\
\hline $\mathrm{C} 8$ & -3.213 & 9.292 & 0.153 \\
\hline N3B & -2.835 & 7.962 & -0.454 \\
\hline C10 & -3.911 & 7.358 & $-1 \cdot 314$ \\
\hline C11 & -3.729 & 5.828 & -1.535 \\
\hline N2 & -3.86 & 4.949 & -0.31 \\
\hline C13 & -3.263 & 3.582 & -0.553 \\
\hline C14 & -1.707 & 3.564 & -0.442 \\
\hline N1 & -1.163 & 2.339 & 0.289 \\
\hline C16 & 0.226 & 1.909 & -0.21 \\
\hline C17 & -1.271 & 2.449 & 1.809 \\
\hline C18 & -1.567 & 1.097 & 2.509 \\
\hline C19 & 1.403 & 2.929 & -0.007 \\
\hline N2' & 2.799 & 2.458 & -0.326 \\
\hline $\mathrm{N} 2^{\prime \prime}$ & -1.67 & 1.259 & 4.007 \\
\hline C22 & 3.295 & 1.396 & 0.625 \\
\hline C23 & 4.838 & 1.258 & 0.758 \\
\hline $\mathrm{N}^{2} \mathrm{~A}^{\prime}$ & 5.495 & 2.529 & 1.223 \\
\hline C25 & 6.974 & 2.613 & 0.931 \\
\hline C26 & 7.313 & 2.978 & -0.538 \\
\hline $\mathrm{N} 4^{\prime}$ & 7.039 & 4.414 & -0.832 \\
\hline $\mathrm{C} 28$ & 6.822 & 4.766 & -2.268 \\
\hline C29 & 5.585 & 4.13 & -2.972 \\
\hline N3B' & 4.282 & 4.41 & -2.274 \\
\hline C31 & 3.161 & 3.487 & -2.672 \\
\hline C32 & 3.072 & 2.205 & -1.789 \\
\hline C33 & -3.062 & 1.681 & 4.421 \\
\hline C34 & -3.206 & 2.298 & 5.844 \\
\hline N3A ${ }^{\prime \prime}$ & -2.479 & 3.605 & 5.995 \\
\hline C36 & -2.238 & 4.01 & 7.428 \\
\hline C37 & -0.758 & 3.845 & 7.848 \\
\hline $\mathrm{N}^{\prime \prime \prime}$ & -0.358 & 2.416 & 7.781 \\
\hline C39 & 1.104 & 2.171 & 7.705 \\
\hline C40 & 1.359 & 0.858 & 6.933 \\
\hline N3B' & 1.095 & 1.035 & 5.46 \\
\hline C42 & 0.389 & -0.11 & 4.786 \\
\hline C43 & -1.163 & 0.049 & 4.757 \\
\hline H4 4 & 3.999 & 5.389 & -2.483 \\
\hline H45 & 5.367 & 2.602 & 2.253 \\
\hline H4 6 & -1.799 & 1.558 & 0.025 \\
\hline H47 & -3.904 & 5.737 & 2.197 \\
\hline H48 & -2.62 & 7.3 & 0.321 \\
\hline H49 & -1.486 & 0.09 & 5.822 \\
\hline $\mathrm{H} 50$ & -3.032 & 4.352 & 5.529 \\
\hline H51 & -1.557 & 3.529 & 5.518 \\
\hline H52 & 2.007 & 1.187 & 4.983 \\
\hline
\end{tabular}




\begin{tabular}{|c|c|c|c|}
\hline H53 & -2.825 & 1.587 & 6.603 \\
\hline H5 4 & -5.764 & 5.871 & 0.155 \\
\hline H55 & -5.88 & 4.189 & -0.429 \\
\hline H5 6 & -4.994 & 3.399 & 1.825 \\
\hline H57 & -6.501 & 4.358 & 1.967 \\
\hline H58 & -4.371 & 4.723 & 3.463 \\
\hline H59 & -5.818 & 6.118 & 4.297 \\
\hline $\mathrm{H} 60$ & -6.49 & 6.537 & 2.676 \\
\hline $\mathrm{H} 61$ & -3.761 & 7.565 & 3.725 \\
\hline H62 & -5.296 & 8.488 & 3.935 \\
\hline H63 & -5.527 & 8.796 & 1.699 \\
\hline H6 4 & -2.601 & 8.814 & 2.18 \\
\hline $\mathrm{H} 65$ & -3.746 & 10.202 & 2.073 \\
\hline H66 & -2.34 & 9.962 & 0.004 \\
\hline $\mathrm{H} 67$ & -4.075 & 9.725 & -0.392 \\
\hline H68 & -1.982 & 8.092 & -1.032 \\
\hline H69 & -3.87 & 7.874 & -2.296 \\
\hline H70 & -4.897 & 7.558 & -0.85 \\
\hline H71 & -2.717 & 5.702 & -1.986 \\
\hline H72 & -4.462 & 5.501 & -2.307 \\
\hline H73 & -3.56 & 3.198 & -1.555 \\
\hline H74 & -3.66 & 2.852 & 0.189 \\
\hline H75 & -1.329 & 4.465 & 0.078 \\
\hline H76 & -1.298 & 3.563 & -1.473 \\
\hline H77 & 0.118 & 1.688 & -1.291 \\
\hline H78 & 0.467 & 0.957 & 0.303 \\
\hline H79 & -2.09 & 3.156 & 2.05 \\
\hline $\mathrm{H} 80$ & -0.331 & 2.878 & 2.201 \\
\hline H81 & -0.728 & 0.414 & 2.242 \\
\hline H82 & -2.495 & 0.645 & 2.091 \\
\hline H83 & 1.39 & 3.256 & 1.053 \\
\hline H84 & 1.179 & 3.836 & -0.61 \\
\hline H85 & 2.883 & 0.407 & 0.322 \\
\hline H86 & 2.912 & 1.604 & 1.653 \\
\hline H87 & 5.272 & 0.959 & -0.216 \\
\hline H8 8 & 5.074 & 0.475 & 1.509 \\
\hline H89 & 5.012 & 3.332 & 0.777 \\
\hline H9O & 7.434 & 1.639 & 1.197 \\
\hline H91 & 7.387 & 3.385 & 1.614 \\
\hline H92 & 6.727 & 2.312 & -1.208 \\
\hline H93 & 8.391 & 2.776 & -0.722 \\
\hline H94 & 6.222 & 4.717 & -0.299 \\
\hline H95 & 7.731 & 4.492 & -2.847 \\
\hline H96 & 6.72 & 5.873 & -2.331 \\
\hline H97 & 5.725 & 3.033 & -3.039 \\
\hline H98 & 5.501 & 4.543 & -3.999 \\
\hline H99 & 4.414 & 4.316 & -1.249 \\
\hline H100 & 3.286 & 3.206 & -3.737 \\
\hline H101 & 2.222 & 4.072 & -2.583 \\
\hline H102 & 2.29 & 1.535 & -2.21 \\
\hline H103 & 4.042 & 1.671 & -1.911 \\
\hline $\mathrm{H} 104$ & -3.751 & 0.809 & 4.352 \\
\hline H105 & -3.451 & 2.451 & 3.714 \\
\hline H106 & -4.279 & 2.496 & 6.048 \\
\hline H107 & -2.906 & 3.438 & 8.102 \\
\hline H108 & -2.512 & 5.084 & 7.499 \\
\hline H109 & -0.62 & 4.221 & 8.885 \\
\hline H110 & -0.128 & 4.463 & 7.166 \\
\hline H111 & -0.719 & 1.943 & 8.613 \\
\hline H112 & 1.519 & 2.095 & 8.733 \\
\hline
\end{tabular}




\begin{tabular}{|l|l|l|l|}
\hline H113 & 1.628 & 3.004 & 7.181 \\
\hline H114 & 0.72 & 0.045 & 7.333 \\
\hline H115 & 2.424 & 0.562 & 7.037 \\
\hline H116 & 0.514 & 1.89 & 5.337 \\
\hline H117 & 0.662 & -1.054 & 5.3 \\
\hline H118 & 0.783 & -0.16 & 3.751 \\
\hline H119 & -1.607 & -0.878 & 4.327 \\
\hline C120 & 0.227 & 5.812 & 2.318 \\
\hline C121 & 2.897 & 6.005 & 1.292 \\
\hline C122 & 1.298 & 5.138 & 2.989 \\
\hline C123 & 0.544 & 6.538 & 1.138 \\
\hline C124 & 1.834 & 6.628 & 0.636 \\
\hline C125 & 2.612 & 5.298 & 2.469 \\
\hline C126 & -0.233 & 7.054 & 0.585 \\
\hline 0127 & 2.004 & 7.189 & -0.277 \\
\hline 0128 & 3.442 & 4.805 & 2.966 \\
\hline 0129 & -1.151 & 5.807 & 2.663 \\
\hline 0130 & 1.204 & 4.28 & 4.115 \\
\hline C131 & 4.2 & 6.056 & 0.773 \\
\hline C132 & -2.041 & 6.262 & 1.888 \\
\hline 0133 & -1.675 & 5.387 & 3.726 \\
\hline 0134 & 0.146 & 3.843 & 4.633 \\
\hline H135 & 2.217 & 3.787 & 4.693 \\
\hline H136 & 5.171 & 5.482 & 1.338 \\
\hline H137 & 4.477 & 6.646 & -0.31 \\
\hline & & & \\
\hline
\end{tabular}


Table S12. Cartesian coordinates for the lowest energy conformer for the complex $\left[\mathrm{H}_{7} \mathbf{L} \mathbf{A} A\right]^{4+}(\mathrm{A}=$ 1,2,4-BTC) with endo conformation of the bridgehead N1 nitrogen (b)

\begin{tabular}{|c|c|c|c|}
\hline atom & $x$ & $\mathrm{Y}$ & $z$ \\
\hline $\mathrm{C} 1$ & -6.177 & -2.936 & -6.432 \\
\hline $\mathrm{C} 2$ & -6.482 & -4.292 & -7.137 \\
\hline N3A & -5.776 & -5.463 & -6.517 \\
\hline C4 & -5.775 & -6.694 & -7.386 \\
\hline C5 & -4.455 & -7.512 & -7.29 \\
\hline N4 & -3.208 & -6.721 & -7.495 \\
\hline C7 & -2.943 & -6.254 & -8.884 \\
\hline $\mathrm{C} 8$ & -2.003 & -5.018 & -8.889 \\
\hline N3B & -2.482 & -3.86 & -8.045 \\
\hline C10 & -3.606 & -3.072 & -8.652 \\
\hline C11 & -4.177 & -1.992 & -7.688 \\
\hline N2 & -4.739 & -2.455 & -6.353 \\
\hline C13 & -4.623 & -1.299 & -5.379 \\
\hline C14 & -4.806 & -1.586 & -3.847 \\
\hline N1 & -3.618 & -2.225 & -3.124 \\
\hline C16 & -2.371 & -1.338 & -3.076 \\
\hline C17 & -4.021 & -2.737 & -1.741 \\
\hline C18 & -3.408 & -4.138 & -1.464 \\
\hline C19 & -1.215 & -1.953 & -3.921 \\
\hline $\mathrm{N} 2^{\prime}$ & 0 & -1.098 & -4.186 \\
\hline $\mathrm{N}^{\prime \prime}{ }^{\prime}$ & -3.707 & -4.8 & -0.144 \\
\hline C22 & -0.292 & 0.039 & -5.134 \\
\hline C23 & 0.928 & 0.641 & -5.888 \\
\hline $\mathrm{N} \mathrm{A}^{\prime}$ & 1.578 & -0.329 & -6.837 \\
\hline C25 & 2.96 & 0.095 & -7.275 \\
\hline C26 & 4.087 & -0.743 & -6.622 \\
\hline $\mathrm{N} 4^{\prime}$ & 4.024 & -0.652 & -5.14 \\
\hline $\mathrm{C} 28$ & 4.684 & -1.762 & -4.407 \\
\hline C29 & 4.006 & -1.94 & -3.031 \\
\hline N3B' & 2.625 & -2.53 & -3.171 \\
\hline C31 & 1.569 & -1.919 & -2.29 \\
\hline C32 & 0.81 & -0.735 & -2.964 \\
\hline C33 & -3.009 & -4.212 & 1.054 \\
\hline C34 & -1.468 & -4.454 & 1.117 \\
\hline N3A ${ }^{\prime \prime}$ & -1.051 & -5.893 & 0.941 \\
\hline C36 & -1.184 & -6.762 & 2.166 \\
\hline C37 & -1.838 & -8.118 & 1.82 \\
\hline N4' ' & -3.271 & -7.912 & 1.487 \\
\hline C39 & -3.887 & -9.003 & 0.691 \\
\hline C40 & -5.217 & -8.515 & 0.074 \\
\hline N3B ${ }^{\prime \prime}$ & -4.985 & -7.485 & -1.004 \\
\hline C42 & -5.778 & -6.21 & -0.862 \\
\hline C43 & -5.148 & -5.164 & 0.112 \\
\hline H4 4 & 2.682 & -3.546 & -2.958 \\
\hline H45 & 0.978 & -0.423 & -7.68 \\
\hline H4 6 & -3.358 & -3.065 & -3.681 \\
\hline H4 7 & -6.245 & -5.695 & -5.619 \\
\hline $\mathrm{H} 48$ & -1.677 & -3.221 & -7.885 \\
\hline H49 & -5.246 & -5.597 & 1.134 \\
\hline $\mathrm{H} 50$ & -0.056 & -5.911 & 0.639 \\
\hline H51 & -1.633 & -6.309 & 0.185 \\
\hline H52 & -5.216 & -7.914 & -1.922 \\
\hline H53 & -1.067 & -4.092 & 2.085 \\
\hline
\end{tabular}




\begin{tabular}{|c|c|c|c|}
\hline H54 & -6.591 & -3.055 & -5.404 \\
\hline H55 & -6.8 & -2.157 & -6.93 \\
\hline H56 & -6.222 & -4.232 & -8.21 \\
\hline H57 & -7.572 & -4.494 & -7.056 \\
\hline H58 & -4.79 & -5.197 & -6.317 \\
\hline H59 & -5.944 & -6.408 & -8.444 \\
\hline $\mathrm{H} 60$ & -6.624 & -7.326 & -7.051 \\
\hline H61 & -4.49 & -8.353 & -8.018 \\
\hline H62 & -4.406 & -7.962 & -6.273 \\
\hline H63 & -2.419 & -7.313 & -7.226 \\
\hline H64 & -3.889 & -5.97 & -9.396 \\
\hline H65 & -2.474 & -7.079 & -9.464 \\
\hline H66 & -1.856 & -4.652 & -9.925 \\
\hline H67 & -1.011 & -5.31 & -8.483 \\
\hline H68 & -2.797 & -4.235 & -7.128 \\
\hline H69 & -4.397 & -3.771 & -8.976 \\
\hline H70 & -3.199 & -2.562 & -9.552 \\
\hline H71 & -4.95 & -1.41 & -8.241 \\
\hline H72 & -3.32 & -1.292 & -7.529 \\
\hline H73 & -5.363 & -0.51 & -5.651 \\
\hline H74 & -3.62 & -0.822 & -5.48 \\
\hline H75 & -5.678 & -2.255 & -3.71 \\
\hline H76 & -5.026 & -0.629 & -3.331 \\
\hline H77 & -2.027 & -1.232 & -2.029 \\
\hline H78 & -2.627 & -0.328 & -3.451 \\
\hline H79 & -5.125 & -2.83 & -1.692 \\
\hline $\mathrm{H} 80$ & -3.705 & -2.007 & -0.972 \\
\hline H81 & -2.306 & -4.083 & -1.605 \\
\hline H82 & -3.791 & -4.807 & -2.259 \\
\hline H83 & -1.635 & -2.28 & -4.9 \\
\hline H84 & -0.891 & -2.882 & -3.401 \\
\hline H85 & -0.793 & 0.863 & -4.576 \\
\hline H86 & -1.008 & -0.3 & -5.92 \\
\hline H87 & 1.686 & 0.991 & -5.16 \\
\hline H8 8 & 0.588 & 1.508 & -6.492 \\
\hline H89 & 1.65 & -1.256 & -6.372 \\
\hline H9O & 3.109 & 1.174 & -7.07 \\
\hline H91 & 2.998 & -0.053 & -8.375 \\
\hline H92 & 5.074 & -0.372 & -6.972 \\
\hline H93 & 3.975 & -1.798 & -6.962 \\
\hline H94 & 4.462 & 0.227 & -4.854 \\
\hline H95 & 5.762 & -1.526 & -4.273 \\
\hline H96 & 4.607 & -2.72 & -4.972 \\
\hline H97 & 3.946 & -0.966 & -2.504 \\
\hline H98 & 4.596 & -2.646 & -2.41 \\
\hline H99 & 2.325 & -2.417 & -4.161 \\
\hline $\mathrm{H} 100$ & 2.043 & -1.576 & -1.348 \\
\hline $\mathrm{H} 101$ & 0.858 & -2.731 & -2.037 \\
\hline $\mathrm{H} 102$ & 0.153 & -0.258 & -2.203 \\
\hline $\mathrm{H} 103$ & 1.584 & 0.021 & -3.229 \\
\hline $\mathrm{H} 104$ & -3.428 & -4.65 & 1.99 \\
\hline $\mathrm{H} 105$ & -3.2 & -3.117 & 1.102 \\
\hline H106 & -0.974 & -3.882 & 0.304 \\
\hline $\mathrm{H107}$ & -1.773 & -6.235 & 2.942 \\
\hline $\mathrm{H} 108$ & -0.157 & -6.933 & 2.552 \\
\hline H109 & -1.755 & -8.807 & 2.687 \\
\hline H110 & -1.292 & -8.571 & 0.96 \\
\hline H111 & -3.789 & -7.827 & 2.364 \\
\hline $\mathrm{H} 112$ & -4.076 & -9.881 & 1.346 \\
\hline $\mathrm{H} 113$ & -3.205 & -9.32 & -0.132 \\
\hline
\end{tabular}




\begin{tabular}{|c|c|c|c|}
\hline H114 & -5.877 & -8.098 & 0.861 \\
\hline H115 & -5.738 & -9.368 & -0.408 \\
\hline H116 & -3.974 & -7.237 & -1.002 \\
\hline H117 & -6.803 & -6.47 & -0.529 \\
\hline H118 & -5.85 & -5.77 & -1.878 \\
\hline H119 & -5.774 & -4.244 & 0.092 \\
\hline C120 & -0.502 & -6.477 & -3.215 \\
\hline C121 & 0.638 & -4.773 & -5.215 \\
\hline C122 & -1.348 & -5.851 & -4.183 \\
\hline C123 & 0.901 & -6.303 & -3.354 \\
\hline C124 & 1.457 & -5.472 & -4.322 \\
\hline C125 & -0.739 & -5.002 & -5.14 \\
\hline C126 & 1.583 & -6.782 & -2.661 \\
\hline 0127 & 2.534 & -5.347 & -4.352 \\
\hline 0128 & -1.369 & -4.472 & -5.84 \\
\hline 0129 & -0.918 & -7.242 & -2.099 \\
\hline 0130 & -2.759 & -5.922 & -4.266 \\
\hline C131 & 1.158 & -3.849 & -6.134 \\
\hline C132 & -2.093 & -7.308 & -1.656 \\
\hline 0133 & -0.109 & -7.874 & -1.358 \\
\hline 0134 & -3.437 & -5.049 & -4.887 \\
\hline H135 & -3.486 & -6.832 & -3.786 \\
\hline H136 & 0.424 & -3.197 & -6.933 \\
\hline H137 & 2.389 & -3.578 & -6.186 \\
\hline
\end{tabular}


Description of the lowest energy conformer of the family with exo conformation of the N1 bridgehead nitrogen for the $\left[\mathrm{H}_{7} \mathrm{L1A}\right]^{4+}$ adduct $(\mathrm{A}=1,2,3-\mathrm{BTC})$ (Figure S9 b)

A full decription of the lowest energy conformer with endo conformation of the N1 bridgehead nitrogen for the $\left[\mathrm{H}_{7} \mathbf{L 1 A}\right]^{4+}$ adduct $(\mathrm{A}=1,2,3-\mathrm{BTC})$ (Figure $\mathrm{S} 9$ a) is reported within the manuscript text. The lowest energy conformer with exo conformation of the N1 bridgehead nitrogen (Figure S9 b) is $13.7 \mathrm{~kJ} \mathrm{~mol}^{-1}$ less stable than the endo conformer. Although the conformation of the two complexes is not particularly different (RMSD 0.9), the exo conformer shows the presence of a lower number of hydrogen bonding contacts (Figure S9 and Table S13), leading to the observed lower stability. 
Table S13. Hydrogen bond interactions ( $\AA$, NH....O distance $d<2.4 \AA$ ) for the lowest energy conformer of the family with endo conformation of the N1 bridgehead nitrogen (a) and for lowest energy conformer of the family with exo conformation of the N1 bridgehead nitrogen (b) for the $\left[\mathrm{H}_{7} \mathbf{L 1} \mathrm{A}\right]^{4+}$ adduct $(\mathrm{A}=1,2,3-\mathrm{BTC})$.

(a)

$\begin{array}{ll}\text { N3AH....O6 } & 2.06 \\ \text { N3BH....O6 } & 2.05 \\ \text { N1H...O4 } & 2.12 \\ \text { N3A'H...O5 } & 2.32 \\ \text { N3A'H...O3 } & 2.32 \\ \text { N3A'H...O4 } & 2.19 \\ \text { N3B'H...O2 } & 2.27 \\ \text { N3B'H...O3 } & 2.23 \\ \text { N3A',H...O1 } & 2.14 \\ \text { N4',H....O1 } & 1.96 \\ \text { N3B',H...O1 } & 2.37\end{array}$

(b)

$\begin{array}{ll}\text { N3AH...O2 } & 2.08 \\ \text { N3BH...O4 } & 2.08 \\ \text { N3A'H...O5 } & 2.30 \\ \text { N3B'H...O5 } & 2.08 \\ \text { N3A',H...O1 } & 2.04 \\ \text { N3B',H...O1 } & 2.05 \\ & \\ & \end{array}$

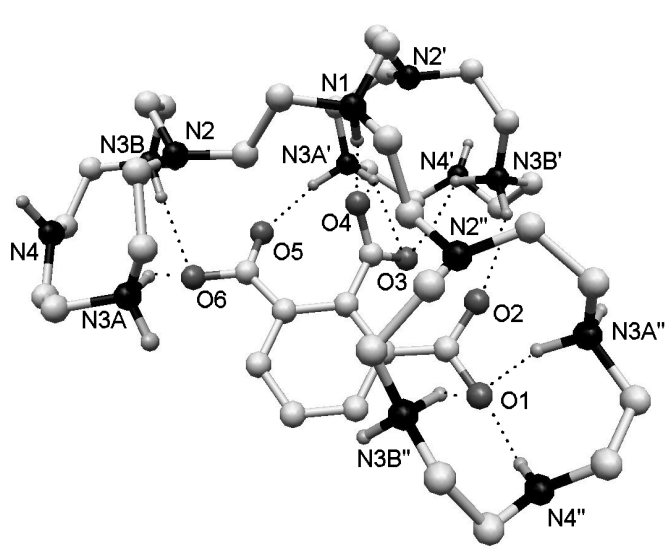

(a)

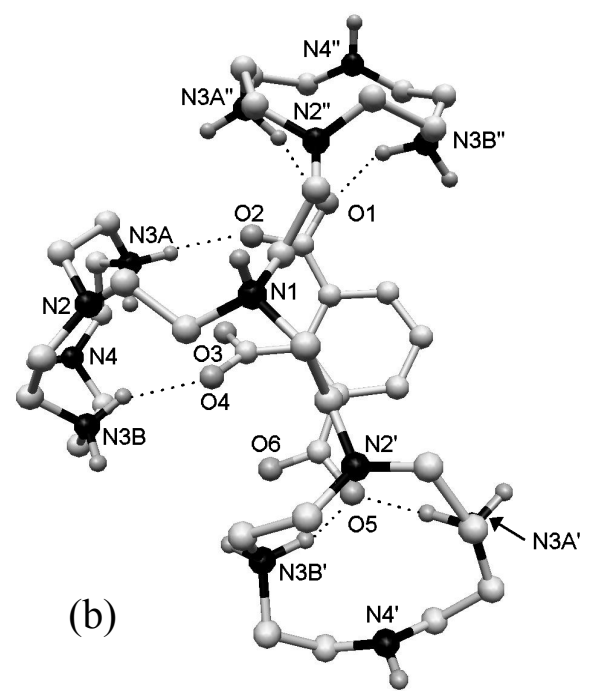

Figure S9. (a) Lowest energy conformer of the family with endo conformation of the N1 bridgehead nitrogen for the $\left[\mathrm{H}_{7} \mathbf{L 1} \mathrm{A}\right]^{4+}$ adduct $(\mathrm{A}=1,2,3-\mathrm{BTC})$. (b) Lowest energy conformer of the family with exo conformation of the N1 bridgehead nitrogen for the $\left[\mathrm{H}_{7} \mathbf{L} \mathbf{1 A}\right]^{4+}$ adduct $(\mathrm{A}=$ 1,2,3-BTC). 
Table S14. Cartesian coordinates for the lowest energy conformer for the complex $\left[\mathrm{H}_{7} \mathbf{L} \mathbf{A}\right]^{4+}(\mathrm{A}=$ 1,2,3-BTC) with endo conformation of the bridgehead nitrogen (a).

\begin{tabular}{|c|c|c|c|}
\hline atom & $x$ & y & $\mathrm{z}$ \\
\hline $\mathrm{C} 1$ & 7.12 & -5.677 & -4.541 \\
\hline $\mathrm{C} 2$ & 6.24 & -6.705 & -5.318 \\
\hline N3A & 5.633 & -7.78 & -4.453 \\
\hline C4 & 6.554 & -8.916 & -4.082 \\
\hline C5 & 6.484 & -9.227 & -2.571 \\
\hline N4 & 7.088 & -8.109 & -1.801 \\
\hline C7 & 6.711 & -8.065 & -0.365 \\
\hline $\mathrm{C} 8$ & 7.028 & -6.669 & 0.216 \\
\hline N3B & 6.118 & -5.61 & -0.355 \\
\hline C10 & 6.808 & -4.377 & -0.881 \\
\hline C11 & 7.409 & -4.537 & -2.314 \\
\hline N2 & 6.445 & -4.992 & -3.381 \\
\hline C13 & 5.356 & -4.014 & -3.744 \\
\hline C14 & 5.773 & -2.694 & -4.451 \\
\hline N1 & 4.55 & -1.826 & -4.745 \\
\hline C16 & 4.738 & -0.369 & -4.335 \\
\hline C17 & 4.039 & -1.975 & -6.178 \\
\hline C18 & 2.68 & -2.735 & -6.212 \\
\hline C19 & 4.542 & -0.108 & -2.807 \\
\hline N2' & 3.129 & -0.171 & -2.268 \\
\hline $\mathrm{N} 2^{\prime \prime}$ & 1.922 & -2.752 & -7.514 \\
\hline C22 & 3.136 & -0.204 & -0.756 \\
\hline C23 & 3.353 & -1.615 & -0.126 \\
\hline $\mathrm{N}^{2} \mathrm{~A}^{\prime}$ & 2.113 & -2.474 & -0.086 \\
\hline C25 & 1.114 & -2.136 & 0.995 \\
\hline C26 & -0.313 & -1.962 & 0.425 \\
\hline $\mathrm{N} 4^{\prime}$ & -0.372 & -0.759 & -0.446 \\
\hline $\mathrm{C} 28$ & -1.439 & -0.762 & -1.481 \\
\hline C29 & -0.993 & 0.085 & -2.696 \\
\hline N3B ${ }^{\prime}$ & 0.19 & -0.535 & -3.401 \\
\hline C31 & 1.23 & 0.436 & -3.896 \\
\hline C32 & 2.223 & 0.921 & -2.797 \\
\hline C33 & 1.355 & -1.408 & -7.896 \\
\hline C34 & 0.092 & -1.439 & -8.803 \\
\hline N3A ${ }^{\prime \prime}$ & -1.057 & -2.181 & -8.174 \\
\hline C36 & -2.151 & -2.574 & -9.139 \\
\hline C37 & -1.814 & -3.795 & -10.036 \\
\hline $\mathrm{N}^{\prime \prime \prime}$ & -1.839 & -5.072 & -9.268 \\
\hline C39 & -0.993 & -6.183 & -9.799 \\
\hline C40 & 0.548 & -5.953 & -9.793 \\
\hline N3B ${ }^{\prime \prime}$ & 1.106 & -5.624 & -8.435 \\
\hline C42 & 2.51 & -5.08 & -8.462 \\
\hline C43 & 2.571 & -3.532 & -8.631 \\
\hline H4 4 & -0.164 & -1.083 & -4.211 \\
\hline H45 & 2.409 & -3.463 & 0.044 \\
\hline H4 6 & 3.79 & -2.195 & -4.135 \\
\hline H47 & 4.816 & -8.179 & -4.958 \\
\hline H48 & 5.454 & -5.317 & 0.39 \\
\hline H49 & 2.076 & -3.299 & -9.601 \\
\hline $\mathrm{H} 50$ & -1.477 & -1.567 & -7.448 \\
\hline H51 & -0.688 & -3.031 & -7.707 \\
\hline H52 & 1.097 & -6.488 & -7.857 \\
\hline
\end{tabular}




\begin{tabular}{|c|c|c|c|}
\hline H53 & 0.342 & -1.914 & -9.772 \\
\hline H54 & 7.499 & -4.919 & -5.261 \\
\hline H55 & 8.008 & -6.246 & -4.18 \\
\hline H5 6 & 6.842 & -7.196 & -6.109 \\
\hline H57 & 5.395 & -6.177 & -5.806 \\
\hline H58 & 5.294 & -7.338 & -3.574 \\
\hline H59 & 7.594 & -8.671 & -4.377 \\
\hline $\mathrm{H} 60$ & 6.22 & -9.801 & -4.662 \\
\hline H61 & 7.036 & -10.168 & -2.353 \\
\hline H62 & 5.417 & -9.373 & -2.283 \\
\hline H63 & 8.104 & -8.197 & -1.86 \\
\hline H64 & 7.282 & -8.84 & 0.192 \\
\hline H65 & 5.623 & -8.27 & -0.237 \\
\hline H66 & 8.087 & -6.403 & 0.021 \\
\hline H67 & 6.862 & -6.676 & 1.313 \\
\hline H68 & 5.574 & -6.034 & -1.133 \\
\hline H69 & 7.606 & -4.092 & -0.166 \\
\hline H70 & 6.049 & -3.568 & -0.888 \\
\hline H71 & 8.24 & -5.274 & -2.218 \\
\hline H72 & 7.874 & -3.572 & -2.612 \\
\hline H73 & 4.814 & -3.734 & -2.814 \\
\hline H74 & 4.61 & -4.533 & -4.386 \\
\hline H75 & 6.311 & -2.904 & -5.394 \\
\hline H76 & 6.46 & -2.138 & -3.782 \\
\hline H77 & 4.019 & 0.257 & -4.897 \\
\hline H78 & 5.76 & -0.059 & -4.635 \\
\hline H79 & 4.783 & -2.526 & -6.784 \\
\hline $\mathrm{H} 80$ & 3.912 & -0.974 & -6.633 \\
\hline H81 & 2.021 & -2.292 & -5.432 \\
\hline H82 & 2.869 & -3.784 & -5.891 \\
\hline H83 & 4.975 & 0.894 & -2.583 \\
\hline H84 & 5.178 & -0.852 & -2.275 \\
\hline H85 & 2.171 & 0.178 & -0.35 \\
\hline H8 6 & 3.918 & 0.482 & -0.357 \\
\hline H87 & 3.731 & -1.511 & 0.911 \\
\hline H8 8 & 4.105 & -2.18 & -0.713 \\
\hline H89 & 1.635 & -2.397 & -1.006 \\
\hline H9O & 1.431 & -1.219 & 1.529 \\
\hline H91 & 1.123 & -2.984 & 1.713 \\
\hline H92 & -1.038 & -1.848 & 1.259 \\
\hline H93 & -0.581 & -2.882 & -0.144 \\
\hline H94 & -0.502 & 0.062 & 0.149 \\
\hline H95 & -2.373 & -0.342 & -1.049 \\
\hline H96 & -1.657 & -1.797 & -1.833 \\
\hline H97 & -0.736 & 1.112 & -2.368 \\
\hline H98 & -1.817 & 0.139 & -3.437 \\
\hline H99 & 0.651 & -1.19 & -2.739 \\
\hline $\mathrm{H} 100$ & 0.706 & 1.306 & -4.343 \\
\hline $\mathrm{H} 101$ & 1.785 & -0.082 & -4.703 \\
\hline $\mathrm{H} 102$ & 2.82 & 1.762 & -3.221 \\
\hline $\mathrm{H} 103$ & 1.603 & 1.374 & -1.988 \\
\hline $\mathrm{H} 104$ & 2.14 & -0.806 & -8.406 \\
\hline $\mathrm{H} 105$ & 1.062 & -0.847 & -6.977 \\
\hline $\mathrm{H106}$ & -0.251 & -0.401 & -8.991 \\
\hline $\mathrm{H107}$ & -2.382 & -1.692 & -9.772 \\
\hline $\mathrm{H} 108$ & -3.048 & -2.798 & -8.524 \\
\hline H109 & -0.819 & -3.625 & -10.503 \\
\hline $\mathrm{H} 110$ & -2.56 & -3.859 & -10.857 \\
\hline $\mathrm{H} 111$ & -1.555 & -4.886 & -8.305 \\
\hline $\mathrm{H} 112$ & -1.308 & -6.41 & -10.842 \\
\hline
\end{tabular}




\begin{tabular}{|c|c|c|c|}
\hline H113 & -1.207 & -7.091 & -9.194 \\
\hline H114 & 0.801 & -5.127 & -10.488 \\
\hline H115 & 1.054 & -6.878 & -10.14 \\
\hline H116 & 0.505 & -4.913 & -7.978 \\
\hline H117 & 3.071 & -5.575 & -9.281 \\
\hline H118 & 2.98 & -5.373 & -7.5 \\
\hline H119 & 3.635 & -3.227 & -8.741 \\
\hline C120 & 1.282 & -7.589 & -5.07 \\
\hline C121 & 0.365 & -6.612 & -5.415 \\
\hline C122 & 2.207 & -7.339 & -4.071 \\
\hline C123 & 2.222 & -6.122 & -3.353 \\
\hline C124 & 0.373 & -5.333 & -4.815 \\
\hline C125 & -0.457 & -4.354 & -5.43 \\
\hline C126 & 3.102 & -6.074 & -2.238 \\
\hline 0127 & -1.234 & -4.628 & -6.389 \\
\hline 0128 & -0.473 & -3.12 & -5.163 \\
\hline 0129 & 3.982 & -6.959 & -2.03 \\
\hline 0130 & 3.108 & -5.186 & -1.342 \\
\hline C131 & 1.292 & -5.096 & -3.736 \\
\hline C132 & 1.288 & -3.861 & -3.072 \\
\hline 0133 & 0.234 & -3.377 & -2.579 \\
\hline 0134 & 2.329 & -3.16 & -2.943 \\
\hline H135 & 2.876 & -8.147 & -3.799 \\
\hline H136 & 1.27 & -8.549 & -5.573 \\
\hline H137 & -0.329 & -6.839 & -6.218 \\
\hline
\end{tabular}


Table S15. Cartesian coordinates for the lowest energy conformer for the complex $\left[\mathrm{H}_{7} \mathbf{L} 1 \mathrm{~A}\right]^{4+}(\mathrm{A}=$ 1,2,3-BTC) with exo conformation of the bridgehead N1 nitrogen (b)

\begin{tabular}{|c|c|c|c|}
\hline atom & $x$ & y & $\mathrm{z}$ \\
\hline $\mathrm{C} 1$ & -0.877 & 3.541 & -1.724 \\
\hline $\mathrm{C} 2$ & 0.526 & 4.178 & -1.496 \\
\hline N3A & 1.416 & 3.342 & -0.617 \\
\hline C4 & 2.3 & 2.366 & -1.347 \\
\hline $\mathrm{C5}$ & 2.563 & 1.101 & -0.499 \\
\hline N4 & 1.311 & 0.306 & -0.385 \\
\hline C7 & 0.983 & -0.179 & 0.981 \\
\hline $\mathrm{C} 8$ & -0.517 & -0.545 & 1.082 \\
\hline N3B & -1.415 & 0.664 & 1.011 \\
\hline C10 & -2.063 & 0.862 & -0.333 \\
\hline C11 & -2.69 & 2.275 & -0.505 \\
\hline $\mathrm{N} 2$ & -1.711 & 3.426 & -0.471 \\
\hline C13 & -2.374 & 4.728 & -0.092 \\
\hline C14 & -2.61 & 4.822 & 1.445 \\
\hline N1 & -2.492 & 6.219 & 2.056 \\
\hline C16 & -3.183 & 6.295 & 3.428 \\
\hline C17 & -1.07 & 6.781 & 2.061 \\
\hline C18 & -1.034 & 8.307 & 1.773 \\
\hline C19 & -2.564 & 5.411 & 4.569 \\
\hline $\mathrm{N} 2^{\prime}$ & -3.453 & 4.972 & 5.7 \\
\hline $\mathrm{N} 2^{\prime \prime}$ & 0.276 & 8.781 & 1.184 \\
\hline C22 & -3.774 & 6.079 & 6.67 \\
\hline C23 & -3.924 & 5.614 & 8.144 \\
\hline $\mathrm{N} \mathrm{A}^{\prime}$ & -2.65 & 5.038 & 8.704 \\
\hline C25 & -2.859 & 4.148 & 9.904 \\
\hline C26 & -2.677 & 2.647 & 9.574 \\
\hline $\mathrm{N} 4^{\prime}$ & -3.674 & 2.207 & 8.565 \\
\hline $\mathrm{C} 28$ & -3.349 & 0.934 & 7.872 \\
\hline C29 & -4.039 & 0.914 & 6.491 \\
\hline N3B' & -3.354 & 1.85 & 5.53 \\
\hline C31 & -4.267 & 2.703 & 4.691 \\
\hline C32 & -4.618 & 4.077 & 5.344 \\
\hline C33 & 0.295 & 8.547 & -0.312 \\
\hline C34 & 1.651 & 8.729 & -1.057 \\
\hline N3A ${ }^{\prime \prime}$ & 2.712 & 7.759 & -0.618 \\
\hline C36 & 4.11 & 8.171 & -1.015 \\
\hline C37 & 4.961 & 8.644 & 0.188 \\
\hline $\mathrm{N}^{\prime}{ }^{\prime}$ & 4.326 & 9.81 & 0.856 \\
\hline C39 & 4.734 & 10.031 & 2.267 \\
\hline C40 & 3.598 & 10.756 & 3.021 \\
\hline N3B' & 2.433 & 9.832 & 3.277 \\
\hline C42 & 1.072 & 10.422 & 3.026 \\
\hline $\mathrm{C} 43$ & 0.567 & 10.216 & 1.564 \\
\hline $\mathrm{H} 44$ & -2.756 & 1.285 & 4.894 \\
\hline H45 & -2.023 & 5.821 & 8.976 \\
\hline H4 6 & -3.039 & 6.846 & 1.432 \\
\hline H47 & 0.813 & 2.805 & 0.037 \\
\hline H48 & -0.85 & 1.507 & 1.24 \\
\hline H49 & 1.359 & 10.641 & 0.906 \\
\hline $\mathrm{H} 50$ & 2.509 & 6.83 & -1.037 \\
\hline H51 & 2.678 & 7.673 & 0.419 \\
\hline H52 & 2.477 & 9.523 & 4.269 \\
\hline H53 & 2.026 & 9.761 & -0.91 \\
\hline
\end{tabular}




\begin{tabular}{|c|c|c|c|}
\hline H5 4 & -0.697 & 2.536 & -2.17 \\
\hline H55 & -1.42 & 4.133 & -2.495 \\
\hline H5 6 & 0.418 & 5.161 & -0.993 \\
\hline H57 & 1.031 & 4.343 & -2.469 \\
\hline H58 & 2.02 & 3.979 & -0.057 \\
\hline H59 & 3.258 & 2.886 & -1.555 \\
\hline $\mathrm{H} 60$ & 1.824 & 2.085 & -2.309 \\
\hline H61 & 2.96 & 1.404 & 0.498 \\
\hline H62 & 3.339 & 0.479 & -0.995 \\
\hline H63 & 1.395 & -0.507 & -0.998 \\
\hline H6 4 & 1.204 & 0.603 & 1.741 \\
\hline H65 & 1.604 & -1.071 & 1.214 \\
\hline H66 & -0.703 & -1.013 & 2.072 \\
\hline H67 & -0.804 & -1.275 & 0.298 \\
\hline H68 & -2.163 & 0.559 & 1.724 \\
\hline H69 & -2.861 & 0.096 & -0.428 \\
\hline H70 & -1.306 & 0.688 & -1.124 \\
\hline H71 & -3.44 & 2.381 & 0.314 \\
\hline H72 & -3.261 & 2.295 & -1.461 \\
\hline H73 & -3.345 & 4.844 & -0.624 \\
\hline H74 & -1.739 & 5.589 & -0.398 \\
\hline H75 & -1.898 & 4.17 & 1.984 \\
\hline H76 & -3.638 & 4.454 & 1.646 \\
\hline H77 & -4.244 & 6.023 & 3.263 \\
\hline H78 & -3.157 & 7.357 & 3.743 \\
\hline H79 & -0.474 & 6.243 & 1.299 \\
\hline $\mathrm{H} 8 \mathrm{O}$ & -0.607 & 6.579 & 3.045 \\
\hline H81 & -1.222 & 8.795 & 2.758 \\
\hline H82 & -1.885 & 8.597 & 1.114 \\
\hline H83 & -1.703 & 5.978 & 4.989 \\
\hline H84 & -2.123 & 4.498 & 4.116 \\
\hline H85 & -4.709 & 6.595 & 6.357 \\
\hline H8 6 & -2.966 & 6.849 & 6.664 \\
\hline H87 & -4.726 & 4.852 & 8.214 \\
\hline H8 8 & -4.197 & 6.482 & 8.779 \\
\hline H89 & -2.186 & 4.478 & 7.959 \\
\hline H9O & -3.858 & 4.335 & 10.345 \\
\hline H91 & -2.093 & 4.445 & 10.651 \\
\hline H92 & -2.8 & 2.046 & 10.502 \\
\hline H93 & -1.64 & 2.494 & 9.196 \\
\hline H94 & -4.575 & 2.092 & 9.036 \\
\hline H95 & -3.701 & 0.078 & 8.487 \\
\hline H96 & -2.248 & 0.827 & 7.726 \\
\hline H97 & -5.108 & 1.189 & 6.589 \\
\hline H98 & -3.97 & -0.104 & 6.054 \\
\hline H99 & -2.739 & 2.483 & 6.081 \\
\hline H10O & -5.195 & 2.136 & 4.482 \\
\hline H101 & -3.744 & 2.872 & 3.727 \\
\hline H102 & -5.306 & 4.62 & 4.659 \\
\hline H103 & -5.202 & 3.845 & 6.263 \\
\hline H1O 4 & -0.446 & 9.223 & -0.8 \\
\hline H105 & -0.035 & 7.504 & -0.53 \\
\hline H106 & 1.49 & 8.564 & -2.144 \\
\hline H107 & 4.068 & 8.957 & -1.796 \\
\hline H108 & 4.586 & 7.271 & -1.459 \\
\hline H109 & 5.977 & 8.928 & -0.163 \\
\hline H110 & 5.066 & 7.79 & 0.896 \\
\hline H111 & 4.556 & 10.651 & 0.32 \\
\hline H112 & 5.66 & 10.646 & 2.292 \\
\hline H113 & 4.946 & 9.064 & 2.781 \\
\hline
\end{tabular}




\begin{tabular}{|l|l|l|l|}
\hline H114 & 3.257 & 11.641 & 2.445 \\
\hline H115 & 3.963 & 11.095 & 4.012 \\
\hline H116 & 2.542 & 8.998 & 2.666 \\
\hline H117 & 1.102 & 11.505 & 3.265 \\
\hline H118 & 0.379 & 9.935 & 3.741 \\
\hline H119 & -0.339 & 10.845 & 1.409 \\
\hline C120 & 1.512 & 6.276 & 6.035 \\
\hline C121 & 2.12 & 6.452 & 4.806 \\
\hline C122 & 0.709 & 5.172 & 6.247 \\
\hline C123 & 0.529 & 4.171 & 5.263 \\
\hline C124 & 1.914 & 5.553 & 3.737 \\
\hline C125 & 2.362 & 6.01 & 2.467 \\
\hline C126 & -0.203 & 3.032 & 5.696 \\
\hline 0127 & 3.069 & 7.049 & 2.325 \\
\hline 0128 & 2.051 & 5.509 & 1.352 \\
\hline 0129 & -0.854 & 3.019 & 6.782 \\
\hline 0130 & -0.292 & 1.931 & 5.089 \\
\hline C131 & 1.152 & 4.355 & 3.98 \\
\hline C132 & 1.037 & 3.383 & 2.964 \\
\hline 0133 & 2.033 & 3.014 & 2.282 \\
\hline 0134 & -0.049 & 2.826 & 2.641 \\
\hline H135 & 0.266 & 5.064 & 7.231 \\
\hline H136 & 1.657 & 7.005 & 6.824 \\
\hline H137 & 2.704 & 7.354 & 4.665 \\
\hline & & & \\
\hline & & & \\
\hline 0136 & & & \\
\hline
\end{tabular}




\section{Description of the lowest energy conformer for the $\left[\mathrm{H}_{9} \mathrm{~L} 2 \mathrm{~A}\right]^{6+}$ adducts $(\mathrm{A}=1,3,5-\mathrm{BTC}, 1,2,3-$ BTC and 1,2,4-BTC)}

Considering the nonaprotonated receptor $\mathbf{L 2}$, the 1,3,5- and the 1,2,4-BTC trianions give rise to complexes with conformations similar to those found with L1. In both cases, the lowest energy conformers show an exo disposition of the bridgehead nitrogen N1.

Similarly to the $\left[\mathrm{H}_{7} \mathbf{L} \mathbf{1}(1,3,5-\mathrm{BTC})\right]^{4+}$ adduct, the lowest energy conformer of the $\left[\mathrm{H}_{9} \mathbf{L} 2(1,3,5-\right.$ BTC) $]^{6+}$ adduct shows the anion encapsulated inside the receptor cavity (Figure S10) with an approximated $\mathrm{C}_{3 \mathrm{v}}$ symmetry; the aromatic ring of the substrate is almost perpendicular to the $\mathrm{C}_{3 \mathrm{v}}$ axis passing through the bridgehead nitrogen $\mathrm{N} 1$ and the centroid of the ring.

In comparison with the $\mathbf{L} \mathbf{1}$ complex, however, a different hydrogen bonding array is observed. Each N4 cyclic unit, in fact, interacts with a single oxygen of a carboxylate function, with the only exception of the O3 $\cdots$ N3B” hydrogen bond (Figure S10).

A similar interaction involving each carboxylate group and a macrocyclic unit is present in the 1,2,4-BTC complex (Figure S11), where the two N4 units N2'-N3A'-N3B'-N4' and N2-N3A-N3BN4 interacting, respectively, with the carboxylate in 1 and 2 positions are close to each other (N4 'N N4' $9.7 \AA$ ), while the remaining N2"-N3A"-N3B"-N4" unit is located at a slightly longer

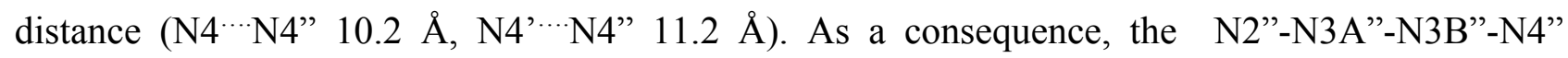
macrocyclic unit gives rise to a weaker interaction with the carboxylate in 4 position.

Differently from the 1,3,5-BTC and 1,2,4-BTC adducts with $\mathbf{L 1}$ and $\mathbf{L 2}$, the $\left[\mathrm{H}_{9} \mathbf{L 2}(1,2,3-\mathrm{BTC})\right]^{6+}$ complex displays a remarkably different conformation with respect to the $\left[\mathrm{H}_{7} \mathbf{L} \mathbf{1}(1,2,3-\mathrm{BTC})\right]^{4+}$ one, the N1 nitrogen being in exo-conformation. As shown in Figure S12, two macrocyclic units, N2'N3A'-N3B'-N4' and N2-N3A-N3B-N4 are close to each other (N4'…N4 $9.2 \AA$ ) and originate a hydrogen bond network involving all three carboxylate groups, while the N2"-N3A"-N3B"-N4" moiety lies at a longer distance (N4 “.N 4 ” $11.4 \AA$, N4 ${ }^{\prime \cdots}$ N4” $12.6 \AA$ ) and does not participate in 
substrate binding. 
Table S16. Hydrogen bond interactions ( $\AA$, NH....O distance $d<2.4 \AA$ ) for the lowest energy conformer of the $\left[\mathrm{H}_{9} \mathbf{L} 2 \mathrm{~A}\right]^{6+}$ adduct $(\mathrm{A}=1,3,5-\mathrm{BTC})$.

\begin{tabular}{|c|c|}
\hline N3AH....O4 & 2.21 \\
\hline N4H....O4 & 1.97 \\
\hline N3BH....O4 & 1.95 \\
\hline N3A'H....O1 & 1.93 \\
\hline N4'H....O1 & 1.99 \\
\hline N3B'H....O1 & 2.02 \\
\hline N3A'”H....O6 & 2.11 \\
\hline N4'’H....O6 & 2.08 \\
\hline N3B'”H....O5 & 2.18 \\
\hline
\end{tabular}

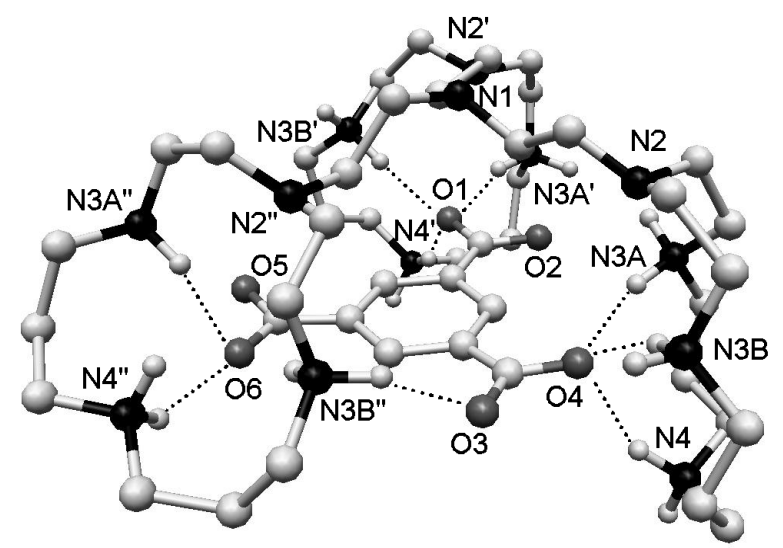

Figure S10. Lowest energy conformer of the $\left[\mathrm{H}_{9} \mathbf{L} 2 \mathrm{~A}\right]^{6+}$ adduct $(\mathrm{A}=1,3,5-\mathrm{BTC})$. 
Table S17. Cartesian coordinates for the lowest energy conformer of the complex $\left[\mathrm{H}_{9} \mathbf{L} 2 \mathrm{~A}\right]^{6+}(\mathrm{A}=$ 1,3,5-BTC)

\begin{tabular}{|c|c|c|c|}
\hline atom & $\mathrm{x}$ & $\mathrm{y}$ & $z$ \\
\hline N1 & 2.699 & -1.725 & -2.079 \\
\hline $\mathrm{C} 2$ & 4.087 & -1.741 & -1.483 \\
\hline C3 & 4.381 & -0.574 & -0.49 \\
\hline N2 & 5.796 & -0.583 & 0.033 \\
\hline C5 & 6.785 & -0.037 & -0.976 \\
\hline C6 & 8.103 & -0.85 & -1.078 \\
\hline N3A & 7.893 & -2.227 & -1.644 \\
\hline $\mathrm{C} 8$ & 9.171 & -2.962 & -1.956 \\
\hline C9 & 9.454 & -4.128 & -0.975 \\
\hline C10 & 10.062 & -3.731 & 0.392 \\
\hline N4 & 9.494 & -4.561 & 1.509 \\
\hline C12 & 9.886 & -4.093 & 2.885 \\
\hline C13 & 8.719 & -3.409 & 3.644 \\
\hline C14 & 8.523 & -1.895 & 3.375 \\
\hline N3B & 7.24 & -1.61 & 2.65 \\
\hline C16 & 7.111 & -0.17 & 2.256 \\
\hline C17 & 5.852 & 0.097 & 1.382 \\
\hline C18 & 2.58 & -2.476 & -3.38 \\
\hline C19 & 3.202 & -1.743 & -4.611 \\
\hline N2' & 3.353 & -2.633 & -5.819 \\
\hline C21 & 4.43 & -2.092 & -6.733 \\
\hline $\mathrm{C} 22$ & 5.044 & -3.095 & -7.75 \\
\hline N3A' & 5.754 & -4.226 & -7.07 \\
\hline C24 & 6.094 & -5.347 & -8.009 \\
\hline $\mathrm{C} 25$ & 6.677 & -6.575 & -7.264 \\
\hline $\mathrm{C} 26$ & 5.885 & -7.9 & -7.416 \\
\hline N4' & 4.756 & -8.022 & -6.427 \\
\hline C28 & 3.388 & -7.858 & -7.027 \\
\hline C29 & 2.296 & -7.773 & -5.931 \\
\hline C30 & 1.203 & -6.696 & -6.144 \\
\hline $\mathrm{N}^{\prime} \mathrm{B}^{\prime}$ & 1.659 & -5.309 & -5.766 \\
\hline C32 & 1.84 & -4.387 & -6.94 \\
\hline C33 & 2.035 & -2.907 & -6.514 \\
\hline C34 & 1.58 & -2.052 & -1.116 \\
\hline C35 & 1.548 & -3.522 & -0.562 \\
\hline $\mathrm{N} 2^{\prime \prime}$ & 0.787 & -3.785 & 0.71 \\
\hline C37 & -0.714 & -3.667 & 0.614 \\
\hline C38 & -1.419 & -4.725 & -0.289 \\
\hline N3A ${ }^{\prime \prime}$ & -1.178 & -6.147 & 0.14 \\
\hline C40 & -2.201 & -6.671 & 1.111 \\
\hline C41 & -1.841 & -8.086 & 1.631 \\
\hline C42 & -1.21 & -8.157 & 3.044 \\
\hline N4' & 0.197 & -7.649 & 3.049 \\
\hline C44 & 0.904 & -7.843 & 4.354 \\
\hline C45 & 2.36 & -7.319 & 4.296 \\
\hline C46 & 2.545 & -5.793 & 4.496 \\
\hline N3B' ${ }^{\prime}$ & 2.434 & -5.023 & 3.209 \\
\hline C48 & 1.338 & -4 & 3.198 \\
\hline C49 & 1.386 & -3.122 & 1.923 \\
\hline H5O & 4.266 & -2.699 & -0.954 \\
\hline H51 & 4.846 & -1.677 & -2.294 \\
\hline H52 & 3.66 & -0.704 & 0.351 \\
\hline
\end{tabular}




\begin{tabular}{|c|c|c|c|}
\hline H53 & 4.148 & 0.399 & -0.977 \\
\hline H5 4 & 7.04 & 1.021 & -0.739 \\
\hline H55 & 6.348 & -0.02 & -2.003 \\
\hline H56 & 8.577 & -0.962 & -0.084 \\
\hline H57 & 8.8 & -0.307 & -1.751 \\
\hline H58 & 7.338 & -2.144 & -2.52 \\
\hline H59 & 7.352 & -2.787 & -0.955 \\
\hline $\mathrm{H} 60$ & 10.022 & -2.253 & -1.99 \\
\hline H61 & 9.04 & -3.385 & -2.976 \\
\hline H62 & 10.169 & -4.821 & -1.475 \\
\hline H63 & 8.512 & -4.7 & -0.829 \\
\hline H64 & 9.86 & -2.666 & 0.626 \\
\hline H65 & 11.16 & -3.896 & 0.377 \\
\hline H66 & 8.456 & -4.535 & 1.439 \\
\hline H67 & 10.773 & -3.432 & 2.824 \\
\hline H68 & 10.184 & -5.005 & 3.447 \\
\hline H69 & 8.926 & -3.524 & 4.734 \\
\hline H70 & 7.778 & -3.967 & 3.446 \\
\hline H71 & 9.36 & -1.481 & 2.775 \\
\hline H72 & 8.481 & -1.357 & 4.346 \\
\hline H73 & 6.443 & -1.863 & 3.269 \\
\hline H7 4 & 7.2 & -2.199 & 1.794 \\
\hline H75 & 8.026 & 0.138 & 1.714 \\
\hline H76 & 7.031 & 0.426 & 3.19 \\
\hline H77 & 5.755 & 1.199 & 1.248 \\
\hline H78 & 4.982 & -0.225 & 2.003 \\
\hline H79 & 3.035 & -3.484 & -3.277 \\
\hline $\mathrm{H} 8 \mathrm{O}$ & 1.504 & -2.629 & -3.627 \\
\hline H81 & 2.582 & -0.853 & -4.858 \\
\hline H82 & 4.2 & -1.368 & -4.29 \\
\hline H83 & 5.289 & -1.716 & -6.126 \\
\hline H8 4 & 4.036 & -1.218 & -7.299 \\
\hline H85 & 5.791 & -2.564 & -8.377 \\
\hline H8 6 & 4.252 & -3.504 & -8.408 \\
\hline H87 & 5.149 & -4.597 & -6.309 \\
\hline H8 8 & 6.634 & -3.865 & -6.651 \\
\hline H89 & 6.85 & -4.961 & -8.726 \\
\hline H9O & 5.176 & -5.617 & -8.571 \\
\hline H91 & 6.812 & -6.354 & -6.182 \\
\hline H92 & 7.698 & -6.755 & -7.675 \\
\hline H93 & 6.571 & -8.749 & -7.208 \\
\hline H94 & 5.501 & -8.024 & -8.448 \\
\hline H95 & 4.809 & -8.957 & -5.975 \\
\hline H96 & 3.2 & -8.738 & -7.677 \\
\hline H97 & 3.399 & -6.943 & -7.654 \\
\hline H98 & 2.759 & -7.61 & -4.934 \\
\hline H99 & 1.786 & -8.763 & -5.884 \\
\hline H100 & 0.344 & -6.929 & -5.478 \\
\hline H101 & 0.833 & -6.707 & -7.188 \\
\hline H102 & 2.568 & -5.387 & -5.266 \\
\hline H103 & 0.956 & -4.893 & -5.124 \\
\hline H10 4 & 0.927 & -4.451 & -7.57 \\
\hline H105 & 2.707 & -4.751 & -7.523 \\
\hline H106 & 1.17 & -2.652 & -5.858 \\
\hline H107 & 1.928 & -2.263 & -7.417 \\
\hline H108 & 1.627 & -1.348 & -0.257 \\
\hline H109 & 0.605 & -1.861 & -1.616 \\
\hline H110 & 1.155 & -4.178 & -1.369 \\
\hline H111 & 2.595 & -3.844 & -0.396 \\
\hline H112 & -0.983 & -2.653 & 0.243 \\
\hline
\end{tabular}




\begin{tabular}{|c|c|c|c|}
\hline H113 & -1.163 & -3.766 & 1.629 \\
\hline H114 & -1.04 & -4.639 & -1.328 \\
\hline H115 & -2.511 & -4.532 & -0.311 \\
\hline H116 & -0.235 & -6.208 & 0.573 \\
\hline H117 & -1.191 & -6.751 & -0.707 \\
\hline H118 & -3.172 & -6.716 & 0.572 \\
\hline H119 & -2.289 & -5.948 & 1.947 \\
\hline H120 & -1.171 & -8.603 & 0.908 \\
\hline H121 & -2.786 & -8.679 & 1.662 \\
\hline H122 & -1.194 & -9.217 & 3.38 \\
\hline H123 & -1.797 & -7.554 & 3.768 \\
\hline H124 & 0.733 & -8.134 & 2.301 \\
\hline H125 & 0.917 & -8.933 & 4.571 \\
\hline H126 & 0.325 & -7.322 & 5.145 \\
\hline H127 & 2.836 & -7.634 & 3.341 \\
\hline H128 & 2.927 & -7.829 & 5.111 \\
\hline H129 & 3.573 & -5.606 & 4.879 \\
\hline H130 & 1.828 & -5.401 & 5.245 \\
\hline H131 & 2.263 & -5.697 & 2.437 \\
\hline H132 & 3.341 & -4.542 & 3.034 \\
\hline H133 & 1.47 & -3.347 & 4.087 \\
\hline H134 & 0.369 & -4.532 & 3.278 \\
\hline H135 & 2.457 & -2.861 & 1.741 \\
\hline H136 & 0.863 & -2.161 & 2.129 \\
\hline H137 & 9.814 & -5.543 & 1.388 \\
\hline H138 & 4.881 & -7.289 & -5.699 \\
\hline H139 & 0.174 & -6.63 & 2.842 \\
\hline C140 & 3.184 & -6.557 & -0.892 \\
\hline C141 & 5.502 & -5.037 & -1.345 \\
\hline C142 & 3.618 & -6.274 & -2.201 \\
\hline C143 & 3.956 & -6.079 & 0.183 \\
\hline C144 & 5.084 & -5.266 & -0.022 \\
\hline C145 & 4.778 & -5.518 & -2.448 \\
\hline C146 & 1.973 & -7.224 & -0.661 \\
\hline 0147 & 5.175 & -5.194 & -3.754 \\
\hline 0148 & 5.728 & -4.638 & 1.053 \\
\hline 0149 & 4.518 & -5.57 & -4.763 \\
\hline 0150 & 6.195 & -4.492 & -4.007 \\
\hline C151 & 5.363 & -4.784 & 2.254 \\
\hline C152 & 6.696 & -3.846 & 0.874 \\
\hline 0153 & 1.207 & -7.593 & -1.596 \\
\hline 0154 & 1.547 & -7.451 & 0.507 \\
\hline H155 & 6.372 & -4.42 & -1.522 \\
\hline H156 & 3.023 & -6.62 & -3.037 \\
\hline H157 & 3.627 & -6.276 & 1.195 \\
\hline
\end{tabular}


Table S18. Hydrogen bond interactions ( $\AA$, NH....O distance $\mathrm{d}<2.4 \AA$ ) for the lowest energy conformer of the $\left[\mathrm{H}_{9} \mathbf{L} \mathbf{2} \mathrm{A}\right]^{6+}$ adduct $(\mathrm{A}=1,2,4-\mathrm{BTC})$.

\begin{tabular}{|c|c|}
\hline N3AH....O3 & 2.14 \\
\hline N4H....O3 & 1.88 \\
\hline N3BH....O3 & 1.94 \\
\hline N3A'H....O1 & 2.01 \\
\hline N4'H....O1 & 1.97 \\
\hline N3B'H....O1 & 1.99 \\
\hline N3A'”H....O6 & 2.17 \\
\hline N4'”H....O6 & 2.06 \\
\hline N3B' 'H....O6 & 2.32 \\
\hline N3B' 'H....O5 & 2.35 \\
\hline
\end{tabular}

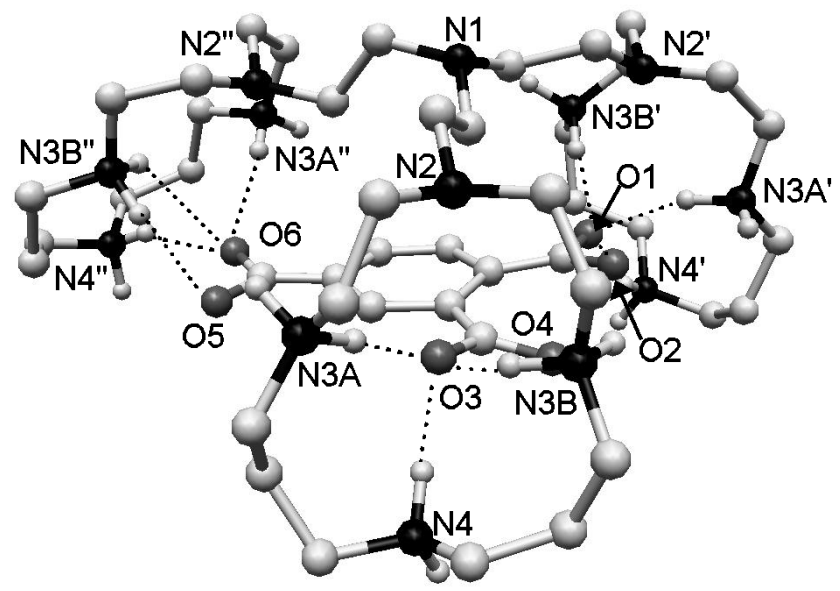

Figure S11. lowest energy conformer of the $\left[\mathrm{H}_{9} \mathrm{~L} 2 \mathrm{~A}\right]^{6+}$ adduct $(\mathrm{A}=1,2,4-\mathrm{BTC})$. 
Table S19. Cartesian coordinates for the lowest energy conformer of the complex $\left[\mathrm{H}_{9} \mathbf{L} 2 \mathrm{~A}\right]^{6+}(\mathrm{A}=$ $1,2,4-\mathrm{BTC})$

\begin{tabular}{|c|c|c|c|}
\hline atom & $x$ & $\mathrm{y}$ & $z$ \\
\hline N1 & 2.8 & -1.739 & -1.996 \\
\hline $\mathrm{C} 2$ & 4.243 & -1.759 & -1.552 \\
\hline C3 & 4.66 & -0.541 & -0.675 \\
\hline N2 & 6.13 & -0.549 & -0.335 \\
\hline C5 & 6.959 & 0.046 & -1.453 \\
\hline C6 & 8.491 & -0.225 & -1.427 \\
\hline N3A & 8.818 & -1.687 & -1.449 \\
\hline $\mathrm{C} 8$ & 10.29 & -1.957 & -1.331 \\
\hline C9 & 10.58 & -3.408 & -0.875 \\
\hline C10 & 10.481 & -3.67 & 0.649 \\
\hline N4 & 9.479 & -4.743 & 0.973 \\
\hline C12 & 9.246 & -4.941 & 2.448 \\
\hline C13 & 7.878 & -4.39 & 2.93 \\
\hline C14 & 7.809 & -2.863 & 3.189 \\
\hline N3B & 6.944 & -2.152 & 2.184 \\
\hline C16 & 7.404 & -0.752 & 1.891 \\
\hline C17 & 6.392 & 0.05 & 1.029 \\
\hline C18 & 2.534 & -2.523 & -3.255 \\
\hline C19 & 3.012 & -1.805 & -4.557 \\
\hline N2' & 3.069 & -2.707 & -5.765 \\
\hline C21 & 4.04 & -2.138 & -6.778 \\
\hline $\mathrm{C} 22$ & 4.605 & -3.118 & -7.843 \\
\hline N3A' & 5.371 & -4.248 & -7.225 \\
\hline C24 & 5.8 & -5.275 & -8.231 \\
\hline C25 & 6.451 & -6.511 & -7.559 \\
\hline $\mathrm{C} 26$ & 5.703 & -7.857 & -7.743 \\
\hline N4' & 4.615 & -8.066 & -6.725 \\
\hline C28 & 3.223 & -7.87 & -7.259 \\
\hline C29 & 2.17 & -7.903 & -6.124 \\
\hline $\mathrm{C} 30$ & 1.052 & -6.835 & -6.209 \\
\hline $\mathrm{N}^{\prime} \mathrm{B}^{\prime}$ & 1.51 & -5.469 & -5.766 \\
\hline C32 & 1.492 & -4.44 & -6.861 \\
\hline C33 & 1.693 & -2.995 & -6.33 \\
\hline C34 & 1.783 & -2 & -0.908 \\
\hline C35 & 1.847 & -3.41 & -0.221 \\
\hline $\mathrm{N} 2^{\prime \prime}$ & 0.778 & -3.76 & 0.78 \\
\hline C37 & -0.608 & -3.965 & 0.221 \\
\hline C38 & -0.774 & -5.211 & -0.698 \\
\hline N3A ${ }^{\prime \prime}$ & -0.395 & -6.5 & -0.021 \\
\hline C40 & -1.557 & -7.278 & 0.528 \\
\hline C41 & -1.093 & -8.389 & 1.5 \\
\hline C42 & -0.948 & -7.955 & 2.98 \\
\hline N4' & 0.325 & -8.467 & 3.594 \\
\hline C44 & 0.529 & -8.04 & 5.022 \\
\hline C45 & 1.534 & -6.868 & 5.174 \\
\hline C46 & 0.948 & -5.443 & 5.005 \\
\hline N3B' ${ }^{\prime}$ & 1.202 & -4.889 & 3.634 \\
\hline C48 & 0.37 & -3.68 & 3.328 \\
\hline C49 & 0.844 & -2.944 & 2.044 \\
\hline H5O & 4.449 & -2.688 & -0.98 \\
\hline H51 & 4.915 & -1.774 & -2.438 \\
\hline H52 & 4.047 & -0.616 & 0.253 \\
\hline
\end{tabular}




\begin{tabular}{|c|c|c|c|}
\hline H53 & 4.372 & 0.407 & -1.181 \\
\hline H5 4 & 6.807 & 1.149 & -1.477 \\
\hline H55 & 6.6 & -0.344 & -2.435 \\
\hline H56 & 8.939 & 0.226 & -0.519 \\
\hline H57 & 8.953 & 0.237 & -2.326 \\
\hline H58 & 8.47 & -2.1 & -2.338 \\
\hline H59 & 8.33 & -2.15 & -0.656 \\
\hline $\mathrm{H} 60$ & 10.739 & -1.235 & -0.618 \\
\hline $\mathrm{H} 61$ & 10.733 & -1.792 & -2.336 \\
\hline H62 & 11.622 & -3.65 & -1.188 \\
\hline H63 & 9.917 & -4.102 & -1.435 \\
\hline H6 4 & 10.187 & -2.752 & 1.198 \\
\hline $\mathrm{H} 65$ & 11.467 & -4.012 & 1.028 \\
\hline H66 & 8.573 & -4.492 & 0.528 \\
\hline $\mathrm{H} 67$ & 10.082 & -4.501 & 3.027 \\
\hline H68 & 9.26 & -6.04 & 2.617 \\
\hline H69 & 7.643 & -4.898 & 3.894 \\
\hline H70 & 7.088 & -4.702 & 2.214 \\
\hline H71 & 8.824 & -2.415 & 3.188 \\
\hline H72 & 7.352 & -2.682 & 4.185 \\
\hline H73 & 5.972 & -2.113 & 2.551 \\
\hline H74 & 6.947 & -2.696 & 1.298 \\
\hline H75 & 8.387 & -0.817 & 1.384 \\
\hline H76 & 7.534 & -0.225 & 2.861 \\
\hline H77 & 6.763 & 1.095 & 0.931 \\
\hline H78 & 5.456 & 0.113 & 1.635 \\
\hline H79 & 3.004 & -3.526 & -3.184 \\
\hline $\mathrm{H} 80$ & 1.437 & -2.684 & -3.375 \\
\hline H81 & 2.351 & -0.931 & -4.753 \\
\hline H82 & 4.027 & -1.401 & -4.339 \\
\hline H83 & 4.937 & -1.727 & -6.254 \\
\hline H84 & 3.567 & -1.281 & -7.31 \\
\hline H85 & 5.305 & -2.563 & -8.503 \\
\hline H86 & 3.786 & -3.532 & -8.463 \\
\hline H87 & 4.778 & -4.707 & -6.504 \\
\hline H8 8 & 6.218 & -3.862 & -6.761 \\
\hline H89 & 6.542 & -4.792 & -8.902 \\
\hline H9O & 4.912 & -5.563 & -8.832 \\
\hline H91 & 6.616 & -6.329 & -6.473 \\
\hline H92 & 7.463 & -6.634 & -8.01 \\
\hline H93 & 6.428 & -8.686 & -7.595 \\
\hline H94 & 5.288 & -7.953 & -8.767 \\
\hline H95 & 4.692 & -9.032 & -6.349 \\
\hline H96 & 3.024 & -8.689 & -7.982 \\
\hline H97 & 3.201 & -6.901 & -7.798 \\
\hline H98 & 2.664 & -7.814 & -5.133 \\
\hline H99 & 1.681 & -8.904 & -6.146 \\
\hline H100 & 0.229 & -7.124 & -5.521 \\
\hline H101 & 0.634 & -6.787 & -7.235 \\
\hline H102 & 2.483 & -5.549 & -5.404 \\
\hline H103 & 0.891 & -5.144 & -4.997 \\
\hline H10 4 & 0.501 & -4.49 & -7.36 \\
\hline H105 & 2.275 & -4.714 & -7.593 \\
\hline H106 & 0.904 & -2.843 & -5.557 \\
\hline H107 & 1.454 & -2.28 & -7.151 \\
\hline H108 & 1.901 & -1.23 & -0.115 \\
\hline H109 & 0.763 & -1.859 & -1.328 \\
\hline H110 & 1.857 & -4.18 & -1.022 \\
\hline H111 & 2.833 & -3.486 & 0.289 \\
\hline H112 & -0.922 & -3.061 & -0.345 \\
\hline
\end{tabular}




\begin{tabular}{|c|c|c|c|}
\hline H113 & -1.338 & -4.089 & 1.054 \\
\hline H114 & -0.116 & -5.112 & -1.585 \\
\hline H115 & -1.821 & -5.277 & -1.058 \\
\hline H116 & 0.26 & -6.285 & 0.758 \\
\hline H117 & 0.1 & -7.098 & -0.712 \\
\hline H118 & -2.082 & -7.736 & -0.337 \\
\hline H119 & -2.249 & -6.574 & 1.035 \\
\hline H120 & -0.139 & -8.822 & 1.125 \\
\hline H121 & -1.849 & -9.208 & 1.459 \\
\hline H122 & -1.794 & -8.367 & 3.571 \\
\hline H123 & -0.946 & -6.85 & 3.077 \\
\hline H124 & 0.314 & -9.505 & 3.558 \\
\hline H125 & 0.943 & -8.928 & 5.548 \\
\hline H126 & -0.448 & -7.802 & 5.488 \\
\hline H127 & 2.389 & -7.02 & 4.478 \\
\hline H128 & 1.957 & -6.93 & 6.204 \\
\hline H129 & 1.437 & -4.757 & 5.729 \\
\hline H130 & -0.146 & -5.44 & 5.195 \\
\hline H131 & 0.978 & -5.627 & 2.936 \\
\hline H132 & 2.208 & -4.642 & 3.549 \\
\hline H133 & 0.446 & -2.978 & 4.185 \\
\hline H134 & -0.684 & -4.008 & 3.22 \\
\hline H135 & 1.894 & -2.621 & 2.238 \\
\hline H136 & 0.241 & -2.017 & 1.923 \\
\hline H137 & 9.813 & -5.64 & 0.566 \\
\hline H138 & 4.761 & -7.391 & -5.947 \\
\hline H139 & 1.125 & -8.118 & 3.03 \\
\hline C140 & 4.734 & -5.62 & -2.532 \\
\hline C141 & 3.734 & -6.258 & 0.077 \\
\hline C142 & 5.446 & -5.183 & -1.371 \\
\hline C143 & 3.593 & -6.443 & -2.333 \\
\hline C144 & 3.107 & -6.755 & -1.069 \\
\hline C145 & 4.9 & -5.499 & -0.097 \\
\hline C146 & 3.033 & -6.819 & -3.181 \\
\hline 0147 & 2.212 & -7.361 & -0.988 \\
\hline 0148 & 5.394 & -5.133 & 0.796 \\
\hline 0149 & 5.025 & -5.303 & -3.884 \\
\hline 0150 & 6.624 & -4.395 & -1.339 \\
\hline C151 & 3.194 & -6.472 & 1.355 \\
\hline C152 & 4.38 & -5.794 & -4.855 \\
\hline 0153 & 5.87 & -4.469 & -4.298 \\
\hline 0154 & 7.026 & -3.814 & -0.289 \\
\hline H155 & 7.423 & -4.208 & -2.291 \\
\hline H156 & 3.731 & -6.012 & 2.404 \\
\hline H157 & 2.111 & -7.097 & 1.536 \\
\hline
\end{tabular}


Table S20. Hydrogen bond interactions ( $\AA$, NH....O distance $d<2.4 \AA$ ) for the lowest energy conformer of the $\left[\mathrm{H}_{9} \mathbf{L} \mathbf{2} \mathrm{A}\right]^{6+}$ adduct $(\mathrm{A}=1,2,3-\mathrm{BTC})$.

$\begin{array}{ll}\text { N3AH.... } & 1.94 \\ \text { N4H....O3 } & 1.97 \\ \text { N3BH....O3 } & 1.97 \\ \text { N3A'H....O1 } & 2.02 \\ \text { N3A'H....O6 } & 2.16 \\ \text { N4'H....O1 } & 1.88 \\ \text { N3B'H....O1 } & 2.25 \\ \text { N3B'H....O2 } & 2.27\end{array}$

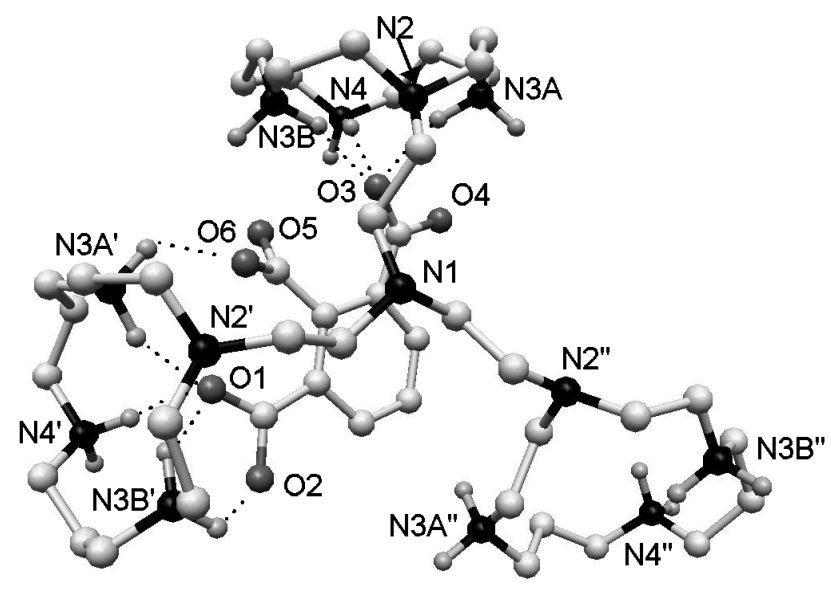

Figure S12. Lowest energy conformer of the $\left[\mathrm{H}_{9} \mathbf{L} 2 \mathrm{~A}\right]^{6+}$ adduct $(\mathrm{A}=1,2,3-\mathrm{BTC})$. 
Table S21. Cartesian coordinates of the lowest energy conformer of the complex $\left[\mathrm{H}_{9} \mathbf{L} \mathbf{2} \mathrm{A}\right]^{6+}(\mathrm{A}=$ 1,2,3-BTC)

\begin{tabular}{|c|c|c|c|}
\hline atom & $\mathrm{x}$ & $\mathrm{y}$ & $\mathrm{z}$ \\
\hline N1 & -0.556 & -6.497 & -2.435 \\
\hline $\mathrm{C} 2$ & -0.227 & -5.785 & -1.148 \\
\hline C3 & -1.074 & -6.228 & 0.083 \\
\hline $\mathrm{N} 2$ & -0.321 & -6.078 & 1.385 \\
\hline C5 & -0.674 & -7.193 & 2.339 \\
\hline $\mathrm{C} 6$ & 0.382 & -7.487 & 3.442 \\
\hline N3A & 1.654 & -8.054 & 2.878 \\
\hline $\mathrm{C} 8$ & 2.661 & -8.46 & 3.923 \\
\hline C9 & 3.505 & -7.288 & 4.491 \\
\hline C10 & 4.84 & -6.967 & 3.764 \\
\hline N4 & 4.75 & -5.812 & 2.8 \\
\hline C12 & 4.821 & -4.454 & 3.446 \\
\hline C13 & 4.081 & -3.369 & 2.628 \\
\hline C14 & 2.533 & -3.374 & 2.719 \\
\hline N3B & 1.877 & -3.817 & 1.439 \\
\hline C16 & 0.393 & -3.564 & 1.4 \\
\hline C17 & -0.467 & -4.709 & 2.017 \\
\hline C18 & -0.171 & -5.668 & -3.637 \\
\hline C19 & -1.186 & -4.537 & -3.981 \\
\hline $\mathrm{N}^{\prime}{ }^{\prime}$ & -0.469 & -3.287 & -4.437 \\
\hline $\mathrm{C} 21$ & -0.583 & -2.166 & -3.435 \\
\hline $\mathrm{C} 22$ & 0.51 & -1.063 & -3.527 \\
\hline $\mathrm{N}^{\prime} \mathrm{A}^{\prime}$ & 1.872 & -1.586 & -3.189 \\
\hline C24 & 2.929 & -0.521 & -3.186 \\
\hline C25 & 4.356 & -1.126 & -3.204 \\
\hline C26 & 5.146 & -0.973 & -4.53 \\
\hline $\mathrm{N} 4^{\prime}$ & 4.728 & -1.955 & -5.593 \\
\hline $\mathrm{C} 28$ & 3.896 & -1.349 & -6.689 \\
\hline C29 & 3.473 & -2.396 & -7.75 \\
\hline C30 & 1.95 & -2.615 & -7.915 \\
\hline $\mathrm{N}^{\prime} \mathrm{B}^{\prime}$ & 1.411 & -3.588 & -6.908 \\
\hline C32 & -0.084 & -3.744 & -6.935 \\
\hline C33 & -0.798 & -2.885 & -5.854 \\
\hline C34 & 0.068 & -7.869 & -2.481 \\
\hline C35 & -0.694 & -8.858 & -3.416 \\
\hline N2' ${ }^{\prime}$ & -0.123 & -10.251 & -3.422 \\
\hline C37 & 1.26 & -10.401 & -4.014 \\
\hline C38 & 1.418 & -10.074 & -5.529 \\
\hline N3A $A^{\prime \prime}$ & 2.855 & -10.108 & -5.976 \\
\hline C40 & 3.354 & -11.455 & -6.431 \\
\hline C41 & 4.34 & -12.081 & -5.416 \\
\hline $\mathrm{C} 42$ & 4.569 & -13.606 & -5.578 \\
\hline $\mathrm{N} 4^{\prime \prime}$ & 3.712 & -14.427 & -4.652 \\
\hline C44 & 2.452 & -14.954 & -5.282 \\
\hline C45 & 1.465 & -15.539 & -4.239 \\
\hline C4 6 & 0.763 & -14.528 & -3.298 \\
\hline N3B' & -0.236 & -13.662 & -4.013 \\
\hline C48 & -0.915 & -12.664 & -3.124 \\
\hline C49 & -1.12 & -11.301 & -3.84 \\
\hline H5O & 0.855 & -5.9 & -0.918 \\
\hline H51 & -0.418 & -4.694 & -1.263 \\
\hline H52 & -1.363 & -7.291 & -0.088 \\
\hline H53 & -2.022 & -5.646 & 0.102 \\
\hline
\end{tabular}




\begin{tabular}{|c|c|c|c|}
\hline H5 4 & -0.806 & -8.151 & 1.782 \\
\hline H55 & -1.65 & -6.976 & 2.831 \\
\hline H56 & -0.039 & -8.233 & 4.148 \\
\hline H57 & 0.633 & -6.563 & 3.999 \\
\hline H58 & 2.088 & -7.352 & 2.245 \\
\hline H59 & 1.412 & -8.897 & 2.318 \\
\hline H60 & 3.321 & -9.215 & 3.445 \\
\hline H61 & 2.119 & -8.968 & 4.748 \\
\hline H62 & 3.765 & -7.556 & 5.541 \\
\hline H63 & 2.878 & -6.373 & 4.548 \\
\hline H64 & 5.185 & -7.841 & 3.173 \\
\hline H65 & 5.63 & -6.734 & 4.508 \\
\hline H66 & 5.541 & -5.89 & 2.129 \\
\hline H67 & 5.897 & -4.188 & 3.52 \\
\hline H68 & 4.399 & -4.508 & 4.47 \\
\hline H69 & 4.418 & -3.408 & 1.57 \\
\hline H70 & 4.423 & -2.383 & 3.021 \\
\hline H71 & 2.192 & -2.338 & 2.934 \\
\hline H72 & 2.171 & -4.041 & 3.528 \\
\hline H73 & 2.043 & -4.834 & 1.303 \\
\hline H74 & 2.318 & -3.302 & 0.65 \\
\hline H75 & 0.124 & -3.414 & 0.336 \\
\hline H76 & 0.178 & -2.614 & 1.93 \\
\hline H77 & -1.536 & -4.396 & 1.994 \\
\hline H78 & -0.179 & -4.749 & 3.093 \\
\hline H79 & 0.846 & -5.245 & -3.492 \\
\hline $\mathrm{H} 80$ & -0.118 & -6.307 & -4.548 \\
\hline H81 & -1.86 & -4.934 & -4.776 \\
\hline H82 & -1.841 & -4.336 & -3.103 \\
\hline H83 & -0.524 & -2.58 & -2.4 \\
\hline H84 & -1.582 & -1.682 & -3.529 \\
\hline H85 & 0.265 & -0.258 & -2.802 \\
\hline H86 & 0.547 & -0.632 & -4.547 \\
\hline H87 & 2.134 & -2.319 & -3.878 \\
\hline H8 8 & 1.838 & -2.021 & -2.246 \\
\hline H89 & 2.793 & 0.069 & -2.254 \\
\hline H9O & 2.766 & 0.147 & -4.056 \\
\hline H91 & 4.334 & -2.2 & -2.912 \\
\hline H92 & 4.943 & -0.603 & -2.413 \\
\hline H93 & 6.219 & -1.18 & -4.325 \\
\hline H94 & 5.073 & 0.062 & -4.92 \\
\hline H95 & 5.582 & -2.368 & -6.017 \\
\hline H96 & 4.511 & -0.564 & -7.178 \\
\hline H97 & 3.013 & -0.869 & -6.219 \\
\hline H98 & 3.961 & -3.375 & -7.549 \\
\hline H99 & 3.864 & -2.048 & -8.734 \\
\hline $\mathrm{H} 100$ & 1.748 & -3.037 & -8.923 \\
\hline $\mathrm{H} 101$ & 1.398 & -1.658 & -7.809 \\
\hline $\mathrm{H} 102$ & 1.689 & -3.268 & -5.958 \\
\hline $\mathrm{H} 103$ & 1.846 & -4.517 & -7.081 \\
\hline $\mathrm{H} 104$ & -0.295 & -4.82 & -6.761 \\
\hline $\mathrm{H} 105$ & -0.466 & -3.48 & -7.942 \\
\hline $\mathrm{H} 106$ & -1.897 & -2.94 & -6.02 \\
\hline $\mathrm{H107}$ & -0.504 & -1.827 & -6.041 \\
\hline $\mathrm{H1O} 8$ & 1.134 & -7.796 & -2.792 \\
\hline H109 & 0.059 & -8.337 & -1.469 \\
\hline H110 & -1.745 & -8.884 & -3.043 \\
\hline H111 & -0.723 & -8.455 & -4.451 \\
\hline $\mathrm{H} 112$ & 1.617 & -11.44 & -3.841 \\
\hline $\mathrm{H} 113$ & 1.956 & -9.745 & -3.447 \\
\hline
\end{tabular}




\begin{tabular}{|c|c|c|c|}
\hline H114 & 1.06 & -9.042 & -5.724 \\
\hline H115 & 0.826 & -10.771 & -6.153 \\
\hline H116 & 3.449 & -9.787 & -5.185 \\
\hline H117 & 2.963 & -9.439 & -6.765 \\
\hline H118 & 3.878 & -11.286 & -7.397 \\
\hline H119 & 2.491 & $-12 \cdot 122$ & -6.622 \\
\hline $\mathrm{H} 12 \mathrm{O}$ & 3.996 & -11.869 & -4.38 \\
\hline H121 & 5.326 & -11.574 & -5.537 \\
\hline $\mathrm{H} 122$ & 5.622 & -13.837 & -5.303 \\
\hline H123 & 4.416 & -13.944 & -6.622 \\
\hline H124 & 4.272 & -15.235 & -4.313 \\
\hline H125 & 2.751 & -15.753 & -5.993 \\
\hline H126 & 1.968 & -14.134 & -5.849 \\
\hline H127 & 2.015 & -16.271 & -3.604 \\
\hline H128 & 0.686 & -16.121 & -4.784 \\
\hline H129 & 1.508 & -13.866 & -2.811 \\
\hline H130 & 0.213 & -15.089 & -2.511 \\
\hline H131 & -0.961 & -14.276 & -4.436 \\
\hline H132 & 0.254 & -13.148 & -4.773 \\
\hline H133 & -0.333 & -12.525 & -2.19 \\
\hline H134 & -1.905 & -13.093 & -2.859 \\
\hline H135 & -2.152 & -10.975 & -3.566 \\
\hline H136 & -1.112 & -11.426 & -4.945 \\
\hline H137 & 3.862 & -5.89 & 2.267 \\
\hline H138 & 4.178 & -2.715 & -5.142 \\
\hline H139 & 3.448 & -13.835 & -3.84 \\
\hline C140 & 4.472 & -8.533 & -3.332 \\
\hline C141 & 4.103 & -7.472 & -4.139 \\
\hline C142 & 4.345 & -8.422 & -1.96 \\
\hline C143 & 3.858 & -7.245 & -1.355 \\
\hline C144 & 3.662 & -6.233 & -3.613 \\
\hline C145 & 3.416 & -5.226 & -4.585 \\
\hline C146 & 3.553 & -7.344 & 0.029 \\
\hline 0147 & 3.079 & -4.034 & -4.366 \\
\hline 0148 & 3.511 & -5.436 & -5.83 \\
\hline 0149 & 2.866 & -6.508 & 0.68 \\
\hline 0150 & 3.901 & -8.329 & 0.742 \\
\hline C151 & 3.593 & -6.097 & -2.182 \\
\hline C152 & 3.379 & -4.849 & -1.559 \\
\hline 0153 & 4.116 & -4.457 & -0.61 \\
\hline 0154 & 2.451 & -4.047 & -1.846 \\
\hline H155 & 4.572 & -9.298 & -1.361 \\
\hline H156 & 4.83 & -9.457 & -3.772 \\
\hline H157 & 4.21 & -7.607 & -5.21 \\
\hline
\end{tabular}


Table S22. Thermodynamics parameters for $\mathbf{L} 1$ and $\mathbf{L} 2$ protonation $\left(\mathrm{NMe}_{4} \mathrm{Cl} 0.1 \mathrm{M}, 298.1 \mathrm{~K}\right)$

$\begin{array}{crc}\Delta \mathrm{H}^{\circ} & \mathrm{T} \Delta \mathrm{S}^{\circ} & \Delta \mathrm{G}^{\circ} \\ \left(\mathrm{KJ} \mathrm{mol}^{-1}\right) & \left(\mathrm{KJ} \mathrm{mol}^{-1}\right) & \left(\mathrm{KJ} \mathrm{mol}^{-1}\right)\end{array}$

$\mathrm{L}+\mathrm{H}^{+}=\mathrm{HL}^{+}$

$\mathrm{HL}^{+}+\mathrm{H}^{+}=\mathrm{H}_{2} \mathrm{~L}^{2+}$

$\mathrm{HL}_{2}^{2+}+\mathrm{H}^{+}=\mathrm{H}_{3} \mathrm{~L}^{3+}$

$\mathrm{HL}_{3}^{3+}+\mathrm{H}^{+}=\mathrm{H}_{4} \mathrm{~L}^{4+}$

$\mathrm{HL}_{4}{ }^{4+}+\mathrm{H}^{+}=\mathrm{H}_{5} \mathrm{~L}^{5+}$

$\mathrm{HL}_{5}{ }^{5+}+\mathrm{H}^{+}=\mathrm{H}_{6} \mathrm{~L}^{6+}$

$\mathrm{HL}_{6}{ }^{6+}+\mathrm{H}^{+}=\mathrm{H}_{7} \mathrm{~L}^{7+}$

$\mathrm{HL}_{7}{ }^{7+}+\mathrm{H}^{+}=\mathrm{H}_{8} \mathrm{~L}^{8+}$

$\mathrm{HL}_{8}^{8+}+\mathrm{H}^{+}=\mathrm{H}_{9} \mathrm{~L}^{9+}$

$\mathrm{HL}_{9}{ }^{9+}+\mathrm{H}^{+}=\mathrm{H}_{10} \mathrm{~L}^{10+}$

$\mathrm{HL}_{10}{ }^{10+}+\mathrm{H}^{+}=\mathrm{H}_{11} \mathrm{~L}^{11+}$
L1

$\begin{array}{lll}-34.4(2) & 23.2 & -57.6(2) \\ -46.8(2) & 6.6(2) & -53.4(2) \\ -39.0(2) & 12.0(2) & -51.0(2) \\ -51.9(1) & -4.2(1) & -47.7(1) \\ -47.6(2) & -2.6(2) & -45.0(2) \\ -49.1(1) & -5.3(1) & -43.8(1) \\ -34.9(2) & 1.0(2) & -35.9(2) \\ -4.7(1) & 18.7(1) & -23.4(1)\end{array}$

$-34.4(2)$

$3.4(1)$
L2

$\begin{array}{clc}\begin{array}{c}\Delta \mathrm{H}^{\circ} \\ \left(\mathrm{KJ} \mathrm{mol}^{-1}\right)\end{array} & \begin{array}{c}\mathrm{T} \Delta \mathrm{S}^{\circ} \\ \left(\mathrm{KJ} \mathrm{mol}^{-1}\right)\end{array} & \begin{array}{c}\Delta \mathrm{G}^{\circ} \\ \left(\mathrm{KJ} \mathrm{mol}^{-1}\right)\end{array} \\ -41.8(2) & 19.5(2) & -61.3(2) \\ -45.2(2) & 13.8(2) & -59.0(2) \\ -38.5(1) & 16.1(1) & -54.6(1) \\ -47.3(2) & 7.0(2) & -54.3(2) \\ -44.8(3) & 2.6(3) & -47.4(3) \\ -46.9(2) & -0.1(2) & -46.8(2) \\ -48.5(3) & -8.2(3) & -40.3(3) \\ -41.4(2) & -2.9(2) & -38.5(2) \\ -45.2(2) & -12.7(2) & -32.5(2) \\ -35.9(2) & -7.4(2) & -28.5(2) \\ -11.7(1) & 11.7(1) & -23.4(1)\end{array}$

Table S23. Thermodynamics parameters for protonation of the BTC acids $\left(\mathrm{NMe}_{4} \mathrm{Cl} 0.1 \mathrm{M}, 298.1\right.$ $\mathrm{K})$

$\log \mathrm{K}$

$\begin{array}{lccc} & 1,3,5-\mathrm{BTC} & 1,2,3-\mathrm{BTC} & 1,2,4-\mathrm{BTC} \\ \mathrm{A}^{3-}+\mathrm{H}^{+}=\mathrm{HA}^{2-} & 4.64 & 5.49 & 5.00 \\ \mathrm{HA}^{2-}+\mathrm{H}^{+}=\mathrm{H}_{2} \mathrm{~A}^{-} & 3.65 & 3.99 & 3.67 \\ \mathrm{H}_{2} \mathrm{~A}^{2-}+\mathrm{H}^{+}=\mathrm{H}_{3} \mathrm{~A} & 3.10 & 2.66 & 2.41\end{array}$

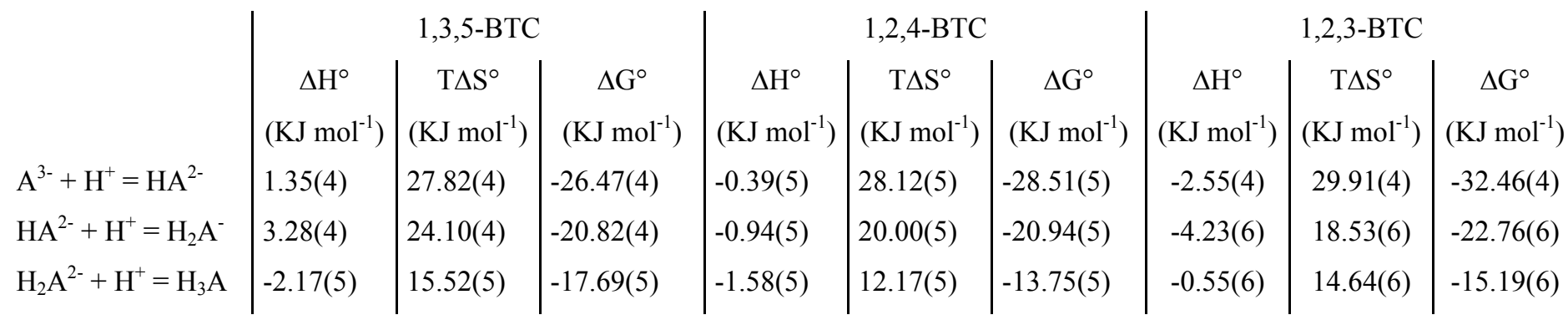




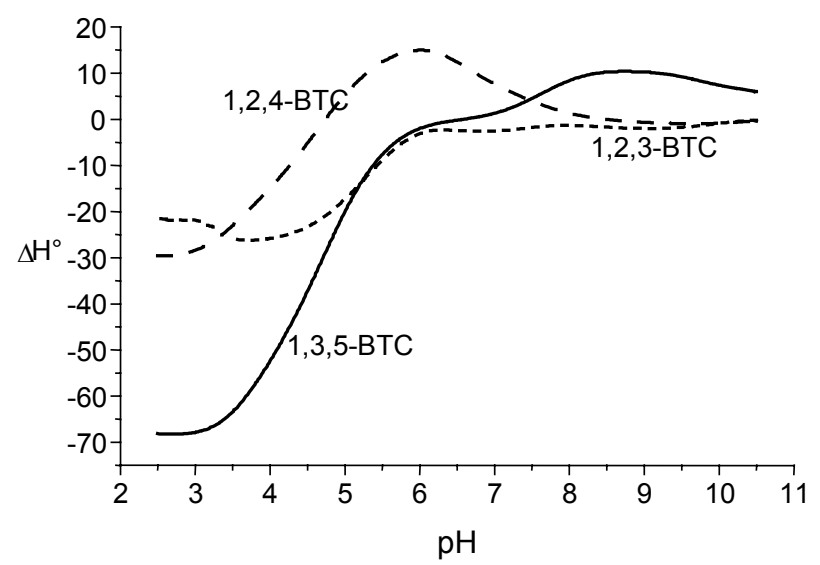

a

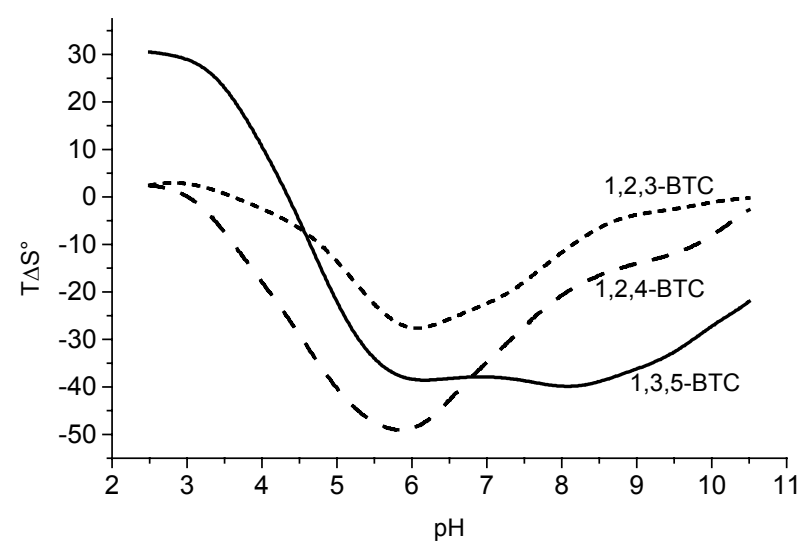

b

Figure S13. $\mathrm{pH}$ dependence of the enthalpic (a) and entropic (b) conditional contributions for the complexation of 1,3,5-BTC (solid lines), 1,2,4-BTC (dashed lines) and 1,2,3-BTC (dotted lines) with $\mathbf{L 2}$ 